\title{
Pseudo-differential Calculus on Manifolds with Geometric Singularities
}

\author{
B.-W. Schulze
}

\begin{abstract}
Differential and pseudo-differential operators on a manifold with (regular) geometric singularities can be studied within a calculus, inspired by the concept of classical pseudo-differential operators on a $C^{\infty}$ manifold. In the singular case the operators form an algebra with a principal symbolic hierarchy $\sigma=\left(\sigma_{j}\right)_{0 \leq j \leq k}$, with $k$ being the order of the singularity and $\sigma_{k}$ operator-valued for $k \geq 1$. The symbols determine ellipticity and the nature of parametrices. It is typical in this theory that, similarly as in boundary value problems (which are special edge problems, where the edge is just the boundary), there are trace, potential and Green operators, associated with the various strata of the configuration. The operators, obtained from the symbols by various quantisations, act in weighted distribution spaces with multiple weights. We outline some essential elements of this calculus, give examples and also comment on new challenges and interesting problems of the recent development.
\end{abstract}

\section{Contents}

$\begin{array}{lr}\text { Introduction } & 2\end{array}$

1 Differential operators on manifolds with higher singularities $\mathbf{5}$

1.1 Conical singularities . . . . . . . . . . . . . . 5 5

1.2 Boundaries and edges . . . . . . . . . . . . . . . 13

1.3 Higher singularities . . . . . . . . . . . . . . . . . 20

2 Pseudo-differential operators with parameters and conical exits to infinity $\quad 26$

2.1 Classical operators with parameters . . . . . . . . . . . 26

2.2 Corner-degenerate operators . . . . . . . . . . . . . . . . 29

2.3 Symbols with twisted homogeneity . . . . . . . . . . . 31 
2.4 Conical exits to infinity . . . . . . . . . . . . . . . . 32

3 A hierarchy of operator algebras $\quad 36$

3.1 The program of the iterative calculus . . . . . . . . . . 36

3.2 Ellipticity and an analogue of the Atiyah-Bott obstruction . . 43

3.3 Weighted spaces . . . . . . . . . . . . . . . . . . . . . . . . . . . . . 51

3.4 Concluding remarks . . . . . . . . . . . . . . . . 51

$\begin{array}{ll}\text { References } & 53\end{array}$

\section{Introduction}

The analysis on manifolds with singularities (certain stratified spaces in our terminology, for instance, with conical, edge, or corner singularities) is motivated by models of physics or the applied sciences, and also by structures of geometry and topology.

These lectures give an introduction and a survey on some new developments in this field. We consider ellipticity of operators referring to a principal symbolic hierarchy, with components contributed by the various strata of the configuration $M$. For instance, if $M$ is an open $C^{\infty}$ manifold, then the ellipticity of a (classical pseudo-)differential operator $A$ is the standard one, namely, non-vanishing of the homogeneous principal symbol $\sigma_{\psi}(A)$, given on $T^{*} M \backslash 0$ (the cotangent bundle of $M$ minus the zero-section). If $M$ is a $C^{\infty}$ manifold with boundary, then $M$ is the disjoint union of two strata, namely, $Y^{0}:=\operatorname{int} M$ and $Y^{1}:=\partial M$, and we have the interior symbol $\sigma_{\psi}(A)$ associated with $Y^{0}$ and the boundary symbol $\sigma_{\partial}(A)$ associated with $Y^{1}$. The latter one is operator-valued and a kind of semi-quantised object containing $\sigma_{\psi}(A)$, frozen at the boundary. At the same time, ellipticity requires boundary conditions, formulated as additional entries in a block matrix of boundary symbols, with $\sigma_{\partial}(A)$ in the upper left corner, and other entries representing trace conditions (for instance, Dirichlet) and potential data (for instance, double layer potentials, occurring in parametrices). In addition, compositions and parametrix constructions generate Green symbols in the upper left corners; those constitute (together with an 'interior parametrix') what is known in classical cases as Green's function of a boundary value problem.

If $M$ has conical singularities $Y^{1}:=\left\{v_{1}, \ldots, v_{N}\right\} \subset M$ or smooth edges $Y^{1} \subset M$ of any codimension, then $M \backslash Y^{1}=: Y^{0}$ is smooth, and again, from the disjoint union $M=Y^{0} \cup Y^{1}$ of strata, we expect two principal symbolic components, in this case $\sigma_{\psi}(A)$ on $Y^{0}$ as before and $\sigma_{\mathrm{c}}(A)$ at $Y^{1}$ in 
the conical and $\sigma_{\wedge}(A)$ in the edge case, called the (principal) conormal and edge symbol, respectively (see the formulas (10) and (22) below). The latter ones are again operator-valued.

In higher singularities of order $k \in \mathbb{N}$ the space $M$ is a disjoint union of $C^{\infty}$ manifolds

$$
M=\bigcup_{j=0}^{k} Y^{j},
$$

where (of course) some extra conditions specify the way of how they are connected with each other, and operators $A$ on $M$ have a principal symbolic hierarchy

$$
\sigma(A)=\left(\sigma_{j}(A)\right)_{j=0, \ldots, k},
$$

where $\sigma_{0}(A):=\sigma_{\psi}(A), \sigma_{1}(A):=\sigma_{\wedge}(A)$ (or $=\sigma_{\mathrm{c}}(A)$ ), etc. In the edge case which is a generalisation of the case with boundary, there are again block matrix edge symbols belonging to operators of trace and potential type, plus Green (and Mellin) symbols in the upper left corner. On the level of operators they contribute an adequate extra information from the edge. $A$ similar block matrix structure with additional trace, potential and Green ( + Mellin) data along the lower-dimensional strata is to be expected for higher singularities. This is a very satisfying concept, iteratively organised, which admits to understand a large variety of concrete problems from the above-mentioned areas, and with new beautiful and challenging problems. Nevertheless, as we shall see, in the elliptic case the effect of such data of trace and potential type can often be formulated in terms of extra operators (smoothing over $Y$ but not compact) in the upper left corner, without loss of information from the point of view of the index. It is important to specify the nature of operators that are recognised as the 'natural ones' on a manifold $M$ with singularities. For instance, if $M$ has a boundary $(\neq \emptyset)$, then a problem is to distinguish operators with or without the transmission property (the latter is a subcase of the edge calculus). In geometric singularities, e.g., when we consider a wedge

$$
X^{\Delta} \times \Omega
$$

where $X^{\Delta}:=\left(\overline{\mathbb{R}}_{+} \times X\right) /(\{0\} \times X)$ is a cone with $C^{\infty}$ base $X$ and $\Omega \subseteq \mathbb{R}^{q}$ open, then the Laplace-Beltrami operator belonging to a wedge metric on $\mathbb{R}_{+} \times X \times \Omega \ni(r, x, y)$

$$
d r^{2}+r^{2} g_{X}(r, y)+d y^{2}
$$

where $g_{X}(r, y)$ is family of Riemannian metrics on $X$, smoothly depending on $(r, y)$ up to $r=0$, should belong to the calculus. Such operators (for any 
order $\mu \in \mathbb{N}$, not necessarily of second order) have the form

$$
A=r^{-\mu} \sum_{j+|\alpha| \leq \mu} a_{j \alpha}(r, y)\left(-r \frac{\partial}{\partial r}\right)^{j}\left(r D_{y}\right)^{\alpha}
$$

with coefficients $a_{j \alpha} \in C^{\infty}\left(\overline{\mathbb{R}}_{+} \times \Omega\right.$, $\left.\operatorname{Diff}^{\mu-(j+|\alpha|)}(X)\right)$ (here $\operatorname{Diff}^{\nu}(\cdot)$ denotes the space of all differential operators of order $\nu$ on the smooth manifold in parentheses). Operators of the form (3) are degenerate at $r=0$ in a typical way, and we call them edge-degenerate. In the case $q=\operatorname{dim} \Omega=0$ the $y$ variable does not occur; the corresponding operators on the open stretched cone $X^{\wedge}:=\mathbb{R}_{+} \times X$ will be called of Fuchs type.

On manifolds $M$ with higher singularities we also have a specific choice of typical differential operators $A$ which are corner degenerate in stretched coordinates, with symbols (2), ellipticity, and the construction of parametrices. Those are expected to belong to corresponding pseudo-differential operator algebras with similar symbolic hierarchies. Here we outline some elements of such a calculus, where the iterative structure of the approach is one of the essential points. We assume that things are done up to the singularity order $k-1, k \geq 2$, and then pass to the case $k$.

What concerns conical singularities and smooth edges, i.e., the case $k=1$, the material may be found in [71], [72], [75], [16], [19], [20], [90], [13], [10], [42], [85]. The calculus for corner singularities of order $k=2$ is studied under different aspects in [71], [70], [77], [73], [88], [78], [80], [40], [88], [39], [45], [7], [8], [27], see also the monograph [28] (in preparation), moreover, [12] and [47]. The theory is voluminous, already for case $k=1$, and by no means complete (for instance, in respect to an analogue of the index theory). The structures for higher singularities are much more involved and subject of current research. In the present paper we content ourselves with describing the main ideas of our calculus. Many other authors studied operators on manifolds with singularities from different point of views. Ideas, approaches and priorities are contributed by wide fields of applied mathematics, analysis of partial differential operators, mathematical physics, index theory, geometry and topology. Let us give a few references here, in particular, [92], [93], [51], [53], [54], [55], [60], [52], [50], [52], [57], [58], [59], [56], [3]. A more complete survey on aspects of the recent development and also of historical background and sources of ideas is given in [80]. 


\section{Differential operators on manifolds with higher singularities}

\subsection{Conical singularities}

Definition 1.1 A manifold $M$ with conical singularity $\{v\}$ is a topological space with a chosen point $v \in M$ such that

(i) $M \backslash\{v\}$ is a $C^{\infty}$ manifold,

(ii) there is a $C^{\infty}$ manifold $X$ and a $C^{\infty}$ manifold $\mathbb{M}$ with boundary $\partial \mathbb{M} \cong$ $X$ such that $M$ is the image under a continuous map $\pi: \mathbb{M} \rightarrow M$, defined by $\pi(m)=v$ for all $m \in \partial \mathbb{M}$, and $\left.\pi\right|_{\mathbb{M} \backslash \partial \mathbb{M}}: \mathbb{M} \backslash \partial \mathbb{M} \rightarrow M \backslash\{v\}$ is a diffeomorphism.

In a similar manner we can define manifolds with finitely many conical singularities $\left\{v_{1}, \ldots, v_{N}\right\}$. For simplicity we mainly consider the case $N=1$.

The space $\mathbb{M}$ is called the stretched manifold associated with $M$.

Here and in future, when we speak about topological spaces in connection with the definition of singular spaces, we assume (for convenience) that the spaces are countable unions of compact sets. In particular, our $C^{\infty}$ manifolds are assumed to be of that kind (and also oriented). Let $\mathfrak{M}_{0}$ denote the category of $C^{\infty}$ manifolds where isomorphisms (morphisms) are the diffeomorphisms (differentiable maps).

Remark 1.2 Let $\mathfrak{M}_{\text {cone }}$ denote the system of all manifolds $M$ with conical singularities. We interpret $\mathfrak{M}_{\text {cone }}$ as a category, where the isomorphisms (morphisms) $\beta: M \rightarrow \widetilde{M}$ are homeomorphisms (continuous maps) which map conical points to conical points and for which there is a diffeomorphism (differentiable map) $b: \mathbb{M} \rightarrow \widetilde{\mathbb{M}}$ of the associated stretched manifolds as $C^{\infty}$ manifolds with boundary, such that $\beta \circ \pi=\tilde{\pi} \circ b$ (where $\tilde{\pi}: \widetilde{\mathbb{M}} \rightarrow \widetilde{M}$ is of analogous meaning as the above-mentioned $\pi$ ).

Example 1.3 Let $M$ be defined to be the set $M:=\left\{\tilde{x}:=(r, x) \in \mathbb{R}^{1+n}: \tilde{x}=\right.$ 0 or $r>0, x / r \in X$ for some closed compact $C^{\infty}$ manifold $X$ embedded in $S^{n}$ \}.

Here $S^{n}$ is the unit sphere in $\mathbb{R}^{n+1} \ni \tilde{x}$. For the stretched manifold we obtain the cylinder

$$
\mathbb{M}=\left\{\tilde{x}=(r, x) \in \mathbb{R}^{1+n}: r \in \overline{\mathbb{R}}_{+}, x \in X\right\},
$$

and $\pi: \mathbb{M} \rightarrow M$ can be realised as $\pi(r, x)=(r, r x)$. 
Remark 1.4 Operators on a manifold with conical singularities are interesting for many reasons; for instance, it may be natural in a boundary value problem to assume that the boundary has conical singularities (see Kondratyev [32]). For our purposes conical singularities are crucial to forming manifolds with higher singularities, for instance, cones, where the base $X$ already has singularities.

In a second step we can pass to a wedge, i.e., the Cartesian product of that cone with a smooth manifold (see Section 1.2) and then iterate the construction (see Section 1.3).

By $H^{s}\left(\mathbb{R}^{n}\right)$ we denote the standard Sobolev space in $\mathbb{R}^{n}$ of smoothness $s \in \mathbb{R}$. On a $C^{\infty}$ manifold $X$ we have the spaces $H_{\text {comp }}^{s}(X)$ of Sobolev distributions with compact support and $H_{\text {loc }}^{s}(X) \subset \mathcal{D}^{\prime}(X)$ defined by the condition $\varphi H_{\text {loc }}^{s}(X) \subset H_{\text {comp }}^{s}(X)$ for every $\varphi \in C_{0}^{\infty}(X)$. If $X$ is closed and compact, both spaces coincide, and we simply write $H^{s}(X)$ instead of $H_{\text {comp }}^{s}(X)$.

Recall that $H^{s}\left(\mathbb{R}^{n}\right)$ can be characterised as the completion of $C_{0}^{\infty}\left(\mathbb{R}^{n}\right)$ with respect to the norm

$$
\left\{\int\langle\xi\rangle^{2 s}|F u(\xi)|^{2} d \xi\right\}^{\frac{1}{2}}
$$

where $F$ is the Fourier transform in $\mathbb{R}^{n}$. Replacing $\mathbb{R}^{n}$ by $\mathbb{R}_{+} \times \mathbb{R}^{n} \ni(r, x)$ and taking the Mellin transform on $\mathbb{R}_{+}$,

$$
(M u)(w):=\int_{0}^{\infty} r^{w-1} u(r) d r
$$

$w \in \mathbb{C}$, we obtain the weighted Sobolev space $\mathcal{H}^{s, \gamma}\left(\mathbb{R}_{+} \times \mathbb{R}^{n}\right)$, defined to be the completion of $C_{0}^{\infty}\left(\mathbb{R}_{+} \times \mathbb{R}^{n}\right)$ with respect to the norm

$$
\left\{\int_{\mathbb{R}^{n}} \frac{1}{2 \pi i} \int_{\Gamma_{\frac{n+1}{2}-\gamma}}\langle\operatorname{Re} w, \xi\rangle^{2 s}|(M F u)(w, \xi)|^{2} d w d \xi\right\}^{1 / 2} ;
$$

here $\Gamma_{\beta}:=\{w \in \mathbb{C}: \operatorname{Re} w=\beta\}$. If $X$ is a closed compact $C^{\infty}$ manifold, we then define $\mathcal{H}^{s, \gamma}\left(X^{\wedge}\right)$ for $X^{\wedge}=\mathbb{R}_{+} \times X \ni(r, x)$ by means of finite sums over contributions supported in $\mathbb{R}_{+} \times U$ for a coordinate neighbourhood $U$ (using a partition of unity on $X$ ), where the local terms are pull backs of elements of $\mathcal{H}^{s, \gamma}\left(\mathbb{R}_{+} \times \mathbb{R}^{n}\right)$ under charts $\mathbb{R}_{+} \times U$ to corresponding conical subsets of $\mathbb{R}_{+}^{1+n}=\left\{(r, x): r>0, x \in \mathbb{R}^{n}\right\}$, where the charts commute with the canonical $\mathbb{R}_{+}$-actions on $\mathbb{R}_{+} \times U$ and $\mathbb{R}_{+}^{1+n}$, respectively. An alternative definition of $\mathcal{H}^{s, \gamma}\left(X^{\wedge}\right)$ (in the notation of Remark 1.6) is given below in Theorem 3.12 (ii). 
In the sequel, by a cut-off function $\omega$ on the half-axis we understand an $\omega \in C_{0}^{\infty}\left(\overline{\mathbb{R}}_{+}\right)$that is equal to 1 in a neighbourhood of 0 .

In an infinite (stretched) cone with closed compact base $X$ there is also another species of weighted spaces, namely,

$$
\mathcal{K}^{s, \gamma}\left(X^{\wedge}\right):=\left\{\omega u+(1-\omega) v: u \in \mathcal{H}^{s, \gamma}\left(X^{\wedge}\right), v \in H_{\text {cone }}^{s}\left(X^{\wedge}\right)\right\} .
$$

For purposes below we also define the spaces

$$
\mathcal{K}^{s, \gamma ; g}\left(X^{\wedge}\right):=\langle r\rangle^{-g} \mathcal{K}^{s, \gamma}\left(X^{\wedge}\right)
$$

or any $s, \gamma, g \in \mathbb{R}$.

Here, for $X=S^{n}$ the space $H_{\text {cone }}^{s}\left(X^{\wedge}\right)$ is defined to be the subspace of all $\left.u \in H_{\text {loc }}^{s}(\mathbb{R} \times X)\right|_{\mathbb{R}_{+} \times X}$ such that $(1-\omega) u$ in the Euclidean coordinates of $\mathbb{R}_{\tilde{x}}^{1+n}$ (where $(r, x) \in \mathbb{R}_{+} \times X$ are polar coordinates in $\mathbb{R}_{\tilde{x}}^{1+n} \backslash\{0\}$ ) belongs to $H^{s}\left(\mathbb{R}^{1+n}\right)$. If $X$ is an arbitrary closed compact $C^{\infty}$ manifold, we define $H_{\text {cone }}^{s}\left(X^{\wedge}\right)$ to be the set of all $\left.u \in H_{\text {loc }}^{s}(\mathbb{R} \times X)\right|_{\mathbb{R}_{+} \times X}$ such that $(1-\omega) \varphi u$ is the pull back of an element in $H^{s}\left(\mathbb{R}_{\tilde{x}}^{1+n}\right)$, for any $\varphi \in C_{0}^{\infty}(U)$ for an arbitrary coordinate neighbourhood $U$ on $X$, where the pull back refers to a diffeomorphism $\mathbb{R}_{+} \times U \rightarrow \Gamma$ for a conical set $\Gamma \subset \mathbb{R}_{\tilde{x}}^{1+n}$ which commutes with the canonical $\mathbb{R}_{+}$-actions on $\mathbb{R}_{+} \times U$ and $\mathbb{R}_{+}^{1+n}$, respectively (the one in $\mathbb{R}_{+}^{1+n}$ is $\tilde{x} \rightarrow \lambda \tilde{x}$, the multiplication by $\lambda \in \mathbb{R}_{+}$). Concerning more observations on the spaces $H_{\text {cone }}^{s}$, see Remark 2.20 and Theorem 3.13 below.

Remark 1.5 The spaces $\mathcal{K}^{s, \gamma ; g}\left(X^{\wedge}\right)$ are Hilbert spaces in a natural way. They can be endowed with a family of isomorphisms $\kappa_{\lambda}^{g}: \mathcal{K}^{s, \gamma ; g}\left(X^{\wedge}\right) \rightarrow$ $\mathcal{K}^{s, \gamma ; g}\left(X^{\wedge}\right), u(r, x) \rightarrow \lambda^{\frac{n+1}{2}+g} u(\lambda r, x), \lambda \in \mathbb{R}_{+}$.

In general, if $H$ is a Hilbert space and $\kappa=\left\{\kappa_{\lambda}\right\}_{\lambda \in \mathbb{R}_{+}}$a group of isomorphisms

$$
\kappa_{\lambda}: H \rightarrow H, \lambda \in \mathbb{R}_{+},
$$

$\kappa_{\lambda} \kappa_{\nu}=\kappa_{\lambda \nu}$ for arbitrary $\lambda, \nu \in \mathbb{R}$, and if $\lambda \rightarrow \kappa_{\lambda} h$ defines a continuous map $\mathbb{R}_{+} \rightarrow H$ for every $h \in H$ (i.e., $\kappa$ is strongly continuous), then we say that $H$ is endowed with a group action.

Remark 1.6 Later on, in order to point out the cone $X^{\Delta}$ itself instead of the open stretched cone $X^{\wedge}$, we often write $H_{\text {cone }}^{s}\left(X^{\Delta}\right):=H_{\text {cone }}^{s}\left(X^{\wedge}\right)$, $\mathcal{H}^{s, \gamma}\left(X^{\Delta}\right):=\mathcal{H}^{s, \gamma}\left(X^{\wedge}\right)$, and

$$
\mathcal{K}^{s, \gamma ; g}\left(X^{\Delta}\right):=\mathcal{K}^{s, \gamma ; g}\left(X^{\wedge}\right), K^{s, \gamma}\left(X^{\Delta}\right):=\mathcal{K}^{s, \gamma ; s-\gamma}\left(X^{\wedge}\right) .
$$

Nevertheless all those spaces are distribution spaces over $X^{\wedge}=X^{\Delta} \backslash\{$ tip of the cone $\}$. 
Finally, if $M$ is a compact manifold with conical singularity $\{v\}$, we define the space $H^{s, \gamma}(M)$ to be the subspace of all $u \in H_{\text {loc }}^{s}(M \backslash\{v\})$ such that $\omega u \in \mathcal{H}^{s, \gamma}\left(X^{\wedge}\right)$ for any continuous function $\omega$ on $M$ supported in a small neighbourhood of $v$ such that $\left.\omega\right|_{M \backslash\{v\}}$ is $C^{\infty}$ and $\omega \equiv 1$ in another neighbourhood of $v$. The meaning of $X$ is as in Definition 1.1, and the condition on $\omega u$ refers to the splitting of variables $(r, x) \in X^{\wedge}$ in $M \backslash\{v\}$ close to the point $v$.

An $A \in \operatorname{Diff}^{\mu}(M \backslash\{v\})$ is said to be of Fuchs type, written $A \in \operatorname{Diff}_{\operatorname{deg}}^{\mu}(M)$, if $A$ locally near $v$ in the splitting of variables $(r, x) \in X^{\wedge}$ close to $v$ has the form

$$
A=r^{-\mu} \sum_{j=0}^{\mu} a_{j}(r)\left(-r \frac{\partial}{\partial r}\right)^{j}
$$

with coefficients $a_{j} \in C^{\infty}\left(\overline{\mathbb{R}}_{+}, \operatorname{Diff}^{\mu-j}(X)\right)$.

Observe that when $M$ is compact, every $A \in \operatorname{Diff}_{\mathrm{deg}}^{\mu}(M)$ induces continuous operators

$$
A: H^{s, \gamma}(M) \rightarrow H^{s-\mu, \gamma-\mu}(M)
$$

for all $s, \gamma \in \mathbb{R}$.

For any $A \in \operatorname{Diff}_{\mathrm{deg}}^{\mu}(M)$ we define

$$
\sigma(A):=\left(\sigma_{\psi}(A), \sigma_{\mathrm{c}}(A)\right) .
$$

The first component is the homogeneous principal symbol

$$
\sigma_{\psi}(A):=\sigma_{\psi}\left(\left.A\right|_{M \backslash\{v\}}\right)
$$

Observe that locally close to $v$ in the splitting of variables $(r, x) \in X^{\wedge}$ with the covariables $(\varrho, \xi)$ the symbol $\sigma_{\psi}(A)$ has the form

$$
\sigma_{\psi}(A)(r, x, \varrho, \xi)=r^{-\mu} \tilde{\sigma}_{\psi}(A)(r, x, r \varrho, \xi)
$$

for a $\tilde{\sigma}_{\psi}(A)(r, x, \varrho, \xi)$ which is smooth up to $r=0$. The second component is the principal conormal symbol

$$
\sigma_{\mathrm{c}}(A)(w):=\sum_{j=0}^{\mu} a_{j}(0) w^{j},
$$

interpreted as a family of differential operators on

$$
\sigma_{\mathrm{c}}(A)(w): H^{s}(X) \rightarrow H^{s-\mu}(X),
$$

parametrised by $w \in \Gamma_{\frac{n+1}{2}-\gamma}$ for any fixed $\gamma$. 
Definition 1.7 An operator $A \in \operatorname{Diff}_{\mathrm{deg}}^{\mu}(M)$ is said to be elliptic (with respect to a given weight $\gamma$ ) if

(i) $A$ is $\sigma_{\psi}$-elliptic, i.e., $\sigma_{\psi}(A) \neq 0$ on $T^{*}(M \backslash\{v\}) \backslash 0$ and in addition $\tilde{\sigma}_{\psi}(A) \neq 0$ for $(\varrho, \xi) \neq 0$, up to $r=0$;

(ii) the operators (10), $w \in \Gamma_{\frac{n+1}{2}-\gamma}$, define a family of isomorphisms for some $s=s_{0} \in \mathbb{R}$.

Remark 1.8 Let $A \in \operatorname{Diff}_{\operatorname{deg}}^{\mu}(M)$ be $\sigma_{\psi}$-elliptic. Then there is a discrete set $D \subset \mathbb{C}$ (i.e., $D$ is countable and $K \cap D$ finite for every compact set $K \subset \mathbb{C}$ ) such that the operators (10) are isomorphisms for all $w \in \mathbb{C} \backslash D$ for every $s \in \mathbb{R}$. This property determines all $\gamma \in \mathbb{R}$ where Definition 1.7 is satisfied, namely, when $D \cap \Gamma_{\frac{n+1}{2}-\gamma}=\emptyset$.

Theorem 1.9 For an operator $A \in \operatorname{Diff}_{\mathrm{deg}}^{\mu}(M)$ the following conditions are equivalent:

(i) A is elliptic (with respect to a weight $\gamma$ );

(ii) the operator (7) is Fredholm for some $s_{0}=s \in \mathbb{R}$.

If A satisfies the condition (i), then (7) is Fredholm for all $s \in \mathbb{R}$; moreover $V=\operatorname{ker} A \subset H^{\infty, \gamma}(M)$ is independent of $s$, and there is a subspace $W \subset$ $H^{\infty, \gamma-\mu}(M)$ of finite dimension such that $W \cap \operatorname{im} A=\{0\}$, and

$$
W+\operatorname{im} A=H^{s-\mu, \gamma-\mu}(M)
$$

for every $s \in \mathbb{R}$.

The problem of expressing parametrices of elliptic operators in $\operatorname{Diff}^{\mu}(M)$ gives rise to what we call the cone algebra on $M$. By that we understand a specific pseudo-differential calculus, consisting of subspaces $\mathfrak{A}^{\mu}(M,(\gamma, \gamma-$ $\mu)) \subset L_{\mathrm{cl}}^{\mu}(M \backslash\{v\})$ (see Sections 2.1, 2.2, 3.1 below) of operators $A$ with a principal symbolic structure of the form (6). Every $A \in \mathfrak{A}^{\mu}(M,(\gamma, \gamma-\mu))$ induces continuous operators (5). Below we shall develop some ingredients of the cone algebra. More details may be found in various papers and monographs of the author, see [69], [72], [74], and other references mentioned in the introduction.

An essential element is the elliptic regularity of solutions.

Theorem 1.10 Let $A \in \mathfrak{A}^{\mu}(M,(\gamma, \gamma-\mu)), \mu \in \mathbb{R}$, be elliptic (in the pseudodifferential set-up, similarly defined as in Definition 1.7). Then there is a parametrix $B \in \mathfrak{A}^{-\mu}(M,(\gamma-\mu, \gamma))$ such that $1-B A$ and $1-A B$ belong to $\mathfrak{A}^{-\infty}(M,(\gamma, \gamma))$ and $\mathfrak{A}^{-\infty}(M,(\gamma-\mu, \gamma-\mu))$, respectively. 
Here the spaces $\mathfrak{A}^{-\infty}$ consist of smoothing operators which are compact in the respective weighted spaces (when $M$ is compact) and with specific mapping properties referring to asymptotics close the conical points, see the considerations below. A consequence is that

$$
A u=f \in H^{s-\mu, \gamma-\mu}(M), u \in H^{-\infty, \gamma}(M)
$$

implies $u \in H^{s, \gamma}(M)$, for every $s \in \mathbb{R}$.

The result follows from $B A u=B f \in H^{s, \gamma}(M)$ and $B A u=u-G u$, $G u \in H^{-\infty, \gamma}(M)$.

The precise definition of $\mathfrak{A}^{-\infty}(M,(\gamma, \gamma-\mu)) \ni G$ consists of requiring the continuity property

$$
G: H^{s, \gamma}(M) \rightarrow H_{P}^{\infty, \gamma-\mu}(M)
$$

for all $s \in \mathbb{R}$, with a so-called asymptotic type $P$

$$
P=\left\{\left(p_{j}, m_{j}, L_{j}\right)\right\}_{j \in \mathbb{N}} .
$$

A similar condition is asked for $G^{*}$, the formal adjoint with respect to the $H^{0,0}(M)$-scalar product. The definition of $H_{P}^{s, \gamma}(M)$ is as follows. By discrete asymptotics of a function $u \in K^{s, \gamma}\left(X^{\Delta}\right)$ of type $P$ for $r \rightarrow 0$ we understand the property that for every $\beta>0$ there exists an $N=N(\beta) \in \mathbb{N}$ such that for any cut-off function $\omega$

$$
v_{N}(r, x):=u(r, x)-\omega(r) \sum_{j=0}^{N} \sum_{k=0}^{m_{j}} c_{j k}(x) r^{-p_{j}} \log ^{k} r \in K^{s, \beta}\left(X^{\Delta}\right)
$$

with $p_{j} \in \mathbb{C}, \operatorname{Re} p_{j}<\frac{n+1}{2}-\gamma, \operatorname{Re} p_{j} \rightarrow-\infty$ as $N \rightarrow \infty$ (when there are infinitely many $\left.p_{j}\right), k \in \mathbb{N}$, and coefficients $c_{j k}$ belonging to finite-dimensional subspaces $L_{j} \subset C^{\infty}(X)$. We usually assume the 'shadow condition' which means $(p, m, L) \in P \Rightarrow(p-j, m, L) \in P$ for all $j \in \mathbb{N}$.

The space $K_{P}^{s, \gamma}\left(X^{\Delta}\right)$ is Fréchet with a semi-norm system consisting of $u \rightarrow\left\|v_{N}\right\|_{\mathcal{K}^{s, \beta}\left(X^{\Delta}\right)}$ for every $\beta>0$ and $u \rightarrow\left\|c_{N}\right\|_{\mathbb{C}^{L(N)}}, N=N(\beta)$, with $c_{N}=c_{N}(u)$ being the (unique) sequence of coefficients $\left(c_{j k}\right)_{0 \leq k \leq m_{j}, 0 \leq j \leq N} \in$ $\oplus_{j=1}^{N} L_{j}$, where the latter space is identified with $\mathbb{C}^{L(N)}$ for $L(N)=\sum_{j=0}^{N}\left(m_{j}+\right.$ 1) $\operatorname{dim} L_{j}$. Flatness of order $\Theta$ relative to the weight $\gamma$ is measured by functions in the Fréchet space

$$
K_{\Theta}^{s, \gamma}\left(X^{\Delta}\right):=\lim _{k \in \mathbb{N}} K^{s, \gamma-\vartheta-(k+1)^{-1}}\left(X^{\Delta}\right),
$$

here $\Theta$ represents the half-open 'weight interval' $\Theta=(-\vartheta, 0],-\infty \leq \vartheta<0$, The (non-direct) sum

$$
K_{P_{\Theta}}^{s, \gamma}\left(X^{\Delta}\right):=K_{P}^{s, \gamma}\left(X^{\Delta}\right)+K_{\Theta}^{s, \gamma}\left(X^{\Delta}\right)
$$


gives us a notion of finite asymptotic expansions of the respective $u(r, x)$, (when $\vartheta>-\infty$ ) with $p_{j}$ belonging to the weight strip $\left\{\frac{n+1}{2}-\gamma+\vartheta<\right.$ $\left.\operatorname{Re} w<\frac{n+1}{2}-\gamma\right\}, n=\operatorname{dim} X$. Observe that $\omega(r) r^{-p} \log ^{k} r c(x) \in \mathcal{K}_{\Theta}^{s, \gamma}\left(X^{\Delta}\right)$ for every $p \in \mathbb{C}$ with $\operatorname{Re} p \leq \frac{n+1}{2}-\gamma+\vartheta, k \in \mathbb{N}, c \in C^{\infty}(X)$.

Now if $M$ is a manifold with conical singularity $\{v\}$, we fix (as an additional datum) a splitting of variables $(r, x) \in \mathbb{R}_{+} \times X$ close to $\{v\}$. Then we can identify $\omega u \in K_{P}^{s, \gamma}\left(X^{\Delta}\right)$ for a cut-off function $\omega$ with a corresponding element in $H^{s, \gamma}(M)$, and we then denote by $H_{P}^{s, \gamma}(M)$ the subspace of all $u \in H^{s, \gamma}(M)$ that localise near $v$ to such a function with asymptotics of type $P$.

Remark 1.11 Given an elliptic (with respect to a weight $\gamma \in \mathbb{R}$ ) operator $A \in \operatorname{Diff}_{\mathrm{deg}}^{\mu}(M)$ then

$$
A u=f \in H_{Q}^{s-\mu, \gamma-\mu}(M), u \in H^{-\infty, \gamma}(M)
$$

implies $u \in H_{P}^{s, \gamma}(M)$ for every discrete asymptotic type $Q$ with a resulting discrete asymptotic type $P$. The components of $P$ are determined by $Q$ and by $\sigma_{\mathrm{c}}(A)^{-1}(w)$ which is a meromorphic Fredholm family, with poles of finite multiplicity and finite rank Laurent coefficients at the principal part of the Laurent expansion. A similar result is true of elliptic operators $A \in$ $\mathfrak{A}^{\mu}(M,(\gamma, \gamma-\mu))$ in general.

Remark 1.12 The latter statement refers to the cone algebra with discrete asymptotics. In the edge and the higher corner calculus below we prefer to generalise the notion of asymptotics as continuous asymptotics.

The essential aspect is to rephrase the singular functions of asymptotics in terms of analytic functionals in the complex plane of the Mellin covariable $w$. Examples of such functionals are linear combinations

$$
\zeta_{N}:\left.h \rightarrow \sum_{j=0}^{N} \sum_{k=0}^{m_{j}} l_{j k} \frac{d^{k}}{d w^{k}} h(w)\right|_{w=p_{j}}
$$

with (in the scalar case) coefficients $l_{j k} \in \mathbb{C}, h \in \mathcal{A}(\mathbb{C})$. (Here $\mathcal{A}(U)$ for any open $U \subset \mathbb{C}$ denotes the space of all holomorphic functions in $U$, in the (nuclear) Fréchet topology of uniform convergence on compact subsets; moreover, if $F$ is a Fréchet space, then $\mathcal{A}(U, F)$ denotes the space of all holomorphic $F$-valued functions in $U$ in the topology of the projective tensor product $\left.\mathcal{A}(U) \hat{\otimes}_{\pi} F\right)$.

More generally, if $K \subset \mathbb{C}$ is a compact set and $C$ a (say, smooth) compact curve clockwise surrounding $K$ (such that the winding number with respect 
to every point of $K$ is equal to 1$)$, then every $f \in \mathcal{A}(\mathbb{C} \backslash K)$ determines an analytic functional

$$
\zeta: h \rightarrow \frac{1}{2 \pi i} \int_{C} h(w) f(w) d w .
$$

For $f$ in (13) we can take a meromorphic function with poles at the $p_{j} \in \mathbb{C}$ of multiplicity $m_{j}+1$ and suitable Laurent coefficients. Taking $h(w)=r^{-w}$ we obtain

$$
\left\langle\zeta_{N}, r^{-w}\right\rangle=\sum_{j=0}^{N} \sum_{k=0}^{m_{j}} c_{j k} r^{-p_{j}} \log ^{k} r
$$

for $c_{j k}=k !(-1)^{k} l_{j k}$. This shows that discrete asymptotics of a function $u(r)$ in $K_{P}^{s, \gamma}\left(\overline{\mathbb{R}}_{+}\right)$can be expressed by the condition that

$$
u-\omega(r)\left\langle\zeta_{N}, r^{-w}\right\rangle
$$

belongs to $K^{s, \beta}\left(\overline{\mathbb{R}}_{+}\right)$, for every $\beta>0$ with a suitable $\zeta_{N}$ of the kind (13), $N=N(\beta) \in \mathbb{N}$.

The generalisation to an arbitrary base $X$ of the cone is evident. In (13) it suffices to assume $l_{j k} \in L_{j}, 0 \leq k \leq m_{j}$, i.e., to talk about $C^{\infty}(X)$ valued analytic functionals. In (14) in this generalisation we would take $f \in \mathcal{A}\left(\mathbb{C} \backslash K, C^{\infty}(X)\right)$. Let $\mathcal{A}^{\prime}\left(K, C^{\infty}(X)\right)$ denote the (nuclear Fréchet) space of $C^{\infty}(X)$-valued analytic functionals carried by $K$.

Let $P \subset \mathbb{C}$ be a closed set such that $P \subset\left\{\operatorname{Re} w<\frac{n+1}{2}-\gamma\right\}, P \cap\left\{c_{0}<\right.$ $\left.\operatorname{Re} w<c_{1}\right\}$ compact for every $c_{0}<c_{1}$, and $w_{0}, w_{1} \in P$, Re $w_{0}=\operatorname{Re} w_{1} \Rightarrow$ $(1-\lambda) w_{0}+\lambda w_{1} \in P$ for every $0 \leq \lambda \leq 1$. We then call $P$ a continuous asymptotic type associated with the weight data $(\gamma, \Theta), \Theta=(-\infty, 0]$. If $w \in P \Rightarrow w-j \in P$ for every $j \in \mathbb{N}$, we say that $P$ satisfies the shadow condition (this will be tacitly assumed in the sequel).

Definition 1.13 We define $K_{P}^{s, \gamma}\left(X^{\Delta}\right)$ to be the subspace of all $u \in K^{s, \gamma}\left(X^{\Delta}\right)$ such that for every $\beta>0$ there is an $N \in \mathbb{N}$ and $a \zeta_{N} \in \mathcal{A}^{\prime}\left(P \cap\left\{\operatorname{Re} w \geq \frac{n+1}{2}-\right.\right.$ $\left.2 \beta\}, C^{\infty}(X)\right)$, such that the remainder (15) belongs to $K^{s, \beta}\left(X^{\Delta}\right)$. Similarly as (12) we can form spaces with continuous asymptotics in a finite weight interval $\Theta=(-\vartheta, 0]$ relative to $\gamma$.

The spaces $K_{P}^{s, \gamma}\left(X^{\Delta}\right)$ are Fréchet in a natural way; then also (12) is Fréchet in the topology of the non-direct sum.

Similarly as in the discrete case we can form (Fréchet) spaces $H_{P}^{s, \gamma}(M)$ of weighted distributions with continuous asymptotics of type $P$ on a manifold $M$ with conical singularity $v$ (and also $H_{P_{\Theta}, \gamma}^{s,}(M)$ for arbitrary $\Theta$ ). This gives rise to a more general notion of Green operators (11) in the cone algebra, namely, Green operators with continuous asymptotics. 


\subsection{Boundaries and edges}

Definition 1.14 A manifold $M$ with edge is a topological space with a subset $Y \subset M$, the edge, such that

(i) $M \backslash Y$ and $Y$ are $C^{\infty}$ manifolds;

(ii) there is a $C^{\infty}$ manifold $X$ and a $C^{\infty}$ manifold $\mathbb{M}$ with boundary $\partial \mathbb{M}$ such that $\partial \mathbb{M}$ has the structure of an $X$-bundle over $Y$, and $M$ is the image under a continuous map $\pi: \mathbb{M} \rightarrow M$, where $\left.\pi\right|_{\partial \mathbb{M}}$ is the bundle projection $\partial \mathbb{M} \rightarrow Y$, and $\left.\pi\right|_{\mathbb{M} \backslash \partial \mathbb{M}}: \mathbb{M} \backslash \partial \mathbb{M} \rightarrow M \backslash Y$ is a diffeomorphism.

The space $\mathbb{M}$ is called the stretched manifold associated with $M$.

For simplicity we assume that $Y$ has only one connected component, $\operatorname{dim} Y=q$. The case $q=0$ corresponds to a conical singularity. In a similar manner we can consider the case of finitely many connected components of different dimension.

Remark 1.15 Let $\mathfrak{M}_{1}$ denote the system of all manifolds $M$ with edge singularity. We interpret $\mathfrak{M}_{1}$ as a category, where the isomorphisms (morphisms) $\beta: M \rightarrow \widetilde{M}$ are homeomorphisms (continuous maps) which restrict to diffeomorphisms (differentiable maps) $Y \rightarrow \widetilde{Y}$ between the respective edges and for which there is a diffeomorphism (differentiable map) $b: \mathbb{M} \rightarrow \widetilde{\mathbb{M}}$ of the associated stretched manifolds as $C^{\infty}$ manifolds with boundary, such that $\beta \circ \pi=\tilde{\pi} \circ b$ (where $\tilde{\pi}: \widetilde{\mathbb{M}} \rightarrow \widetilde{M}$ is of analogous meaning as the above-mentioned $\pi$ ), and $b$ restricts to a bundle isomorphism (morphism) $\partial \mathbb{M} \rightarrow \partial \widetilde{\mathbb{M}}$ of the $X$ bundle over $Y$ to the respective $\widetilde{X}$ bundle over $\widetilde{Y}$.

Note that $\mathfrak{M}_{\text {cone }}$ is a subcategory of $\mathfrak{M}_{1}$.

Example 1.16 (i) Let $X$ be a $C^{\infty}$ manifold and $X^{\Delta}=\left(\overline{\mathbb{R}}_{+} \times X\right) /(\{0\} \times$ $X)$ the infinite cone with base $X$, interpreted as an element of $\mathfrak{M}_{\text {cone }}$ with $\overline{\mathbb{R}}_{+} \times X$ as the associated stretched manifold. Then, if $\Omega \subseteq \mathbb{R}^{q}$ is an open set, the wedge

$$
M:=X^{\Delta} \times \Omega
$$

is a manifold with edge $\Omega$. The associated stretched manifold is equal to

$$
\mathbb{M}=\overline{\mathbb{R}}_{+} \times X \times \Omega .
$$

The boundary $\partial \mathbb{M}$ in this case is the trivial $X$-bundle $X \times \Omega$.

(ii) Let $M$ be a $C^{\infty}$ manifold with boundary. Then the boundary can be interpreted as an edge $Y$, and the $C^{\infty}$ manifold $X$ in Definition 1.14 is of dimension 0 . In this case $M$ is equal to its stretched manifold $\mathbb{M}$. 
Remark 1.17 From Definition 1.14 it follows that when $M$ is a manifold with edge $Y$, every $y \in Y$ has a neighbourhood $V \in \mathfrak{M}_{1}$ such that there is an isomorphism

$$
\chi: V \rightarrow X^{\Delta} \times \Omega
$$

in $\mathfrak{M}_{1}$ for an open set $\Omega \subseteq \mathbb{R}^{q}, q=\operatorname{dim} Y$; in particular, $\chi$ is associated with a diffeomorphism

$$
\mathbb{V} \rightarrow \overline{\mathbb{R}}_{+} \times X \times \Omega
$$

between the associated stretched manifolds. In addition $\chi$ restricts to diffeomorphisms

$$
V \backslash Y \rightarrow X^{\wedge} \times \Omega \text { and } V \cap Y \rightarrow \Omega .
$$

The manifold $X$ will also be called the base of the (local) model cone $X^{\Delta}$ of $M$. If $M$ is a manifold with $C^{\infty}$ boundary, then we may write $\mathbb{V}=V$, and (16) corresponds to the transformation of a neighbourhood $V$ of a point $y \in \partial M$ to the half-space $\overline{\mathbb{R}}_{+} \times \Omega$.

According to these observations to any point of the edge we fix such a neighbourhood $V$ and tacitly identify $V \backslash$ \{edge $\}$ with the open stretched wedge $X^{\wedge} \times \Omega$ in the splitting of variables $(r, x, y)$.

Let $M$ be a manifold with edge $Y$, and let $X$ be the base of the model cone. An $A \in \operatorname{Diff}^{\mu}(M \backslash Y)$ is said to be edge-degenerate, written $A \in \operatorname{Diff}_{\operatorname{deg}}^{\mu}(M)$, if locally near any point of $Y$ in the splitting of variables $(r, x, y) \in X^{\wedge} \times \Omega$ the operator $A$ has the form

$$
A=r^{-\mu} \sum_{j+|\alpha| \leq \mu} a_{j \alpha}(r, y)\left(-r \frac{\partial}{\partial r}\right)^{j}\left(r D_{y}\right)^{\alpha}
$$

with coefficients $a_{j \alpha} \in C^{\infty}\left(\overline{\mathbb{R}}_{+} \times \Omega\right.$, Diff $\left.{ }^{\mu-(j+|\alpha|)}(X)\right)$. For any $A \in \operatorname{Diff}_{\mathrm{deg}}^{\mu}(M)$ we define

$$
\sigma(A):=\left(\sigma_{\psi}(A), \sigma_{\wedge}(A)\right)
$$

The first component is the homogeneous principal symbol $\sigma_{\psi}(A):=\sigma_{\psi}\left(\left.A\right|_{M \backslash Y}\right)$.

Observe that locally close to the edge in the splitting of variables $(r, x, y) \in$ $X^{\wedge} \times \Omega$ with the covariables $(\varrho, \xi, \eta)$ the symbol $\sigma_{\psi}(A)$ has the form

$$
\sigma_{\psi}(A)(r, x, y, \varrho, \xi, \eta)=r^{-\mu} \tilde{\sigma}_{\psi}(A)(r, x, y, r \varrho, \xi, r \eta)
$$

for a $\tilde{\sigma}_{\psi}(A)(r, x, y, \varrho, \xi, \eta)$ which is smooth up to $r=0$.

The second component of (19) is the homogeneous principal edge symbol

$$
\sigma_{\wedge}(A)(y, \eta):=r^{-\mu} \sum_{j+|\alpha| \leq \mu} a_{j \alpha}(0, y)\left(-r \frac{\partial}{\partial r}\right)^{j}(r \eta)^{\alpha},
$$

considered for $(y, \eta) \in \Omega \times\left(\mathbb{R}^{q} \backslash\{0\}\right)$. 
Proposition 1.18 Let $X$ in Definition 1.14 be a closed compact $C^{\infty}$ manifold; then the principal edge symbol of $A \in \operatorname{Diff}_{\mathrm{deg}}^{\mu}(M)$ defines a family of continuous operators

$$
\sigma_{\wedge}(A)(y, \eta): \mathcal{K}^{s, \gamma ; g}\left(X^{\Delta}\right) \rightarrow \mathcal{K}^{s-\mu, \gamma-\mu ; g}\left(X^{\Delta}\right)
$$

for every $s, g, \gamma \in \mathbb{R}$, smoothly depending on $y, \eta$.

Remark 1.19 We have

$$
\sigma_{\wedge}(A)(y, \lambda \eta)=\lambda^{\mu} \kappa_{\lambda} \sigma_{\wedge}(A)(y, \eta) \kappa_{\lambda}^{-1} \text { for all } \lambda \in \mathbb{R}_{+}
$$

(see Remark 1.20). This property is also referred to as twisted homogeneity.

Remark 1.20 Observe that the operators $\sigma_{\wedge}(A)(y, \eta)$ for every fixed $y$ and $\eta \neq 0$ represent an element of $\operatorname{Diff}_{\mathrm{deg}}^{\mu}\left(X^{\Delta}\right)$, (see the notation in Section 1.1). Therefore, we have the 'subordinate' symbols from (8), especially, the conormal symbol

$$
\sigma_{\mathrm{c}} \sigma_{\wedge}(A)(y, w)=\sum_{j=0}^{\mu} a_{j 0}(0, y) w^{j}: H^{s}(X) \rightarrow H^{s-\mu}(X) .
$$

Definition 1.21 An operator $A=\operatorname{Diff}_{\mathrm{deg}}^{\mu}(M)$ is said to be $\sigma_{\psi}$-elliptic, if $\sigma_{\psi}(A) \neq 0$ on $T^{*}(M \backslash Y) \backslash 0$ and if in addition $\tilde{\sigma}_{\psi}(A) \neq 0$ for $(\varrho, \xi, \eta) \neq 0$, up to $r=0$ for any wedge neighbourhood $V$ of a point the edge.

Remark 1.22 If $A \in \operatorname{Diff}_{\operatorname{deg}}^{\mu}(M)$ is $\sigma_{\psi}$-elliptic, then for every fixed $y$ and $\eta \neq 0$ the operator $\sigma_{\wedge}(A)(y, \eta) \in \operatorname{Diff}_{\mathrm{deg}}^{\mu}\left(X^{\Delta}\right)$ is $\sigma_{\psi}$-elliptic in the sense of Definition 1.7 (i). Moreover, according to Remark 1.8 for every $y \in \Omega$ there is a discrete set $D(y) \subset \mathbb{C}$ such that the operators (24) are isomorphisms for all $w \in \Gamma_{\frac{n+1}{2}-\gamma}$ when $D(y) \cap \Gamma_{\frac{n+1}{2}-\gamma}=\emptyset$, for every $s \in \mathbb{R}$.

Theorem 1.23 Let $M$ be a manifold with edge, $X$ compact, and let $A \in$ $\operatorname{Diff}_{\mathrm{deg}}^{\mu}(M)$ be $\sigma_{\psi}$-elliptic. Then the operators (22) are Fredholm for all $y \in \Omega$, $\eta \neq 0$, and all $\gamma=\gamma(y) \in \mathbb{R}$ where $D(y) \cap \Gamma_{\frac{n+1}{2}-\gamma}=\emptyset$; this holds for all $s, g \in \mathbb{R}$. For $\gamma=\gamma(y)$ we have $V=\operatorname{ker} \sigma_{\wedge}(A)(y, \eta) \subset \mathcal{K}^{\infty, \gamma ; \infty}\left(X^{\wedge}\right)$ which is independent of $s$, and there is a subspace $W \subset \mathcal{K}^{\infty, \gamma-\mu ; \infty}\left(X^{\Delta}\right)$ of finite dimension such that $W \cap \operatorname{im} \sigma_{\wedge}(A)(y, \eta)=\{0\}$ and $W+\operatorname{im} \sigma_{\wedge}(A)(y, \eta)=$ $\mathcal{K}^{s-\mu, \gamma-\mu ; g}\left(X^{\Delta}\right)$ for every $s, g \in \mathbb{R}, \eta \neq 0$.

We shall return to the nature of edge symbols as operators on an infinite cone, in the context of conical exits to infinity, see Theorem 2.21 below.

Let us now make a few remarks on the case when $M$ is a $C^{\infty}$ manifold with boundary. As noted in the introduction (and as everybody knows) the 
ellipticity of operators is usually combined with the ellipticity of boundary conditions.

The local model of a manifold with boundary is the half-space $\overline{\mathbb{R}}_{+} \times \Omega \ni$ $(r, y), \Omega \subseteq \mathbb{R}^{q}$ open, i.e., a wedge $X^{\Delta} \times \Omega$ with $\operatorname{dim} X=0$. In 'standard' boundary value problems the natural differential operators are usually not assumed to be of the form (18) but

$$
A=\sum_{j+|\alpha| \leq \mu} b_{j \alpha}(r, y) D_{r}^{j} D_{y}^{\alpha}
$$

with $b_{j \alpha} \in C^{\infty}\left(\overline{\mathbb{R}}_{+} \times \Omega\right)$. Clearly we can easily transform (25) into the form (18), but the class of edge-degenerate operators is much larger than the class of operators (21). For simplicity, in order to avoid 'comp' or 'loc' Sobolev spaces, we assume $\Omega=\mathbb{R}^{q}$ and $b_{j \alpha}(r, y)$ independent of $(r, y)$ for $|r, y|>C$ for some $C>0$. Then (25) is continuous as an operator

$$
A: H^{s}\left(\mathbb{R}_{+}^{1+q}\right) \rightarrow H^{s-\mu}\left(\mathbb{R}_{+}^{1+q}\right)
$$

$H^{s}\left(\mathbb{R}_{+}^{1+q}\right)=\left.H^{s}\left(\mathbb{R}^{1+q}\right)\right|_{\mathbb{R}_{+}^{1+q}}, \mathbb{R}_{+}^{1+q}=\left\{(r, y) \in \mathbb{R}^{1+q}: r>0, y \in \mathbb{R}^{q}\right\}$. As before, the operator $A$ has its principal symbol $\sigma_{\psi}(A)(r, y, \varrho, \eta)=\sum_{j+|\alpha|=\mu} b_{j \alpha}(r, y) \varrho^{j} \eta^{\alpha}$, and an analogue of the principal edge symbol (21) is the principal boundary symbol

$$
\sigma_{\partial}(A)(y, \eta):=\sigma_{\psi}(A)\left(0, y, D_{r}, \eta\right): H^{s}\left(\mathbb{R}_{+}\right) \rightarrow H^{s-\mu}\left(\mathbb{R}_{+}\right)
$$

acting between standard Sobolev spaces on the half-axis, $\eta \neq 0$.

The spaces $H^{s}\left(\mathbb{R}_{+}\right)$are Hilbert spaces with group action $\left(\kappa_{\lambda} u\right)(r):=$ $\lambda^{\frac{1}{2}} u(\lambda r), \lambda \in \mathbb{R}_{+}$, and the homogeneity of the boundary symbol is similar to (23), namely,

$$
\sigma_{\partial}(A)(y, \lambda \eta)=\lambda^{\mu} \kappa_{\lambda} \sigma_{\partial}(A)(y, \eta) \kappa_{\lambda}^{-1}
$$

for all $\lambda \in \mathbb{R}_{+}$.

Remark 1.24 Let (25) be elliptic, i.e., $\sigma_{\psi}(A)(r, y, \varrho, \eta) \neq 0$ for $(\varrho, \eta) \neq 0$; then (27) is a surjective family of Fredholm operators for every $\eta \neq 0$ and $s>\mu-\frac{1}{2}$.

Example 1.25 Consider the Laplacian $\Delta=\frac{\partial^{2}}{\partial r^{2}}+\sum_{j=1}^{q} \frac{\partial^{2}}{\partial y_{j}^{2}}$. Then

$$
\sigma_{\psi}(\Delta)(\varrho, \eta)=-|\varrho|^{2}-|\eta|^{2} \text { and } \sigma_{\partial}(\Delta)(\eta)=\frac{\partial^{2}}{\partial r^{2}}-|\eta|^{2}
$$

and we have $\operatorname{ker} \sigma_{\partial}(\Delta)(\eta)=\left\{c e^{-|\eta| r}: c \in \mathbb{C}\right\}$. 
A boundary value problem for an elliptic operator $A$ consists of the task to solve the equations

$$
A u=f \text { in the domain, } T u=g \text { on the boundary, }
$$

for instance, in the half-space $\mathbb{R}_{+}^{1+q}$ with boundary $\mathbb{R}^{q}$, with given right hand sides $f, g$ and a so-called trace operator $T$ that represents the boundary conditions. $T$ may be given as a vector of operators of the form $u \rightarrow \mathrm{r}^{\prime} B u$, where $B$ is a differential operator and $\mathrm{r}^{\prime}$ the operator of restriction to the boundary. We then have the boundary symbol $\sigma_{\partial}(T)(y, \eta)$ consisting of the vector of operators $\mathrm{r}^{\prime} \sigma_{\psi}(B)\left(0, y, D_{r}, \eta\right): H^{s}\left(\mathbb{R}_{+}\right) \rightarrow \mathbb{C}$; in this case $\mathrm{r}^{\prime}$ is the restriction to $r=0$ and $s>\frac{1}{2}$ sufficiently large.

An example is $B \equiv 1$ and $T=\mathrm{r}^{\prime}$; this operator represents Dirichlet conditions. For $A=\Delta$, see Example 1.25, we obtain a family of isomorphisms.

$$
{ }^{\mathrm{t}}\left(\sigma_{\partial}(\Delta)(\eta) \sigma_{\partial}\left(\mathrm{r}^{\prime}\right)\right): H^{s}\left(\mathbb{R}_{+}\right) \rightarrow H^{s-2}\left(\mathbb{R}_{+}\right) \oplus \mathbb{C}
$$

for all $\eta \neq 0, s-2>-\frac{1}{2}$.

In general, if we have finitely many trace operators $T={ }^{\mathrm{t}}\left(T_{1}, \ldots, T_{j_{+}}\right)$, in elliptic boundary value problems, the role of $\sigma_{\partial}(T)(y, \eta)$ is to fill up the family of Fredholm operators (27) to a family of isomorphisms

$$
{ }^{\mathrm{t}}\left(\sigma_{\partial}(A)(y, \eta) \quad \sigma_{\partial}(T)(y, \eta)\right): H^{s}\left(\mathbb{R}_{+}\right) \rightarrow H^{s-\mu}\left(\mathbb{R}_{+}\right) \oplus \mathbb{C}^{j_{+}}
$$

for $\eta \neq 0$. If we want to complete the calculus by passing to parametrices of operators $\mathcal{A}={ }^{\mathrm{t}}\left(\begin{array}{ll}A & T\end{array}\right)$, we have to invert such isomorphisms and to find associated operators $\mathcal{P}:=\left(\begin{array}{ll}P & K\end{array}\right)$, now being of row matrix from and with pseudo-differential operators $P$ with the 'right' behaviour at the boundary. This is possible within the calculus of boundary value problems for operators with the so-called transmission property at the boundary, see [6] or several monographs, e.g., [63], [25], [75], or [31]. The operator $K$ is called a potential operator.

In this calculus we can compose operator block matrices as soon as the image of the first factor fits to the range of the second one. In addition it turns out that, in order to fill up the boundary symbol of such a pseudodifferential operator to a family of isomorphisms, in general we need trace and potential entries at the same time. This gives rise to $2 \times 2$ block matrices of 'boundary value problems'

$$
\mathcal{A}=\left(\begin{array}{ll}
A & K \\
T & Q
\end{array}\right)
$$

with a principal symbolic tuple $\sigma(\mathcal{A})=\left(\sigma_{\psi}(\mathcal{A}), \sigma_{\partial}(\mathcal{A})\right)$.

Before we return to the edge case we want to rephrase the Sobolev spaces occurring in (26) as edge spaces. 
Definition 1.26 [71] Let $H$ be a Hilbert space with group action $\kappa=\left\{\kappa_{\lambda}\right\}_{\lambda \in \mathbb{R}_{+}}$. Then the abstract edge space $\mathcal{W}^{s}\left(\mathbb{R}^{q}, H\right)$ of smoothness $s \in \mathbb{R}$ is defined to be the completion of $\mathcal{S}\left(\mathbb{R}^{q}, H\right)$ with respect to the norm

$$
\|u\|_{\mathcal{W}^{s}\left(\mathbb{R}^{q}, H\right)}=\left\{\int\langle\eta\rangle^{2 s}\left\|\kappa_{\langle\eta\rangle}^{-1} \hat{u}(\eta)\right\|_{H}^{2} \mathrm{~d} \eta\right\}^{1 / 2}
$$

(with $\hat{u}=F u$ being the Fourier transform in $\mathbb{R}^{q}$ and $\kappa_{\lambda}$ acting on the values of $\hat{u}(\eta)$ in $H)$.

Useful functional analytic results on these so-called abstract edge spaces may be found in [72], [75], [29], [31]. Apart from our calculus on singular manifolds, edge spaces in several variants have been also applied in [2] and [15].

Example 1.27 For $H=H^{s}\left(\mathbb{R}_{+}\right)$with $\left(\kappa_{\lambda} u\right)(r)=\lambda^{1 / 2} u(\lambda r), \lambda \in \mathbb{R}_{+}$, we have

$$
H^{s}\left(\mathbb{R}_{+}^{1+q}\right)=\mathcal{W}^{s}\left(\mathbb{R}^{q}, H^{s}\left(\mathbb{R}_{+}\right)\right)
$$

Remark 1.28 Given a Fréchet space $E$ written as a projective limit of Hilbert spaces $E^{j}, j \in \mathbb{N}$, with continuous embeddings $\ldots \hookrightarrow E^{j+1} \hookrightarrow E^{j} \hookrightarrow \ldots \hookrightarrow$ $E^{0}$, we say that $E$ is endowed with a group action $\kappa$, if $\kappa$ is a group action on $E^{0}$ and $\left.\kappa\right|_{E^{j}}$ a group action on $E^{j}$ for every $j$. In that case we can form the spaces $\mathcal{W}^{s}\left(\mathbb{R}^{q}, E^{j}\right)$, and we set

$$
\mathcal{W}^{s}\left(\mathbb{R}^{q}, E\right):=\lim _{j \in \mathbb{N}} \mathcal{W}^{s}\left(\mathbb{R}^{q}, E^{j}\right)
$$

Example 1.29 (i) Let $H:=K^{s, \gamma}\left(X^{\Delta}\right)$ be endowed with the group action as in Remark 1.8 for $g=s-\gamma$, see also the notation (6). We then have the spaces

$$
\mathcal{W}^{s}\left(\mathbb{R}^{q}, K^{s, \gamma}\left(X^{\Delta}\right)\right)
$$

for every $s, \gamma \in \mathbb{R}$.

(ii) Let $E=K_{P}^{s, \gamma}\left(X^{\Delta}\right)$ for a discrete or continuous asymptotic type $P$, written as a projective limit of Hilbert spaces with the group action as in (i) (which is always possible). Then we obtain $\mathcal{W}^{s}\left(\mathbb{R}^{q}, K_{P}^{s, \gamma}\left(X^{\Delta}\right)\right.$ ) which is an edge space with asymptotics.

Definition $1.30 \quad$ (i) Let $M$ be a manifold with edge $Y$. Then $H_{[\mathrm{loc})}^{s, \gamma}(M)$ denotes the subspace of all $u \in H_{\mathrm{loc}}^{s}(M \backslash Y)$ such that the push forward of $\left.u\right|_{V \backslash Y}$ under $V \backslash Y \rightarrow X^{\wedge} \times \mathbb{R}^{q}$, multiplied by any $\varphi \in C_{0}^{\infty}\left(\mathbb{R}_{+} \times\right.$ $\left.\mathbb{R}^{q}\right)$, belongs to $\mathcal{W}^{s}\left(\mathbb{R}^{q}, K^{s, \gamma}\left(X^{\Delta}\right)\right)$. This is required for every $V$ as in Remark 1.17 , with $\mathbb{R}^{q}$ instead of $\Omega$, see the formula (17). 
(ii) Replacing in the latter condition $K^{s, \gamma}\left(X^{\Delta}\right)$ by $K_{P}^{s, \gamma}\left(X^{\Delta}\right)$ for a (continuous) asymptotic type $P$ (satisfying the shadow condition) we obtain the space $H_{[\mathrm{loc}), P}^{s, \gamma}(M)$. Similarly, $H_{[\mathrm{comp})}^{s, \gamma}(M)$ and $H_{[\mathrm{comp}), P}^{s, \gamma}(M)$ are defined to be the subspaces of the corresponding '[loc)'-versions of elements with compact support.

If $M$ itself is compact, we omit '[comp)' and '[loc)' and simply write

$$
H^{s, \gamma}(M) \text { and } H_{P}^{s, \gamma}(M),
$$

respectively.

The notation also makes sense for the case $\operatorname{dim} Y=0$, i.e., conical singularities.

Proposition 1.31 An operator $A \in \operatorname{Diff}_{\mathrm{deg}}^{\mu}(M), M \in \mathfrak{M}_{1}$ compact, induces continuous operators

$$
A: H^{s, \gamma}(M) \rightarrow H^{s-\mu, \gamma-\mu}(M)
$$

and

$$
A: H_{P}^{s, \gamma}(M) \rightarrow H_{Q}^{s-\mu, \gamma-\mu}(M)
$$

for all $s, \gamma \in \mathbb{R}$ and every asymptotic type $P$ with some resulting asymptotic type $Q$.

Assuming now that $A \in \operatorname{Diff}_{\operatorname{deg}}^{\mu}(M)$ is $\sigma_{\psi}$-elliptic in the sense of Definition 1.21 , it is a natural question, whether the operator (29) can be seen as the 'upper left corner' of a Fredholm operator

$$
\mathcal{A}=\left(\begin{array}{ll}
A & K \\
T & Q
\end{array}\right): \underset{H^{s}\left(Y, J_{-}\right)}{H^{s, \gamma}(M)} \rightarrow \underset{H^{s-\mu}\left(Y, J_{+}\right)}{H^{s-\mu, \gamma-\mu}(M)}
$$

for suitable $J_{ \pm} \in \operatorname{Vect}(Y)$ (Vect(.) means the set of all smooth complex vector bundles over the manifold in parentheses). In simplest cases we may imagine trivial bundles $J_{ \pm}=Y \times \mathbb{C}^{j_{ \pm}}$for suitable $j_{ \pm} \in \mathbb{N}$, also denoted by $\mathbb{C}^{j_{ \pm}}$.

Answers are given within the pseudo-differential edge algebra, first developed in this generality in [71] and then more and more completed in several papers and monographs, see the references in the introduction and also the comments in [80]. Note, in particular, that the calculus of [71] contains information from [64] which is a variant of the edge calculus when the base of 
the model cone is of dimension 0 (i.e., a calculus of boundary value problems without the transmission property at the boundary) and from [65], [66], [68], [67]. A later investigation of Mazzeo [49] on edge-degenerate operators employed rather different techniques. Trace and potential operators on the edge are not considered there.

In Section 3 below we will develop more details on the structure of cone and edge algebras.

\subsection{Higher singularities}

We now turn to categories $\mathfrak{M}_{k}$ of manifolds of singularity order $k \geq 1$. The definition will be inductive. The categories $\mathfrak{M}_{0}$ and $\mathfrak{M}_{1}$ are introduced in Section 1.1 and 1.2. By induction assumption, in future referred to as (IA), we employ the categories $\mathfrak{M}_{l}$ for all $0 \leq l \leq k-1, k \geq 2$, and then pass from $k-1$ to $k$. The information feeded in by (IA) is that

$$
X \in \mathfrak{M}_{l} \Rightarrow X \times \Omega \in \mathfrak{M}_{l}
$$

for any open $C^{\infty}$ manifold $\Omega$. This allows us to form $X^{\wedge}=\mathbb{R}_{+} \times X \in \mathfrak{M}_{l}$, and we can define (locally trivial)

$$
X \text { - bundles or } X^{\wedge} \text {-bundles. }
$$

General notions and constructions on fibre bundle over a $C^{\infty}$ manifold can easily be adapted to the case of such fibres, by systematically using that by (IA) we know not only the objects in $\mathfrak{M}_{l}$ but also isomorphisms and morphisms in that category.

Let $\mathfrak{M}_{l}^{\wedge}\left(\mathbb{R} \times \mathfrak{M}_{l}\right)$ denote the subcategory of all spaces of the form $X^{\wedge}(\mathbb{R} \times$ $X)$ for $X \in \mathfrak{M}_{l}$. Moreover, define the category

$$
\overline{\mathbb{R}}_{+} \times \mathfrak{M}:=\left\{\overline{\mathbb{R}}_{+} \times X: X \in \mathfrak{M}_{l}\right\}
$$

where an isomorphism (morphism)

$$
\overline{\mathbb{R}}_{+} \times X \rightarrow \overline{\mathbb{R}}_{+} \times \tilde{X}
$$

in (32) is a homeomorphism (continuous map) such that there is an isomorphism (morphism)

$$
\mathbb{R} \times X \rightarrow \mathbb{R} \times \tilde{X}
$$

in $\mathbb{R} \times \mathfrak{M}_{l}$ which restricts to isomorphisms (morphisms) $\{0\} \times X \rightarrow\{0\} \times \widetilde{X}$ and $\mathbb{R}_{+} \times X \rightarrow \mathbb{R}_{+} \times X$ in $\mathfrak{M}_{l}$ and $\mathfrak{M}_{l}^{\wedge}$, respectively (clearly $\{0\} \times X$ is identified with $X$ ). 
We then define the category $\mathfrak{M}_{l}^{\Delta}$ of cones $X^{\Delta}=\left(\overline{\mathbb{R}}_{+} \times X\right) /(\{0\} \times X)$, where an isomorphism (morphism) $X^{\Delta} \rightarrow X^{\Delta}$ is the quotient map of some isomorphism (morphism) (33) in $\overline{\mathbb{R}}_{+} \times \mathfrak{M}_{l}$ with respect to the map $\{0\} \times X \rightarrow$ $\{0\} \times \widetilde{X}$ between the corresponding subspaces.

This allows us to form (locally trivial) $X^{\Delta_{\text {-bundles }}} L$ and $\left(\overline{\mathbb{R}}_{+} \times X\right)$ bundles $\mathbb{L}$ over a $C^{\infty}$ manifold $Y$. Such an $\mathbb{L}$ contains an $X$-bundle $\mathbb{L}_{\text {sing }}$ and an $X^{\wedge}$-bundle $\mathbb{L}_{\text {reg }}$ as subbundles, where

$$
\mathbb{L}=\mathbb{L}_{\text {sing }} \cup \mathbb{L}_{\text {reg }}
$$

(disjoint union), and there is a map $\pi: \mathbb{L} \rightarrow L$ to an $X^{\Delta}$-bundle over $Y$ by fibrewise applying the quotient map $\overline{\mathbb{R}}_{+} \times X \rightarrow\left(\overline{\mathbb{R}}_{+} \times X\right) /(\{0\} \times X)$. For instance, if $\mathbb{L}=\overline{\mathbb{R}}_{+} \times X \times \Omega$, then $\mathbb{L}_{\text {sing }}=\{0\} \times X \times \Omega, \mathbb{L}_{\mathrm{reg}}=\mathbb{R}_{+} \times X \times \Omega$, and $L=X^{\Delta} \times \Omega$.

Remark 1.32 By (IA) we know that $X$-, $X^{\wedge}$ - and $\mathbb{R} \times X$-bundles over a smooth manifold $Y$ for $X \in \mathfrak{M}_{l}, 0 \leq l \leq k-1$, also belong to $\mathfrak{M}_{l}$. However, our $X^{\Delta}$-bundles $L$ over $Y$ will already be of singularity order $k$ when $X \in \mathfrak{M}_{k-1}$. This is a part of Definition 1.33 below.

A simple consideration then shows that $X$ - and $X^{\wedge}$-bundles as well as $(\mathbb{R} \times X)$-bundles for $X \in \mathfrak{M}_{l}, 0 \leq l \leq k-1$, are objects in corresponding categories with isomorphisms (morphisms) referring to the bundle structure, including the compatibility with the respective bundle projections. This gives rise also to a category of $X^{\Delta}$-bundles when we employ the quotient spaces fibrewise and the above-mentioned (iso)morphisms between them.

By construction, $\mathbb{L}$ as an $\overline{\mathbb{R}}_{+} \times X$-bundle, is a subbundle of an $\mathbb{R} \times X$ bundle that we denote by $2 \mathbb{L}$ (the double).

Definition 1.33 A topological space $M$ is said to be a manifold of singularity order $k \geq 1$, written $M \in \mathfrak{M}_{k}$, if

(i) there is chosen a subspace $Y \subset M, Y \in \mathfrak{M}_{0}$, such that $M \backslash Y \in \mathfrak{M}_{k-1}$;

(ii) $Y$ has a neighbourhood $U$ in $M$ which has the structure of a (locally trivial) cone bundle $L$ over $Y$ with fibre $X^{\Delta}$ for some $X \in \mathfrak{M}_{k-1}$, i.e., $U \cong L$ (in the sense of isomorphy of such cone bundles).

We call $Y$ the minimal stratum of $M$.

Denoting the manifold $Y$ in Definition 1.33 for the moment by $Y^{k}$, for $M \backslash Y^{k} \in \mathfrak{M}_{k-1}$ we have again a minimal stratum $Y^{k-1} \in \mathfrak{M}_{0}$ such that $\left(M \backslash Y^{k}\right) \backslash Y^{k-1} \in \mathfrak{M}_{k-2}$, and so on. In this way we obtain a sequence of $C^{\infty}$ manifolds $Y^{k}, Y^{k-1}, \ldots, Y^{1}, Y^{0}$, such that $M$ can be written as a disjoint union (1). Let us call $Y^{0}$ the maximal stratum of $M$ and $\operatorname{set} \operatorname{dim} M=\operatorname{dim} Y^{0}$. 
Remark 1.34 With every $M \in \mathfrak{M}_{k}$ we can associate its stretched manifold $\mathbb{M}$ by invariantly attaching the $X$-bundle $\mathbb{U}_{\text {sing }}$ to $M \backslash Y^{k}$; here, similarly as (34), for $U \cong L$ we form $\mathbb{U} \cong \mathbb{L}$ in the sense of $\overline{\mathbb{R}}_{+} \times X$-bundles, and $\mathbb{U}=\mathbb{U}_{\text {sing }} \cup \mathbb{U}_{\text {reg. }}$. We then write

$$
\mathbb{M}=\mathbb{M}_{\text {sing }} \cup \mathbb{M}_{\text {reg }}
$$

for $\mathbb{M}_{\text {sing }}:=\mathbb{U}_{\text {sing }}$ and $\mathbb{M}_{\text {reg }}:=\mathbb{M} \backslash \mathbb{M}_{\text {sing }} \cong M \backslash Y^{k}$. From this construction we obtain a continuous map $\pi: \mathbb{M} \rightarrow M$ such that $\left.\pi\right|_{\mathbb{M}_{\text {sing }}}: \mathbb{M}_{\text {sing }} \rightarrow Y^{k}$ is the bundle projection of the respective $X$-bundle over $Y^{k}$ and $\left.\pi\right|_{\mathbb{M}_{\mathrm{reg}}}: \mathbb{M}_{\mathrm{reg}} \rightarrow$ $M \backslash Y^{k}$ an isomorphism in $\mathfrak{M}_{k-1}$. By gluing together two copies of $\mathbb{M}$ along $\mathbb{U}_{\text {sing }}$ we obtain the double $2 \mathbb{M}$ of $\mathbb{M}$; then $2 \mathbb{M} \in \mathfrak{M}_{k-1}$.

Remark 1.35 We interpret $\mathfrak{M}_{k}$ as a category, where the isomorphisms (morphisms) $\beta: M \rightarrow \widetilde{M}$ are homeomorphisms (continuous maps) which restrict to isomorphisms (morphisms) $M \backslash Y^{k} \rightarrow M \backslash \widetilde{Y}^{k}$ in $\mathfrak{M}_{k-1}$ and diffeomorphisms (differentiable maps) $Y^{k} \rightarrow \widetilde{Y}^{k}$, such that there is an isomorphism (morphism) $2 \mathbb{M} \rightarrow 2 \widetilde{\mathbb{M}}$ in $\mathfrak{M}_{k-1}$ of the doubles of the associated stretched spaces, such that its restriction $b: \mathbb{M} \rightarrow \widetilde{\mathbb{M}}$ satisfies the condition $\beta \circ \pi=\tilde{\pi} \circ b($ where $\tilde{\pi}: \widetilde{\mathbb{M}} \rightarrow \widetilde{M}$ is of analogous meaning as the abovementioned $\pi: \mathbb{M} \rightarrow M$ ), and $b$ restricts to a bundle isomorphism (morphism) $\mathbb{M}_{\text {sing }} \rightarrow \widetilde{\mathbb{M}}_{\text {sing }}$ of the $X$-bundle over $Y^{k}$ to the respective $\widetilde{X}$-bundle over $\widetilde{Y}^{k}$.

Now the constructions at the beginning of this section in connection with the categories $\mathfrak{M}_{l}^{\wedge}, \mathbb{R} \times \mathfrak{M}_{l}, \overline{\mathbb{R}}_{+} \times \mathfrak{M}_{l}, \mathfrak{M}_{l}^{\Delta}$, and bundles $L, \mathbb{L}, 2 \mathbb{L}$ etc, can easily be performed up to $l=k$. This allows us to formulate an analogue of Definition 1.33 for $k+1$ instead of $k$.

Theorem 1.36 $M \in \mathfrak{M}_{k}, N \in \mathfrak{M}_{l}$ implies $M \times N \in \mathfrak{M}_{k+l}$ for every $k, l \in \mathbb{N}$.

We now turn to spaces of differential operators on $\mathfrak{M}_{k}$ and their symbolic structures.

By (IA) we have the spaces $\operatorname{Diff}_{\text {deg }}^{\mu}(X)$ for every $X \in \mathfrak{M}_{l}$ for $0 \leq l \leq$ $k-1$, and those spaces are Fréchet in a natural way. Moreover, we have the principal symbolic hierarchy

$$
\sigma(A):=\left(\sigma_{j}(A)\right)_{0 \leq j \leq l}
$$

for $A \in \operatorname{Diff}_{\text {deg }}^{\mu}(X), X \in \mathfrak{M}_{l}$. Furthermore, by (IA) we know that an isomorphism

$$
\varphi: X \rightarrow \tilde{X}
$$

in $\mathfrak{M}_{l}$ induces an isomorphism

$$
\varphi_{*}: \operatorname{Diff}_{\mathrm{deg}}^{\mu}(X) \rightarrow \operatorname{Diff}_{\mathrm{deg}}^{\mu}(\tilde{X})
$$

of the corresponding spaces of operators. 
Definition 1.37 Let $M \in \mathfrak{M}_{k}, k \geq 1$, and $Y^{k} \subset M$ the minimal stratum of $M$. Then an operator $A \in \operatorname{Diff}_{\mathrm{deg}}^{\mu}\left(M \backslash Y^{k}\right)$ (the latter space known by (IA)) is said to belong to $\operatorname{Diff}_{\mathrm{deg}}^{\mu}(M)$, if

(i) in the case $\operatorname{dim} Y^{k}=0$ the operator has close to $Y^{k}$ in the splitting of variables $(t, x) \in \mathbb{R}_{+} \times X_{k-1}, X_{k-1} \in \mathfrak{M}_{k-1}$, the form

$$
A=t^{-\mu} \sum_{j=0}^{\mu} a_{j}(t)\left(-t \frac{\partial}{\partial t}\right)^{j}
$$

with coefficients $a_{j} \in C^{\infty}\left(\overline{\mathbb{R}}_{+}, \operatorname{Diff}_{\mathrm{deg}}^{\mu-j}\left(X_{k-1}\right)\right)$;

(ii) in the case $\operatorname{dim} Y^{k}=: q_{k}>0$ the operator has close to $Y^{k}$ in the splitting of variables $(t, x, z) \in \mathbb{R}_{+} \times X \times \Omega_{k}, X_{k-1} \in \mathfrak{M}_{k-1}, \Omega_{k} \subseteq \mathbb{R}^{q_{k}}$ open, the form

$$
A=t^{-\mu} \sum_{j+|\alpha| \leq \mu} a_{j \alpha}(t, z)\left(-t \frac{\partial}{\partial t}\right)^{j}\left(t D_{z}\right)^{\alpha}
$$

with coefficients $a_{j \alpha} \in C^{\infty}\left(\overline{\mathbb{R}}_{+} \times \Omega_{k}\right.$, Diff $\left.\operatorname{deg}^{\mu-(j+|\alpha|)}\left(X_{k-1}\right)\right)$.

Remark 1.38 Applying Definition 1.33 for $k \geq 2$ once again to $X \in \mathfrak{M}_{k-1}$, i.e., to a minimal stratum $Y \subset X, Y \in \mathfrak{M}_{0}$, and taking into account that $X \backslash Y \in \mathfrak{M}_{k-2}$, we see that every $y \in Y$ has a neighbourhood $V$ in $X$, where $V \backslash Y$ admits a local splitting of variables into $(r, x, y) \in \mathbb{R}_{+} \times X_{k-2} \times \Omega_{k-1}$, $\Omega_{k-1} \subseteq \mathbb{R}^{q_{k-1}}$ open, $X_{k-2} \in \mathfrak{M}_{k-2}$. Then (35) takes the local form

$$
A=t^{-\mu} r^{-\mu} \sum_{j+|\alpha|+l+|\beta| \leq \mu} a_{j \alpha, l \beta}(t, r, y, z)\left(-r \frac{\partial}{\partial r}\right)^{l}\left(r D_{y}\right)^{\beta}\left(-r t \frac{\partial}{\partial t}\right)^{j}\left(r t D_{z}\right)^{\alpha}
$$

with coefficients

$$
a_{j \alpha, l \beta} \in C^{\infty}\left(\overline{\mathbb{R}}_{+} \times \overline{\mathbb{R}}_{+} \times \Omega_{k-1} \times \Omega_{k}, \operatorname{Diff}_{\mathrm{deg}}^{\mu-(j+|\alpha|+l+|\beta|}\left(X_{k-2}\right)\right) .
$$

Example 1.39 Let $X \in \mathfrak{M}_{0}$ be equipped with a Riemannian metric $g_{X}$. Then, as noted in the introduction, the Laplace-Beltrami operator belonging to the wedge metric

$$
d r^{2}+r^{2} g_{X}+d y^{2}
$$

on $W:=\mathbb{R}_{+} \times X \times \Omega \ni(r, x, y), \Omega_{1} \subseteq \mathbb{R}^{q_{1}}$ open, is edge-degenerate and of order 2. Taking $W$ as the cone of another wedge $V:=\mathbb{R}_{+} \times W \times \Omega_{2} \ni$ $(t,(r, x, y), z)$ for $\Omega_{2} \subseteq \mathbb{R}^{q_{2}}$ open, we can form the corner metric

$$
d t^{2}+t^{2}\left(d r^{2}+r^{2} g_{X}+d y^{2}\right)+d z^{2} .
$$


The associated Laplace-Beltrami operator is then of the form (35) for $\mu=2$. The process of iteratively forming higher wedge metrics can be continued, and then, if $k$ is the corresponding singularity order, the associated LaplaceBeltrami operator belongs to Diff 2 deg over that higher wedge.

Definition 1.40 Let $M \in \mathfrak{M}_{k}, Y^{k} \subset M$ its minimal stratum (see Definition 1.33), and let $A \in \operatorname{Diff}_{\operatorname{deg}}^{\mu}(M)$. We then set

$$
\sigma(A):=\left(\sigma\left(\left.A\right|_{M \backslash Y^{k}}\right), \sigma_{k}(A)\right),
$$

where $\sigma\left(\left.A\right|_{M \backslash Z}\right):=\left(\sigma_{0}(A), \ldots, \sigma_{k-1}(A)\right)$ is known by $(I A)$ from $M \backslash Y^{k} \in$ $\mathfrak{M}_{k-1}$, and

$$
\sigma_{k}(A)(w):=\sum_{j=0}^{\mu} a_{j}(0) w^{j} \text { for } \operatorname{dim} Y^{k}=0
$$

as a w-depending family of operators in $\operatorname{Diff}_{\mathrm{deg}}^{\mu}(X), w \in \mathbb{C}$,

$$
\sigma_{k}(A)(z, \zeta):=t^{-\mu} \sum_{j+|\alpha| \leq \mu} a_{j \alpha}(0, z)\left(-t \frac{\partial}{\partial t}\right)^{j}(t \zeta)^{\alpha} \text { for } \operatorname{dim} Y^{k}>0
$$

as a $(z, \zeta)$-depending family of operators in $\operatorname{Diff}_{\mathrm{deg}}^{\mu}\left(X^{\wedge}\right),(z, \zeta) \in \Omega_{k} \times\left(\mathbb{R}^{q_{k}} \backslash\right.$ $\{0\})$.

Theorem 1.41 $A \in \operatorname{Diff}_{\mathrm{deg}}^{\mu}(M), B \in \operatorname{Diff}_{\mathrm{deg}}^{\nu}(M)$ implies $A B \in \operatorname{Diff}_{\mathrm{deg}}^{\mu+\nu}(M)$, and we have $\sigma(A B)=\sigma(A) \sigma(B)$, with componentwise composition of an the right hand side (and the rule $\sigma_{k}(A B)=\left(T^{\nu} \sigma_{k}(A)\right) \sigma(B)$ when the dimension of the minimal stratum is zero; recall that $\left.\left(T^{\beta} f\right)(w)=f(w+\beta)\right)$.

The proof is simple when we employ (IA) for the operators realised on $M \backslash Y^{k} \in \mathfrak{M}_{k-1}$ and then compute the rule for the $k$-th symbolic component directly.

Remark 1.42 By repeatedly applying Definition 1.33 locally near neighbourhoods of minimal strata, first near any point of $Y$ (where $Y$ denotes again the minimal stratum of $M)$ in a trivialisation of $\mathbb{U}_{\text {reg }}$ over a coordinate neighbourhood $\Omega_{k}$ on $Y$, i.e., $\left.\mathbb{U}_{\text {reg }}\right|_{\Omega_{k}} \cong \mathbb{R}_{+} \times X_{k-1} \times \Omega_{k} \ni\left(r_{k}, x_{k-1}, y_{k}\right)$ (here in modified notation compared to the one before), then similarly near the minimal stratum of $X \in \mathfrak{M}_{k-1}$ in a local splitting of variables, $\left(r_{k-1}, x_{k-2}, y_{k-1}\right) \in$ $\mathbb{R}_{+} \times X_{k-2} \times \Omega_{k-1}$, and so on, we can introduce locally close to $Y$ on $M$ coordinates

$$
\left(r_{1}, \ldots, r_{k}, x, y_{1}, \ldots, y_{k}\right) \in\left(\mathbb{R}_{+}\right)^{k} \times \Sigma \times \prod_{j=1}^{k} \Omega_{j},
$$


$\Omega_{j} \subseteq \mathbb{R}^{q_{j}}$ open, $\Sigma \subseteq \mathbb{R}^{n}$ open, $y_{j} \in \Omega_{j}, x \in \Sigma$, where an operator $A \in$ $\operatorname{Diff}_{\mathrm{deg}}^{\mu}(M)$ has the form $A=r_{1}^{-\mu} \ldots r_{k}^{-\mu} A_{0}$, and $A_{0}$ is a polynomial in the vector fields

$$
\begin{aligned}
& r_{1} \frac{\partial}{\partial r_{1}}, r_{1} r_{2} \frac{\partial}{\partial r_{2}}, \ldots, r_{1} \ldots r_{2} \frac{\partial}{\partial r_{k}}, \frac{\partial}{\partial x_{1}}, \ldots, \frac{\partial}{\partial x_{n}}, r_{1} \frac{\partial}{\partial y_{1, i}}, i=1, \ldots, q_{1}, \\
& \quad r_{1} r_{2} \frac{\partial}{\partial y_{2, i}}, i=1, \ldots, q_{2}, \ldots, r_{1} \ldots r_{k} \frac{\partial}{\partial y_{k, i}}, i=1, \ldots q_{k},
\end{aligned}
$$

with coefficients in $C^{\infty}\left(\left(\overline{\mathbb{R}}_{+}\right)^{k} \times \Sigma \times \prod_{j=1}^{k} \Omega_{j}\right)$.

Remark 1.43 We do not study here operators of 'multi-Fuchs type', defined in terms of vector fields $r_{j} \frac{\partial}{\partial r_{j}}$, see [60], [59], [57], [58], and the references there. The resulting calculus is simpler than ours.

More generally, if we are given a space of 'typical' differential operators on the maximal stratum $Y^{0}$ of a corner manifold $M$ which are polynomials in vector fields of a prescribed 'degenerate' behaviour at $M \backslash Y^{0}$ (together with the coefficients), it may be an interesting task to establish a pseudodifferential calculus that extends the space of those differential operators to an algebra, closed under the construction of parametrices of elliptic elements (whatever 'ellipticity' means). In any case the result will depend on the vector fields in a specific way. What we do in the present paper is to treat operators in $\operatorname{Diff}_{\mathrm{deg}}^{\mu}(M)$ in the sense of Definition 1.37, cf. also Remark 1.42. 


\section{Pseudo-differential operators with param- eters and conical exits to infinity}

\subsection{Classical operators with parameters}

Standard material on pseudo-differential operators may be found in any textbook on this topic, see, for instance, Kumano-go [41], or Treves [94]. In the first part of this section we recall a few facts, mainly in order to fix our notation.

Parametrices of elliptic differential operators on a $C^{\infty}$ manifold $X$ exist in the class of classical pseudo-differential operators of opposite order. In local variables $x \in \Sigma, \Sigma \subseteq \mathbb{R}^{n}$ open, with the covariables $\xi \in \mathbb{R}^{n}$, the pseudodifferential operators of order $\mu \in \mathbb{R}$ are given as

$$
A u(x)=\operatorname{Op}(a) u(x)+C u(x)
$$

where $a(x, \xi)$ is an amplitude function (or symbol) in $C^{\infty}\left(\Sigma \times \mathbb{R}^{n}\right)$, and $C$ an operator with kernel in $C^{\infty}(\Sigma \times \Sigma)$. Concerning $a(x, \xi)$ we ask the symbolic estimates

$$
\sup _{x \in K}\left|D_{x}^{\alpha} D_{\xi}^{\beta} a(x, \xi)\right| \leq c\langle\xi\rangle^{\mu-|\beta|}
$$

for all $\xi \in \mathbb{R}^{n}$, multi-indices $\alpha, \beta$ and compact subsets $K \subset \Sigma$; here $\langle\xi\rangle:=$ $\left(1+|\xi|^{2}\right)^{1 / 2}, c=c(\alpha, \beta, K)>0$.

The corresponding space of such symbols is denoted here by $S^{\mu}\left(\Sigma \times \mathbb{R}^{n}\right)$ (and the subspace of $x$-independent elements by $S^{\mu}\left(\mathbb{R}^{n}\right)$ ). By admitting the dimension of $\xi$-variables independent of the dimension of $x$ we can also define spaces $S^{\mu}\left(\Sigma \times \mathbb{R}_{\xi, \lambda}^{n+l}\right)$, where $(\xi, \lambda) \in \mathbb{R}^{n+l}$ plays the role of covariables in the symbolic estimates.

The notation $\mathrm{Op}(a) u$ in (39) (also written $\left.\mathrm{Op}_{x}(a) u\right)$ means $\iint e^{i\left(x-x^{\prime}\right) \xi} a(x, \xi)$ $u\left(x^{\prime}\right) d x d \xi, d \xi=(2 \pi)^{-n} d \xi$, interpreted in the well-known oscillatory integral set-up. Globally on $X$ we define $L^{\mu}(X)$, the space of pseudo-differential operators of order $\mu$ as the set of of all $A_{0}+C$ where $A_{0}$ is an arbitrary locally finite sum of operators the form $\varphi\left(\chi^{-1}\right)_{*} \operatorname{Op}(a) \psi$, for any $\varphi, \psi \in C_{0}^{\infty}(U)$, and any chart $\chi: U \rightarrow \Sigma$ on $X$, where $\left(\chi^{-1}\right)_{*}$ means the push forward of operators from $\Sigma$ to $U$ under $\chi^{-1}$, and $C$ a globally smoothing operator. The space $L^{-\infty}(X)$ is identified with $C^{\infty}(X \times X)$ via a Riemannian metric on $X$. Let $L_{\mathrm{cl}}^{\mu}(X)$ denote the subspace of all elements of $L^{\mu}(X)$ where the symbols $a(x, \xi)$ are classical, i.e., locally in $(x, \xi) \in \Sigma \times \mathbb{R}^{n}$ there exist $C^{\infty}$ functions $a_{(\mu-j)}(x, \xi)$ in $\Sigma \times\left(\mathbb{R}^{n} \backslash\{0\}\right), j \in \mathbb{N}$, such that $a_{(\mu-j)}(x, \lambda \xi)=\lambda^{\mu-j} a_{(\mu-j)}(x, \xi)$ for $\lambda \in \mathbb{R}_{+}$and $a(x, \xi)-\chi(\xi) \sum_{j=0}^{N} a_{(\mu-j)}(x, \xi) \in S^{\mu-(N+1)}\left(\Sigma \times \mathbb{R}^{n}\right)$ for any excision function $\chi(\xi)$ (i.e., $\chi \in C^{\infty}\left(\mathbb{R}^{n}\right), \chi(\xi)=0$ for $|\xi|<c_{0}, \chi(\xi)=1$ 
for $|\xi|>c_{1}$ for some constants $0<c_{0}<c_{1}$ ). The space of those symbols is denoted by $S_{\mathrm{cl}}^{\mu}\left(\Sigma \times \mathbb{R}^{n}\right)$ (and the subspace of $x$-independent elements by $S_{\mathrm{cl}}^{\mu}\left(\mathbb{R}^{n}\right)$; later on we employ this space with its nuclear Fréchet topology). In a similar manner we define $S_{\mathrm{cl}}^{\mu}\left(\Sigma \times \mathbb{R}_{\xi, \lambda}^{n+l}\right)$. If a consideration is valid both in the classical and the general case; we often write subscript '(cl)'.

Setting $L^{-\infty}\left(X ; \mathbb{R}^{l}\right)=\mathcal{S}\left(\mathbb{R}^{l}, L^{-\infty}(X)\right)$ we can define the space $L_{(\mathrm{cl})}^{\mu}\left(X ; \mathbb{R}^{l}\right)$ of all parameter-dependent pseudo-differential operators on $X$ which are of the form $A_{0}(\lambda)+C(\lambda)$, where $A_{0}(\lambda)$ is defined similarly as $A_{0}$, now with symbols $a(x, \xi, \lambda) \in S_{(\mathrm{cl})}^{\mu}\left(\Sigma \times \mathbb{R}^{n+l}\right)$ and $C(\lambda) \in L^{-\infty}\left(X ; \mathbb{R}^{l}\right)$ where $A_{0}(\lambda)$ is defined in a similar manner as $A_{0}$, now with symbols $a(x, \xi, \lambda) \in S_{(\mathrm{cl})}^{\mu}(\Sigma \times$ $\left.\mathbb{R}^{n+l}\right)$, and $C(\lambda) \in L^{-\infty}\left(X ; \mathbb{R}^{l}\right)$.

Every $A \in L_{\mathrm{cl}}^{\mu}\left(X ; \mathbb{R}^{l}\right)$ has a parameter-dependent homogeneous principal symbol $\sigma_{\psi}(A)$, locally depending on the variables $(x, \xi, \lambda) \in \Sigma \times\left(\mathbb{R}^{n+l} \backslash\{0\}\right)$ and homogeneous in $(\xi, \lambda)$ of order $\mu$ and invariantly defined as a $C^{\infty}$ function on $T^{*} X \times \mathbb{R}^{l} \backslash 0$.

An $A \in L^{\mu}\left(X ; \mathbb{R}^{l}\right)$ is said to be properly supported, if the support $S_{\lambda}$ of the distributional kernel of $A(\lambda)$ in $\mathcal{D}^{\prime}(X \times X)$ has the property that the sets $S_{\lambda} \cap\left\{\left(x, x^{\prime}\right): x^{\prime} \in K^{\prime}\right\}$ and $S_{\lambda} \cap\left\{\left(x, x^{\prime}\right): x \in K\right\}$ are compact in $X \times X$ for arbitrary compact sets $K, K^{\prime}$ in $X$; this is required for all $\lambda \in \mathbb{R}^{l}$. It can be proved that every $A \in L^{\mu}\left(X ; \mathbb{R}^{l}\right)$ has a properly supported representative $A_{1}$ modulo some $L^{-\infty}\left(X ; \mathbb{R}^{l}\right)$, where the support of the kernel of $A_{1}(\lambda)$ is contained in a fixed proper subset of $X \times X$ for all $\lambda$.

For simplicity we now concentrate on classical operators and $X$ compact. $A \in L_{\mathrm{cl}}^{\mu}\left(X ; \mathbb{R}^{l}\right), B \in L_{\mathrm{cl}}^{\nu}\left(X ; \mathbb{R}^{l}\right)$ and $A$ or $B$ properly supported entails $A B \in L_{\mathrm{cl}}^{\mu+\nu}\left(X ; \mathbb{R}^{l}\right)$, and we have $\sigma_{\psi}(A B)=\sigma_{\psi}(A) \sigma_{\psi}(B)$.

Definition 2.1 An operator $A \in L_{\mathrm{cl}}^{\mu}\left(X ; \mathbb{R}^{l}\right)$ is said to be parameter-dependent elliptic, if $\sigma_{\psi}(A)(x, \xi, \lambda) \neq 0$ on $T^{*} X \times \mathbb{R}^{l} \backslash 0$ (for $l=0$ we simply talk about ellipticity).

Theorem 2.2 Let $A \in L_{\mathrm{cl}}^{\mu}\left(X ; \mathbb{R}^{l}\right)$ be (parameter-dependent) elliptic. Then there is a properly supported parametrix $P$ in the sense $1-P A, 1-A P \in$ $L^{-\infty}\left(X ; \mathbb{R}^{l}\right)$ (with 1 being the identity operator).

Theorem 2.3 Let $A \in L_{\mathrm{cl}}^{\mu}\left(X ; \mathbb{R}^{l}\right)$ be parameter-dependent elliptic, $X$ compact. Then the associated operators

$$
A(\lambda): H^{s}(X) \rightarrow H^{s-\mu}(X)
$$

are Fredholm for all $\lambda \in \mathbb{R}^{l}, s \in \mathbb{R}$. They are of index 0 when $l>0$; there is then a $C>0$ such that the operators (41) are isomorphisms for all $|\lambda| \geq C$. 
The following material belongs to the basics of the pseudo-differential cone and edge algebras, see [72].

Definition 2.4 Let $M_{\mathcal{O}}^{\mu}\left(X ; \mathbb{R}^{q}\right)$ for $\mu \in \mathbb{R}, q \in \mathbb{N}$, denote the space of all entire functions $h(w)$ in $\mathbb{C}$ with values in $L_{\mathrm{cl}}^{\mu}\left(X ; \mathbb{R}_{\eta}^{q}\right)$ such that $h(\beta+i \varrho, \eta) \in$ $L_{\mathrm{cl}}^{\mu}\left(X ; \mathbb{R}_{\varrho, \eta}^{1+q}\right)$ for every $\beta \in \mathbb{R}$, uniformly in compact $\beta$-intervals. For $q=0$ we simply write $M_{\mathcal{O}}^{\mu}(X)$.

Theorem 2.5 Let $f(w, \eta) \in L_{\mathrm{cl}}^{\mu}\left(X ; \Gamma_{\beta} \times \mathbb{R}^{q}\right)$ be a parameter-dependent family with parameter $(\operatorname{Im} w, \eta) \in \mathbb{R}^{1+q}, w \in \Gamma_{\beta}$, for some fixed $\beta \in \mathbb{R}$. Then there exists an $h(w, \eta) \in M_{\mathcal{O}}^{\mu}\left(X ; \mathbb{R}^{q}\right)$ such that

$$
\left.h(w, \eta)\right|_{\Gamma_{\beta} \times \mathbb{R}^{q}}-f(w, \eta) \in L^{-\infty}\left(X ; \Gamma_{\beta} \times \mathbb{R}^{q}\right) .
$$

Moreover, if $f(w, \eta)$ is parameter-dependent elliptic with parameter $(w, \eta) \in$ $\Gamma_{\beta} \times \mathbb{R}^{q}$, then $\left.h(w, \eta)\right|_{\Gamma_{\alpha} \times \mathbb{R}^{q}}$ is also parameter-dependent elliptic with parameter $(w, \eta) \in \Gamma_{\alpha} \times \mathbb{R}^{q}$, for every $\alpha \in \mathbb{R}$, uniformly in compact $\alpha$-intervals.

Let $X$ be compact. A set of triples

$$
R=\left\{\left(p_{j}, m_{j}, L_{j}\right)\right\}_{j \in \mathbb{Z}}
$$

where $p_{j} \in \mathbb{C}, m_{j} \in \mathbb{N}$ and finite-dimensional subspaces $L_{j} \subset L^{-\infty}(X)$ of operators of finite rank, is called a discrete asymptotic type of Mellin symbols, if the set $\pi_{\mathbb{C}} R=\left\{p_{j}\right\}_{j \in \mathbb{Z}}$ intersects every strip $\left\{c_{0} \leq \operatorname{Re} w \leq c_{1}\right\}$ in a finite set. Let $M_{R}^{-\infty}(X)$ for such an $R$ denote the set of all $f \in \mathcal{A}\left(\mathbb{C} \backslash \pi_{\mathbb{C}} R, L^{-\infty}(X)\right)$ which are meromorphic with poles at the points $p_{j}$ of multiplicity $m_{j}+1$ and Laurent coefficients at $\left(w-p_{j}\right)^{-(k+1)}$ belongong to $L_{j}(X), 0 \leq k \leq m_{j}$. We then set

$$
M_{R}^{\mu}(X):=M_{\mathcal{O}}^{\mu}(X)+M_{R}^{-\infty}(X) .
$$

Theorem 2.6 Let $X$ be compact, let $h \in M_{R}^{\mu}(X)$ be written as $h=h_{0}+l$ according to $(42), h_{0} \in M_{\mathcal{O}}^{\mu}(X), l \in M_{R}^{-\infty}(X)$, and let $\left.h_{0}\right|_{\Gamma_{\beta}}$ be parameterdependent elliptic with parameter $w \in \Gamma_{\beta}$, for some fixed $\beta \in \mathbb{R}$. Then there exists an $h^{-1} \in M_{S}^{\mu}(X)$ for a suitable discrete asymptotic type $S$ of Mellin symbols such that $h h^{-1}=h^{-1} h=1$ (in the sense of pointwise composition of meromorphic operator functions).

Remark 2.7 Functional analytic properties of meromorphic Fredholm functions are studied in [21] and [22], [23]. A beautiful factorisation result for meromorhic pseudo-differential families is obtained in [95]; concerning factorisations in a more 'abstract' set-up, see [24]. Meromorphic Fredholm families of corner operators are investigated in [78], [46], [26], see also [11] and [61] for the case of boundary value probems. 


\subsection{Corner-degenerate operators}

Let $M \in \mathfrak{M}_{1}$, and let $Y \subset M$ denote its minimal stratum. We then introduce $L_{\mathrm{deg}}^{\mu}(M)$ to be the subspace of all $A_{1}+C \in L_{\mathrm{cl}}^{\mu}(M \backslash Y)$ for any $C \in L^{-\infty}(M \backslash$ $Y$ ) such that locally near $Y$ in the splitting of variables $(r, x, y) \in \mathbb{R}_{+} \times X \times \Omega$, $X \in \mathfrak{M}_{0}, \Omega \subseteq \mathbb{R}^{q}$ open, the operator $A_{1}$ has the form

$$
r^{-\mu} \mathrm{Op}_{y} \mathrm{Op}_{r}(a)
$$

for an operator-valued amplitude function

$$
\begin{gathered}
a(r, y, \varrho, \eta):=\tilde{a}(r, y, r \varrho, r \eta), \\
\tilde{a}(r, y, \tilde{\varrho}, \tilde{\eta}) \in C^{\infty}\left(\overline{\mathbb{R}}_{+} \times \Omega, L_{\mathrm{cl}}^{\mu}\left(X ; \mathbb{R}_{\tilde{\varrho}, \tilde{\eta}}^{1+q}\right)\right),
\end{gathered}
$$

and $C \in L^{-\infty}(M \backslash Y)$. More generally, by

$$
L_{\mathrm{deg}}^{\mu}\left(M ; \mathbb{R}^{l}\right)
$$

we denote the subspace of all $A=A_{1}+C \in L_{\mathrm{cl}}^{\mu}\left(M \backslash Y ; \mathbb{R}^{l}\right)$ for arbitrary $C \in L^{-\infty}\left(M \backslash Y ; \mathbb{R}^{l}\right)$, where $A_{1}$ is locally near $Y$ of the form

$$
r^{-\mu} \mathrm{Op}_{y} \mathrm{Op}_{r}(a)(\lambda)
$$

for

$$
\begin{gathered}
a(r, y, \varrho, \eta, \lambda):=\tilde{a}(r, y, r \varrho, r \eta, r \lambda), \\
\tilde{a}(r, y, \tilde{\varrho}, \tilde{\eta}, \tilde{\lambda}) \in C^{\infty}\left(\overline{\mathbb{R}}_{+} \times \Omega, L_{(\mathrm{cl})}^{\mu}\left(X ; \mathbb{R}_{\tilde{\varrho}, \tilde{\eta}, \tilde{\lambda}}^{1+q_{1}+l}\right)\right) .
\end{gathered}
$$

By (IA) we know the spaces (43) for arbitrary $M \in \mathfrak{M}_{l}, \quad 0 \leq l \leq k-1$, including the fact that they are Fréchet spaces in a natural way.

Definition 2.8 Let $M \in \mathfrak{M}_{k}$ and $Y^{k}$ its minimal and $Y^{0}$ its maximal stratum. Then

$$
L_{\mathrm{deg}}^{\mu}\left(M ; \mathbb{R}^{l}\right),
$$

$\mu \in \mathbb{R}, l \in \mathbb{N}$, denotes the space of all operator families

$$
A=A_{1}+C
$$

for arbitrary $C \in L^{-\infty}\left(Y^{0} ; \mathbb{R}^{l}\right)$ where $A_{1}$ is locally near $Y^{k}$ in the splitting of variables $(r, x, y) \in \mathbb{R}_{+} \times X_{k-1} \times \Omega_{k}, X_{k-1} \in \mathfrak{M}_{k-1}, \Omega_{k} \subseteq \mathbb{R}^{q_{k}}$ open, of the form

$$
r^{-\mu} \mathrm{Op}_{y} \mathrm{Op}_{r}(a)(\lambda)
$$

where

$$
a(r, y, \varrho, \eta, \lambda):=\tilde{a}(r, y, r \varrho, r \eta, r \lambda)
$$

for an $\tilde{a}(r, y, \tilde{\varrho}, \tilde{\eta}, \tilde{\lambda}) \in C^{\infty}\left(\overline{\mathbb{R}}_{+} \times \Omega_{k}, L_{\mathrm{deg}}^{\mu}\left(M \backslash Y^{k} ; \mathbb{R}_{\tilde{\varrho}, \tilde{\eta}, \tilde{\lambda}}^{1+q_{k}+l}\right)\right)$.

The operators $A \in L_{\mathrm{deg}}^{\mu}\left(M ; \mathbb{R}^{l}\right)$ are called (parameter-dependent) cornerdegenerate. 
Remark 2.9 With the notation of Definition 2.8 we have

$$
L_{\mathrm{deg}}^{\mu}\left(M ; \mathbb{R}^{l}\right) \subset L_{\mathrm{cl}}^{\mu}\left(Y^{0} ; \mathbb{R}^{l}\right) .
$$

Moreover, every $A \in L_{\mathrm{cl}}^{\mu}\left(M ; \mathbb{R}^{l}\right)$ has a properly supported representative in

$$
L_{\mathrm{deg}}^{\mu}\left(M ; \mathbb{R}^{l}\right) \bmod L^{-\infty}\left(Y^{0} ; \mathbb{R}^{l}\right) .
$$

According to the relation (44) every $A \in L_{\text {deg }}^{\mu}\left(M ; \mathbb{R}^{l}\right)$ has a parameterdependent homogeneous principal symbol $\sigma_{\psi}(A) \in C^{\infty}\left(T^{*} Y^{0} \times \mathbb{R}^{l} \backslash 0\right)$. Locally in the splitting of variables $\left(r_{1}, \ldots, r_{k}, x, y_{1}, \ldots y_{k}\right)$ near $Y^{k}$ mentioned in Remark 2.9 and with the respective covariables (including the parameter) $\left(\varrho_{1}, \ldots, \varrho_{k}, \xi, \eta_{1}, \ldots \eta_{k}, \lambda\right)$ (and in modified notation with $(r, y)$ and $(\varrho, \eta)$ replaced by $\left(r_{k}, y_{k}\right)$ and $\left(\varrho_{k}, \eta_{k}\right)$, respectively) we have

$$
\begin{aligned}
\tilde{\sigma}_{\psi}(A) & (r, y, \varrho, \xi, \eta, \lambda):=r_{1}^{\mu} \ldots r_{k}^{\mu} \sigma_{\psi}\left(r, x, y, r_{1}^{-1} \varrho_{1}, \ldots,\right. \\
& \left.r_{1}^{-1} \ldots r_{k}^{-1} \varrho_{k}, \xi, r_{1}^{-1} \eta_{1}, \ldots, r_{1}^{-1} \ldots r_{k}^{-1} \eta_{k}, r_{1}^{-1} \ldots r_{k}^{-1} \lambda\right)
\end{aligned}
$$

for a function $\tilde{\sigma}_{\psi}(r, x, y, \tilde{\varrho}, \xi, \tilde{\eta}, \tilde{\lambda})$ which is smooth in $r \in\left(\overline{\mathbb{R}}_{+}\right)^{k}$ up to $r=$ $(0, \ldots, 0)$. We employed the abbreviation

$$
\begin{aligned}
& r=\left(r_{1}, \ldots, r_{k}\right), y=\left(y_{1}, \ldots, y_{k}\right) \\
& \tilde{\varrho}=\left(\tilde{\varrho}_{1}, \ldots, \tilde{\varrho}_{k}\right), \tilde{\eta}=\left(\tilde{\eta}_{1} \ldots, \tilde{\eta}_{k}\right), \tilde{\lambda}=\left(\tilde{\lambda}_{1}, \ldots, \tilde{\lambda}_{l}\right) .
\end{aligned}
$$

Theorem 2.10 Let $A \in L_{\mathrm{deg}}^{\mu}\left(M ; \mathbb{R}^{l}\right), B \in L_{\mathrm{deg}}^{\nu}\left(M ; \mathbb{R}^{l}\right)$, and let $A$ or $B$ be properly supported. Then we have $A B \in L_{\mathrm{deg}}^{\mu+\nu}\left(M ; \mathbb{R}^{l}\right)$ and $\sigma_{\psi}(A B)=$ $\sigma_{\psi}(A) \sigma_{\psi}(B)$.

Definition 2.11 An operator $A \in L_{\mathrm{deg}}^{\mu}\left(M ; \mathbb{R}^{l}\right)$ is said to be parameterdependent $\sigma_{\psi}$-elliptic, if $\sigma_{\psi}(A) \neq 0$ on $T^{*} Y^{0} \times \mathbb{R}^{l} \backslash 0$, moreover, if $\left.A\right|_{M \backslash Y^{k}} \in$ $L_{\mathrm{deg}}^{\mu}\left(M \backslash Y^{k} ; \mathbb{R}^{l}\right)$ is parameter-dependent $\sigma_{\psi^{-}}$elliptic on $M \backslash Y^{k} \in \mathfrak{M}_{k-1}$ (this notion is known by (IA)), and if in the above-mentioned splitting of variables near $Y^{k}$ the function $\tilde{\sigma}_{\psi}(A)$ is non-vanishing in $(\tilde{\varrho}, \xi, \tilde{\eta}, \tilde{\lambda}) \neq 0$ up to $r=(0, \ldots, 0)$. (For $l=0$ we simply speak about $\sigma_{\psi}$-ellipticity. $)$

Theorem 2.12 Let $M \in \mathfrak{M}_{k}$ and let $A \in L_{\mathrm{deg}}^{\mu}\left(M ; \mathbb{R}^{l}\right)$ be (parameterdependent) $\sigma_{\psi}$-elliptic. Then there is a properly supported parametrix $P \in$ $L_{\operatorname{deg}}^{-\mu}\left(M ; \mathbb{R}^{l}\right)$ in the sense that $1-P A, 1-A P \in L^{-\infty}\left(Y^{0} ; \mathbb{R}^{l}\right)$. 


\subsection{Symbols with twisted homogeneity}

If $M \in \mathfrak{M}_{k}, k \geq 1$, has a minimal statum $Y$ of dimension $q>0$, then the operators in the local model $X^{\Delta} \times \Omega$ of $M$ close to $Y, \Omega \subseteq \mathbb{R}^{q}$ open, $X \in \mathfrak{M}_{k-1}$, will be pseudo-differential operators on $\Omega$ with operator-valued amplitude functions, operating in distribution spaces over (the main stratum of) $X^{\Delta}$ and also over lower-dimensional strata. An important aspect in this connection is the twisted homogeneity (see Remark 1.5).

Definition 2.13 Let $H$ and $\widetilde{H}$ be Hilbert spaces, equipped with group actions $\kappa=\left\{\kappa_{\lambda}\right\}_{\lambda \in \mathbb{R}_{+}}$and $\tilde{\kappa}=\left\{\tilde{\kappa}_{\lambda}\right\}_{\lambda \in \mathbb{R}_{+}}$, respectively. Then $S^{(\mu)}\left(\Omega \times\left(\mathbb{R}^{q} \backslash\right.\right.$ $\{0\}) ; H, \widetilde{H}), \mu \in \mathbb{R}$, is defined to be the subspace of all $a(y, \eta) \in C^{\infty}(\Omega \times$ $\left.\left(\mathbb{R}^{q} \backslash\{0\}\right), \mathcal{L}(H, \widetilde{H})\right)$ such that

$$
a_{(\mu)}(y, \lambda \eta)=\lambda^{\mu} \tilde{\kappa}_{\lambda} a_{(\mu)}(y, \eta) \kappa_{\lambda}^{-1}
$$

for all $\lambda \in \mathbb{R}_{+}$. Here $\mathcal{L}(H, \widetilde{H})$ is the space of linear continuous operators $H \rightarrow \widetilde{H}$ in the operator norm topology.

Let $\chi(\eta)$ be an excision function in $\mathbb{R}_{\eta}^{q}$. Then, if $a_{(\mu)}(y, \eta)$ is as in Definition 2.13 , the function $a(y, \eta):=\chi(\eta) a_{(\mu)}(y, \eta)$ which belongs to

$$
C^{\infty}\left(\Omega \times \mathbb{R}^{q}, \mathcal{L}(H, \widetilde{H})\right)
$$

has the property that

$$
\sup _{\substack{y \in K \\ \eta \in \mathbb{R}^{q}}}\langle\eta\rangle^{-\mu+\beta \mid}\left\|\tilde{\kappa}_{\langle\tilde{\eta}\rangle}^{-1}\left\{D_{y}^{\alpha} D_{\eta}^{\beta} a(y, \eta)\right\} \kappa_{\langle\eta\rangle}\right\|_{\mathcal{L}(H, \widetilde{H})}
$$

is finite for every $\alpha, \beta \in \mathbb{N}^{q}$ and $K \Subset \Omega$. Let

$$
S^{\mu}\left(\Omega \times \mathbb{R}^{q} ; H, \widetilde{H}\right)
$$

denote the set of all $a(y, \eta)$ in the space (46) with that property. Moreover, let

$$
S_{\mathrm{cl}}^{\mu}\left(\Omega \times \mathbb{R}^{q} ; H, \widetilde{H}\right)
$$

denote the subspace of those $a(y, \eta)$ in the space (46) such that there are elements $a_{(\mu-j)}(y, \eta) \in S^{(\mu-j)}\left(\Omega \times\left(\mathbb{R}^{q} \backslash\{0\}\right) ; H, \widetilde{H}\right), j \in \mathbb{N}$, with

$$
a(y, \eta)-\sum_{j=0}^{N} \chi(\eta) a_{(\mu-j)}(y, \eta) \in S^{\mu-(N+1)}\left(\Omega \times \mathbb{R}^{q} ; H, \widetilde{H}\right)
$$


for all $N \in \mathbb{N}$. The functions in (48) are called (operator-valued) symbols, those in (49) classical symbols, and the $a_{(\mu-j)}(y, \eta), j \in \mathbb{N}$, the homogeneous components of $a(y, \eta)$. If a consideration refers to classical or general symbols, we also write as subscript ' $(\mathrm{cl})$ '. In particular, $S_{(\mathrm{cl})}^{\mu}\left(\mathbb{R}^{q} ; H, \widetilde{H}\right)$ denotes the space of symbols that are independent of $y$.

Example 2.14 (i) The operator $\mathcal{M}_{\varphi}$ of multiplication by $\varphi(r) \in C_{0}^{\infty}\left(\overline{\mathbb{R}}_{+}\right)$, represents an element in $S^{0}\left(\mathbb{R}^{q} ; K^{s, \gamma}\left(X^{\Delta}\right), K^{s, \gamma}\left(X^{\Delta}\right)\right)$ for every $s, \gamma \in$ $\mathbb{R}$.

(ii) If $A=r^{-\mu} \sum_{j+|\alpha| \leq \mu} a_{j \alpha}(r, y)\left(-r \partial_{r}\right)^{j}\left(r D_{y}\right)^{\alpha}$ is an edge-degenerate operator on $X^{\Delta} \times \Omega$ where the coefficients $a_{j \alpha} \in C^{\infty}\left(\overline{\mathbb{R}}_{+} \times \Omega\right.$, Diff $\left.{ }^{\mu-(j+|\alpha|)}(X)\right)$ are independent of $r$ for $r \geq C$ for some $C>0$, then we have

$$
a(y, \eta) \in S^{\mu}\left(\Omega \times \mathbb{R}^{q} ; K^{s, \gamma}\left(X^{\Delta}\right), K^{s-\mu, \gamma-\mu}\left(X^{\Delta}\right)\right)
$$

for every $s, \gamma \in \mathbb{R}$. If all $a_{j \alpha}$ are independent of $r$, the symbol $a(y, \eta)$ is classical. In that case we have $a_{(\mu)}(y, \eta)=\sigma_{\wedge}(A)(y, \eta)$.

Forming $\operatorname{Op}(a) u(y)=\iint e^{i\left(y-y^{\prime}\right) \eta} a(y, \eta) u\left(y^{\prime}\right) d y^{\prime} d \eta, d \eta=(2 \pi)^{-q} d \eta$, for every $a(y, \eta)$ in (48) we obtain a continuous operator

$$
\mathrm{Op}(a): C_{0}^{\infty}(\Omega, H) \rightarrow C^{\infty}(\Omega, \widetilde{H})
$$

Instead of $\mathrm{Op}(\cdot)$ we also write $\mathrm{Op}_{y}(\cdot)$.

Theorem 2.15 Let $a(y, \eta) \in S^{\mu}\left(\Omega \times \mathbb{R}^{q}, H, \widetilde{H}\right)$; then (50) extends to continuous operators

$$
\mathrm{Op}(a): \mathcal{W}_{\text {comp }}^{s}(\Omega, H) \rightarrow \mathcal{W}_{\text {loc }}^{s-\mu}(\Omega, \widetilde{H})
$$

for all $s \in \mathbb{R}$.

This theorem has been proved (under very general assumptions on the symbols) in [89], generalising the approach of [30] in the scalar case. For the concrete contexts of the edge calculus it was known before; a proof under some mild additional assumptions may be found, e.g., in [72], see also [14].

\subsection{Conical exits to infinity}

As we see in the example of edge symbols (see Proposition 1.18 or the formula (38)), edge-degenerate operators give rise to operators on an infinite cone $X^{\Delta}$, where (apart from the interesting behaviour near the tip) we should refer to some background information at infinity. Although freezing of coefficients at 
the edge, say in the expression for $\sigma_{\wedge}(A)(y, \eta), \eta \neq 0$, shows an automatic behaviour at infinity which is completely determined by the behaviour of the operator close to the edge, it is worth to notice a connection to aspects of the (pseudo-differential) calculus on a manifold with conical exit to infinity. This is remarkable even when $X$ smooth; however, if $X$ itself has singularities, the various strata of $X^{\Delta}$ (except for the tip of the cone) also have conical exits to infinity, i.e., the geometric singularities are 'travelling' to infinity and do something specific in respect to the details of the calculus.

To illustrate things we consider the Euclidean space $\mathbb{R}^{m} \ni x$ interpreted as a cone $\left(S^{m-1}\right)^{\Delta}$ with conical exit $|x| \rightarrow \infty$. The symbols of the calculus in this case are required to satisfy the estimates

$$
\left|D_{x}^{\alpha} D_{\xi}^{\beta} a(x, \xi)\right| \leq c\langle x\rangle^{\nu-|\alpha|}\langle\xi\rangle^{\mu-\beta}
$$

for all $x, \xi \in \mathbb{R}^{m}, \alpha, \beta \in \mathbb{N}^{m}, c=c(\alpha, \beta)>0$.

Given a pair of orders $(\mu, \nu) \in \mathbb{R}^{2}$ we obtain in this way a space of symbols $S^{\mu ; \nu}\left(\mathbb{R}^{m} \times \mathbb{R}^{m}\right)$, see [91], [62], [9]. The associated pseudo-differential operators in $\mathbb{R}^{m}$ act in weighted Sobolev spaces $H^{s ; g}\left(\mathbb{R}^{m}\right):=\langle x\rangle^{-g} H^{s}\left(\mathbb{R}^{m}\right)$ as continuous operators

$$
\mathrm{Op}_{x}(a): H^{s ; g}\left(\mathbb{R}^{m}\right) \rightarrow H^{s-\mu ; g-\nu}\left(\mathbb{R}^{m}\right)
$$

for every $s, g \in \mathbb{R}$. In particular, we obtain a continuous operator $\mathcal{S}\left(\mathbb{R}^{m}\right) \rightarrow$ $\mathcal{S}\left(\mathbb{R}^{m}\right)$ in the Schwartz space. By virtue of $\langle x\rangle^{-\nu} S^{\mu ; \nu}\left(\mathbb{R}^{m} \times \mathbb{R}^{m}\right)=S^{\mu ; 0}\left(\mathbb{R}^{m} \times\right.$ $\mathbb{R}^{m}$ ) the order $\nu$ at $x=\infty$ is not very essential in this context. Therefore, we often may assume $\nu=0$.

It can be proved that $\mathrm{Op}(\cdot)$ defines a bijection

$$
\text { Op }: S^{\mu ; \nu}\left(\mathbb{R}^{m} \times \mathbb{R}^{m}\right) \rightarrow\left\{\mathrm{Op}(a): a(x, \xi) \in S^{\mu ; \nu}\left(\mathbb{R}^{m} \times \mathbb{R}^{m}\right)\right\}=: L^{\mu ; \nu}\left(\mathbb{R}^{m}\right)
$$

to the space of associated pseudo-differential operators, and that

$$
L^{-\infty ;-\infty}\left(\mathbb{R}^{m}\right):=\bigcap_{\mu, \nu \in \mathbb{R}} L^{\mu ; \nu}\left(\mathbb{R}^{m}\right)
$$

coincides with the space of all integral operators with kernels in $\mathcal{S}\left(\mathbb{R}^{m} \times \mathbb{R}^{m}\right)$.

An operator $A=\operatorname{Op}(a) \in L^{\mu ; \nu}\left(\mathbb{R}^{m}\right)$ is said to be elliptic, if there exists a $p(x, \xi) \in S^{-\mu ;-\nu}\left(\mathbb{R}^{m} \times \mathbb{R}^{m}\right)$ such that

$$
1-p(x, \xi) a(x, \xi) \in S^{-1 ;-1}\left(\mathbb{R}^{m} \times \mathbb{R}^{m}\right) .
$$

Theorem 2.16 The following conditions are equivalent:

(i) A ist elliptic; 
(ii) the operator (51) is Fredholm for some fixed $(s, g)=\left(s_{0}, g_{0}\right)$.

An elliptic operator $A \in L^{\mu ; \nu}\left(\mathbb{R}^{m}\right)$ has a parametrix $P \in L^{-\mu ;-\nu}\left(\mathbb{R}^{m}\right)$, and (51) is Fredholm for all $s, g \in \mathbb{R}$. Moreover, $V:=\operatorname{ker} A \subset \mathcal{S}\left(\mathbb{R}^{m}\right)$ is independent of $s, g$, and there is a subspace $W \subset \mathcal{S}\left(\mathbb{R}^{m}\right)$ of finite dimension such that $W \cap \operatorname{im} A=\{0\}, W+\operatorname{im} A=H^{s-\mu ; g-\nu}\left(\mathbb{R}^{m}\right)$, for all $s, g$.

Remark 2.17 Let $A \in L^{\mu ; \nu}\left(\mathbb{R}^{m}\right)$ induce an isomorphism (51) for some $(s, g)=\left(s_{0}, g_{0}\right)$, then (51) is an isomorphism for all $s, g \in \mathbb{R}$, and we have $A^{-1} \in L^{-\mu ;-\nu}\left(\mathbb{R}^{m}\right)$.

There is also an analogue of classical symbols, including the variable $x$. Using $S_{\mathrm{cl}}^{\mu}\left(\mathbb{R}^{m}\right)$ with its nuclear Fréchet topology, we can set

$$
S_{\mathrm{cl}_{\xi ; x}}^{\mu ; \nu}\left(\mathbb{R}^{m} \times \mathbb{R}^{m}\right):=S_{\mathrm{cl}_{\xi}}^{\mu}\left(\mathbb{R}^{m}\right) \hat{\otimes}_{\pi} S_{\mathrm{cl}_{x}}^{\nu}\left(\mathbb{R}_{x}^{m}\right)
$$

( $\hat{\otimes}_{\pi}$ denotes the projective tensor product of Fréchet spaces).

There is a straightforward generalisation of these considerations to the case of a $C^{\infty}$ manifold $M$ with conical exit to infinity. That means, $M$ can be written as a union

$$
M=M_{0} \cup M_{\infty}
$$

where $M_{0}$ is $C^{\infty}$ and $M_{\infty}$ is identified with an infinite cylinder $(\varepsilon, \infty) \times X$ for some $0<\varepsilon<1$ and a (for simplicity) closed compact $C^{\infty}$ manifold $X$, such that $M_{\infty} \cap M_{0}=(\varepsilon, \delta) \times X$ for a $\delta$ with $0<\varepsilon<\delta<1$ (i.e., $M_{0}$ is a kind of bottle with a neck, containing the cylinder $(\varepsilon, \delta) \times X$, and $M_{\infty}$ is connected with $M_{0}$ along this cylinder). In addition on $M_{\infty}$ we fix an atlas of charts $\chi:(\varepsilon, \infty) \times U \rightarrow \Gamma$ for coordinate neighbourhoods $U$ on $X$ and a set $\Gamma \subset \mathbb{R}^{n+1}$ (for $n=\operatorname{dim} X$ ) of the form $\Gamma=\left\{\left(x_{0}, x\right) \in \mathbb{R}^{n+1}: x_{0} \in(\varepsilon, \infty)\right.$, $x / x_{0} \in B$ for an open bounded set $\left.B \subset \mathbb{R}^{n}\right\}$, such that $\chi(\lambda r, \cdot)=\lambda \chi(r, \cdot)$ for all $\lambda \geq 1,(r, \cdot) \in(\varepsilon, \infty) \times U$. There is then a variant of the above-mentioned symbol spaces for $\Gamma$ instead of $\mathbb{R}^{m}$, namely, $S^{\mu ; \nu}\left(\Gamma \times \mathbb{R}^{m}\right)$, and there are also subspaces of classical (in $(x, \xi))$ symbols indicated by subscript ' $\mathrm{cl}_{\xi ; x}$ '. Using an open covering of $M$ by charts, over $M_{\infty}$ as just explained, and over $M_{0}$ arbitrary, we can define the space $L_{(\mathrm{cl})}^{\mu ; \nu}(M)$ of pseudo-differential operators as sums of local pseudo-differential operators with such symbols, pulled back to $M$ and combined with localising factors, plus global smoothing operators. The latter ones are defined by the mapping properties $H^{s ; g}(M) \rightarrow H^{s^{\prime} ; g^{\prime}}(M)$ for all $s, s^{\prime}, g, g^{\prime} \in \mathbb{R}$; the weighted Sobolev spaces $H^{s ; g}(M)$ are an immediate generalisation of the ones in $\mathbb{R}^{m}$ (assuming that $M \backslash M_{\infty}$ is compact, otherwise we have suitable 'comp' and 'loc' versions). 
Definition 2.18 Let $M$ be a manifold with conical exit to infinity. An $A \in$ $L^{\mu ; \nu}(M)$ is said to be elliptic, if $A$ is elliptic as an operator in $L^{\mu}(M)$, and if for the local symbols $a(x, \xi) \in S^{\mu ; \nu}\left(\Gamma \times \mathbb{R}^{m}\right)$ there are $p(x, \xi) \in S^{-\mu ;-\nu}\left(\Gamma \times \mathbb{R}^{m}\right)$ such that $1-p(x, \xi) a(x, \xi) \in S^{-1 ;-1}\left(\Gamma \times \mathbb{R}^{m}\right)$ (the second condition concerns the above-mentioned charts $\chi:(\varepsilon, \infty) \times U \rightarrow \Gamma$ and will be referred to a 'exit-ellipticity').

Remark 2.19 There is a straightforward generalisation of Theorem 2.16 to the case of an arbitrary manifold $M$ with conical exit to infinity (when $M \backslash M_{\infty}$ is compact). Also the cylinder $M_{\infty}=(\varepsilon, \infty) \times X \ni(r, x)$ itself is a manifold with conical exit to infinity, though open for $r \rightarrow \varepsilon$, but it makes sense to consider operators of the class $L_{(\mathrm{cl})}^{\mu ; \nu}\left(M_{\infty}\right)$ also in this case. Ellipticity then entails the existence of a parametrix in $L_{(\mathrm{cl})}^{-\mu ;-\nu}\left(M_{\infty}\right)$.

Remark 2.20 Let $X$ be closed compact, and consider the set $X^{\Delta} \backslash\{([0, \varepsilon] \times$ $X) /(\{0\} \times X)\}=: X_{\asymp, \varepsilon}$ as a part of a manifold with conical exit to infinity, for instance, $\mathbb{R} \times X=: X_{\asymp}$ which has another exit $r \rightarrow-\infty$. Then we have

$$
(1-\omega) H^{s ; g}\left(X_{\asymp}\right)=(1-\omega)\langle r\rangle^{-g} \mathcal{K}^{s, \gamma}\left(X^{\Delta}\right)
$$

for any cut-off function $\omega$ (where $1-\omega(r):=0$ for $r<0$ ), and, in particular, $H_{\text {cone }}^{s}\left(X^{\wedge}\right)=\left.H^{s ; 0}\left(X_{\asymp}\right)\right|_{\mathbb{R}_{+} \times X}$.

Theorem 2.21 Let $M$ be a manifold with edge (see Definition 1.14), and let $A \in \operatorname{Diff}_{\mathrm{deg}}^{\mu}(M)$ be $\sigma_{\psi}$-elliptic (see Definition 1.21). Then the operator $\sigma_{\wedge}(A)(y, \eta)$ for every fixed $y$ and $\eta \neq 0$ is elliptic in $L^{\mu ; 0}\left(X_{\asymp}, \varepsilon\right)$ for every $\varepsilon>0$ (see the notation of Remark 2.20). 


\section{A hierarchy of operator algebras}

\subsection{The program of the iterative calculus}

Given a space $N \in \mathfrak{M}_{k-1}, k \in \mathbb{N}, k \geq 2$, we assume to have constructed an algebra $\mathfrak{A}(N)$ of operators on the maximal stratum of $N$, graded by orders $\mu \in \mathbb{R}$, i.e., $\mathfrak{A}(N)=\bigcup_{\mu} \mathfrak{A}^{\mu}(N)$, where every $\mathfrak{A}^{\mu}(N)$ is again the union of subspaces $\mathfrak{A}^{\mu}(N, \boldsymbol{g})$ with weight data $\boldsymbol{g}=(\gamma, \gamma-\mu)$ for a tuple of weights $\gamma=\left(\gamma_{1}, \ldots, \gamma_{k-1}\right) \in \mathbb{R}^{k-1}, \gamma-\mu=\left(\gamma_{1}-\mu, \ldots, \gamma_{k-1}-\mu\right)$. For $N \in \mathfrak{M}_{0}$ we have in mind the spaces $\mathfrak{A}^{\mu}(N)=L_{\text {cl }}^{\mu}(N)$ without any such weight data, while for $N \in \mathfrak{M}_{1}$ we mean the respective cone or edge pseudodifferential algebras, established in [71], [72], etc. (see also the references in the introduction). In general we construct $\mathfrak{A}(N)$ as a subspace of $L_{\mathrm{deg}}^{\mu}(N)$ in such a way that $\mathfrak{A}^{\mu}(N)=L_{\text {deg }}^{\mu}(N) \bmod L^{-\infty}$ on the maximal stratum of $N$ (see Definition 2.8). For a more transparent description, we consider at the moment operators of the form of upper left corners rather than full $2 \times 2$ block matrices including trace and potential entries occurring in the edge case, (for some reason this is not too restrictive, see Remark 3.8 below). In addition we consider operators between spaces of scalar distributions, although in general it is interesting to admit distributional sections in vector bundles (which is a straightforward generalisation).

The program of the iterative calculus on spaces $M \in \mathfrak{M}_{l}$ for $l \geq k$ is to organise a natural scenario to pass from $\mathfrak{A}(N)$ to corresponding higher generations of calculi. Spaces $M$ in $\mathfrak{M}_{k}$ can be obtained from $\mathfrak{M}_{k-1}$ by pasting together local cones $N^{\Delta}$ or wedges $N^{\Delta} \times \Omega, \Omega \subseteq \mathbb{R}^{q}$ open, $N \in \mathfrak{M}_{k-1}$. Analytically, the main steps (apart from invariance aspects) consist in understanding the correspondence between $\mathfrak{A}(N)$ and the next higher algebras

$$
\mathfrak{A}\left(N^{\Delta}\right) \text { and } \mathfrak{A}\left(N^{\Delta} \times \Omega\right) .
$$

We will call the process of passing from $\mathfrak{A}(N)$ to (52) 'conification' and 'edgification', respectively, of $\mathfrak{A}(N)$. The key words listed below characterise the main elements of this approach. The corresponding structures are feeded in as information by (IA) (induction assumption). Those are to be generated again on the next floor of the building of operator algebras.

Parameter-dependent calculus. The first observation is that (by (IA)) we have a parameter-dependent version $\mathfrak{A}\left(N ; \mathbb{R}^{l}\right)=\bigcup \mathfrak{A}^{\mu}\left(N, \boldsymbol{g} ; \mathbb{R}^{l}\right)$ of $\mathfrak{A}(N)$ with parameters $\lambda=\left(\lambda_{1}, \ldots, \lambda_{l}\right) \in \mathbb{R}^{l}$ of dimension $l \in \mathbb{N}$. The parameters can be introduced by letting local symbols depend on those additional parameters, treated as components of covariables, and by defining parameter-dependent smoothing operators as Schwartz functions in the sense $\mathcal{S}\left(\mathbb{R}^{l}, \mathfrak{A}^{-\infty}(N)\right.$ ) (see also the constructions of Section 2.2). Here $\mathfrak{A}^{-\infty}(N)$ 
denotes the space of smoothing operators on $N$, consisting of the spaces $\mathfrak{A}^{-\infty}(N, \boldsymbol{g}) \ni C$, required to map weighted distributions of any smoothness to weighted smooth functions with (here continuous) asymptotics; the same is asked on the formal adjoint $C^{*}$ (see the explanations below under the headline 'Global smoothing operators'. Then $\mathfrak{A}^{-\infty}(N, \boldsymbol{g})$ has a natural locally convex topology, and we can talk about Schwartz functions with values there. As a parameter we also may have $\operatorname{Im} w$ on any line $\Gamma_{\beta}:=\{w \in \mathbb{C}: \operatorname{Re} w=\beta\}$; then our notation is, e.g., $\mathfrak{A}^{\mu}\left(N, \boldsymbol{g} ; \Gamma_{\beta} \times \mathbb{R}^{l}\right)$.

Cones with exit to infinity. If $\mathfrak{A}(N)$ is established for every $N \in$ $\mathfrak{M}_{k-1}$, we know at the same time $\mathfrak{A}\left(N^{\wedge}\right)$ for $N^{\wedge}=\mathbb{R}_{+} \times N \ni(t, \cdot)$, since $\mathfrak{M}_{k-1}$ is closed under taking Cartesian products with a $C^{\infty}$ manifold. However, the behaviour for $t \rightarrow \infty$ which is regarded as a conical exit of $N^{\wedge}$ to infinity, can be specified in terms of extra conditions on symbols as well as on smoothing operators. This gives rise to what we call exit symbols, where, roughly speaking, $t$ is recognised as a covariable. In particular, we may have components of homogeneity zero in $t$ at $t \rightarrow \infty$, with values in spaces of symbols in the covariables in the 'usual sense'. The smoothing operators are defined in terms of kernels that are Schwartz functions in $\left(t, t^{\prime}\right)$ for large $t, t^{\prime}$ with values in $\mathfrak{A}^{-\infty}(N)$. Let $\mathfrak{A}_{\text {cone }}\left(N^{\wedge}\right)$ denote this subcalculus of $\mathfrak{A}\left(N^{\wedge}\right)$.

Holomorphic Mellin symbols and kernel cut-off. As explained before, together with the operator spaces $\mathfrak{A}(N)$ we have parameter-dependent analogues $\mathfrak{A}\left(N ; \mathbb{R}^{l}\right)$ and also families in $C^{\infty}\left(\overline{\mathbb{R}}_{+} \times \Omega, \mathfrak{A}\left(N ; \mathbb{R}^{l}\right)\right)$ for any open set $\Omega \subseteq \mathbb{R}^{q}$. This allows us to define edge-degenerate families

$$
p(t, z, \tau, \zeta):=\tilde{p}(t, z, t \tau, t \zeta)
$$

for $\tilde{p}(t, z, \tilde{\tau}, \tilde{\zeta}) \in C^{\infty}\left(\overline{\mathbb{R}}_{+} \times \Omega, \mathfrak{A}\left(N ; \mathbb{R}_{\tilde{\tau}, \tilde{\zeta}}^{1+q}\right)\right)$. The combination $t \tau$ indicates an operation of Fuchs type in $t \in \mathbb{R}_{+}$, while $t \zeta$ represents edge-degenerate derivatives. For the calculus with Mellin symbols, analogously as in the edge algebra for $k=1$, we should impose quantisations based on a holomorphic dependence on the Mellin covariable in the new axial direction $t \in \mathbb{R}_{+}$. So the point is to extend the notion of parameter-dependent operator spaces $\mathfrak{A}\left(N ; \mathbb{R}^{l+1}\right)$ to the case $\mathfrak{A}\left(N ; \mathbb{C} \times \mathbb{R}^{l}\right), l \in \mathbb{N}$. The property is that any $h(w, \lambda) \in \mathfrak{A}\left(N ; \mathbb{C} \times \mathbb{R}^{l}\right)$ is defined by the condition $h(w, \lambda) \in \mathcal{A}\left(\mathbb{C}, \mathfrak{A}\left(N ; \mathbb{R}^{l}\right)\right)$ (with $\mathcal{A}(U, E)$ for open $U \subseteq \mathbb{C}$ denoting the space of holomorphic functions in $U$ with values in $E$ ), such that

$$
h(\beta+i \tau, \lambda) \in \mathfrak{A}\left(N ; \mathbb{R}_{\tau, \lambda}^{l+1}\right)
$$

for every $\beta \in \mathbb{R}$, uniformly in arbitrary compact $\beta$-intervals. The holomorphy of operator families can be defined in terms of holomorphic families of 
the underlying local symbols, plus holomorphy of families of smoothing operators, formulated by using the mapping properties between weighted spaces and spaces of smooth functions (say, with continuous asymptotics).

A kernel cut-off construction (see the terminology in [70] or [72]) tells us that for every $f(w, \lambda) \in \mathfrak{A}\left(N ; \Gamma_{\beta} \times \mathbb{R}^{l}\right), \beta \in \mathbb{R}$ fixed, there exists an $h(w, \lambda) \in \mathfrak{A}\left(N ; \mathbb{C} \times \mathbb{R}^{l}\right)$ such that

$$
\left.h(w, \lambda)\right|_{\Gamma_{\beta} \times \mathbb{R}^{l}}=f(w, \lambda) \bmod \mathfrak{A}^{-\infty}\left(N ; \Gamma_{\beta} \times \mathbb{R}^{l}\right) .
$$

It holds for the singularity order $k=1$; then it follows for $k=2$. By (IA) it is imposed up to the singularity order $k-1$.

If we want to point out subspaces of $\mathfrak{A}\left(N ; \mathbb{C} \times \mathbb{R}^{q}\right)$ of operators of order $\mu$ and involved weight data $(\gamma, \gamma-\mu)$, we write

$$
\mathfrak{A}^{\mu}\left(N,(\gamma, \gamma-\mu) ; \mathbb{C} \times \mathbb{R}^{q}\right) .
$$

Similar notation is used for other variants of our spaces.

Mellin quantisation. The Mellin quantisation consists of the theorem that for every given

$$
\tilde{p}(t, z, \tilde{\tau}, \tilde{\zeta}) \in C^{\infty}\left(\overline{\mathbb{R}}_{+} \times \Omega, \mathfrak{A}\left(N ; \mathbb{R}_{\tilde{\tau}, \tilde{\zeta}}^{1+q}\right)\right)
$$

there exists an

$$
\tilde{h}(t, z, w, \tilde{\zeta}) \in C^{\infty}\left(\overline{\mathbb{R}}_{+} \times \Omega, \mathfrak{A}\left(N ; \mathbb{C}_{w} \times \mathbb{R}_{\tilde{\zeta}}^{q}\right)\right)
$$

such that, when we set

$$
p(t, z, \tau, \zeta):=\tilde{p}(t, z, t \tau, t \zeta), h(t, z, w, \zeta):=\tilde{h}(t, z, w, t \zeta)
$$

we have

$$
\mathrm{op}_{M}^{\gamma}(h)(z, \zeta)=\mathrm{Op}_{t}(p)(z, \zeta)
$$

$\bmod C^{\infty}\left(\Omega, \mathfrak{A}^{-\infty}\left(N^{\wedge} ; \mathbb{R}^{q}\right)\right)$. Here $\operatorname{op}_{M}^{\gamma}(\cdot)$ is the pseudo-differential operator on $\mathbb{R}_{+}$, based on the weighted Mellin transform $M_{\gamma} u(w)=M\left(t^{-\gamma} u\right)(w+\gamma)$; then

$$
\mathrm{op}_{M}^{\gamma}(f):=M_{\gamma}^{-1} f M_{\gamma}=t^{\gamma} M^{-1}\left(T^{-\gamma} f\right) M t^{-\gamma},
$$

$\left(T^{-\gamma} f\right)(w):=f(w-\gamma), M:=M_{0}$. This result which is true of the singularity order $k=1$ is imposed as (IA) in the iterative process. Note that, although the 'Fourier phase function' $\left(t-t^{\prime}\right) \tau$ in $\mathrm{Op}_{t}(\cdot)$ is equivalent (in the sense of Fourier distributions) to the 'Mellin phase function' $\left(\log t^{\prime}-\log t\right) \tau$ which is involved in $\operatorname{op}_{M}^{\gamma}(\cdot)$, the correspondence $\tilde{p} \rightarrow \tilde{h}$,

$$
C^{\infty}\left(\overline{\mathbb{R}}_{+} \times \Omega, \mathfrak{A}\left(N ; \mathbb{R}^{1+q}\right)\right) \rightarrow C^{\infty}\left(\overline{\mathbb{R}}_{+} \times \Omega, \mathfrak{A}\left(N ; \mathbb{C} \times \mathbb{R}^{q}\right)\right)
$$


is more than only to find a new amplitude function for the new phase. In fact, we do not only save the smoothness in $t$ up to zero but obtain holomorphy in the axial covariable.

Edge quantisation By edge quantisation we understand a specific way of passing from an edge-degenerate family

$$
p(t, z, \tau, \zeta)=\tilde{p}(t, z, t \tau, t \zeta),
$$

(cf. (53)) to an operator in weighted edge spaces. Applying the Mellin quantisation we first find a Mellin amplitude function $h(t, z, w, \zeta)$ (see (54)). In addition we set

$$
p_{0}(t, z, \tau, \zeta):=\tilde{p}(0, z, t \tau, t \zeta), h_{0}(t, z, w, \zeta):=\tilde{h}(0, z, w, t \zeta) .
$$

Moreover, we fix cut-off functions $\omega, \tilde{\omega}, \tilde{\tilde{\omega}}$ on the half-axis such that $\tilde{\tilde{\omega}} \prec \omega \prec$ $\tilde{\omega}$ and cut-off functions $\sigma, \tilde{\sigma}(\omega \prec \tilde{\omega}$ means that $\tilde{\omega} \equiv 1$ on $\operatorname{supp} \omega)$. We set

$$
a_{M}(z, \zeta):=t^{-\mu} \omega(t[\zeta]) \operatorname{op}_{M}^{\delta-\frac{n}{2}}(h)(z, \zeta) \tilde{\omega}\left(t^{\prime}[\zeta]\right)
$$

for a $\delta \in \mathbb{R}, n:=\operatorname{dim} N$, and

$$
a_{\psi}(z, \zeta):=t^{-\mu}(1-\omega(t[\zeta])) \omega_{0}\left(t[\zeta], t^{\prime}[\zeta]\right) \operatorname{Op}_{t}(p)(z, \zeta)\left(1-\tilde{\tilde{\omega}}\left(t^{\prime}[\zeta]\right)\right) .
$$

Here $t^{\prime}$ is the variable in the argument functions $u\left(t^{\prime}, \cdot\right)$, while $\omega_{0}\left(t, t^{\prime}\right):=$ $\psi\left(\left(t-t^{\prime}\right)^{2} /\left(1+\left(t-t^{\prime}\right)^{2}\right)\right)$ for any $\psi \in C_{0}^{\infty}\left(\overline{\mathbb{R}}_{+}\right)$such that $\psi(t)=1$ for $t<\frac{1}{2}$, $\psi(t)=0$ for $t>\frac{2}{3}$. We now form the operator-valued amplitude function

$$
a(z, \zeta):=\sigma\left\{a_{M}(z, \zeta)+a_{\psi}(z, \zeta\} \tilde{\sigma}\right.
$$

(with $\tilde{\sigma}$ depending on $t^{\prime}$ ). The number $\delta$ plays the role of the new weight $\gamma_{k}$ for the $k$-th axial variable $t \in \mathbb{R}_{+}$. The operator $\mathrm{Op}_{z}(a)$ is just the result of edge quantising the (operator-valued) amplitude function (55) up to the factors $\sigma, \tilde{\sigma}$ which localise the operator near the edge). The quantisation rule (57) which produces $a_{\psi}$ can be slightly simplified for the case of singularity order $k=1$ by omitting $\omega_{0}$ in the middle (see [48]). However, for higher order singularities this localising factor seems to be indispensable, see [8] or [48]. Note that for $k=1$ (and $\operatorname{dim} Y>0$ ) there is also another edge quantisation of a simpler shape, see [20]. However, it seems that an analogue for $k>1$ (even if it is possible) causes more effort than the quantisation (58).

The asymptotic part of the corner calculus. The following constructions concern the case of compact $X \in \mathfrak{M}_{k-1}$. Considering the wedge $X^{\Delta} \times \Omega$, or its stretched version

$$
\mathbb{R}_{+} \times X \times \Omega \ni(t, \cdot, z),
$$


the above constructions contribute to building up the calculus of $\mathfrak{A}\left(X^{\Delta} \times \Omega\right)$. There is another essential ingredient, namely, so-called Green and 'smoothing' Mellin operators which are pseudo-differential operators on $\Omega$ (the edge) with operator-valued symbols

$$
g(z, \zeta) \text { and } m(z, \zeta)
$$

respectively. A Green symbol associated with the weight data $\boldsymbol{g}=((\gamma, \delta),(\gamma-$ $\mu, \delta-\mu))$ is defined by

$$
g(z, \zeta) \in S_{\mathrm{cl}}^{\mu}\left(\Omega \times \mathbb{R}^{q} ; K^{s,(\gamma, \delta)}\left(X^{\Delta}\right), \mathcal{S}_{P}^{(\gamma-\mu, \delta-\mu)}\left(X^{\Delta}\right)\right),
$$

together with a similar property of the pointwise formal adjoint, for all $s \in \mathbb{R}$ (the definition of symbols is based on a natural extension of the corresponding notion from Section 2.3 to the case of Fréchet spaces with group action, see also Remark 1.28). Here $\mathcal{S}_{P}^{(\gamma-\mu, \delta-\mu)}\left(X^{\Delta}\right)$ consists of Schwartz functions for $t \rightarrow \infty$ and weighted functions for $t \rightarrow 0$ with (say, continuous) asymptotics of type $P$ (see Section 1.1 and Section 3.3 below). Moreover, a smoothing Mellin symbol is a linear combination of expressions

$$
m(z, \zeta):=t^{-\mu+j} \omega(t[\zeta]) \operatorname{op}_{M}^{\delta_{j}-\frac{n}{2}}\left(f_{j \alpha}\right)(z) \zeta^{\alpha} \tilde{\omega}\left(t^{\prime}[\zeta]\right)
$$

$j \in \mathbb{N}, \alpha \in \mathbb{N}^{q},|\alpha| \leq j$, with arbitrary cut-off functions $\omega, \tilde{\omega}$, weights $\delta_{j} \in \mathbb{R}$, such that $\delta>\delta_{j}>\delta-j$, and Mellin symbols $f_{j \alpha} \in C^{\infty}\left(\Omega, \mathfrak{A}^{-\infty}\left(X ; \mathbb{C} \backslash V_{j}\right)\right)$. Here $V_{j} \subset \mathbb{C}$ is a closed subset (a so-called 'continuous' asymptotic type of Mellin symbols) such that $w_{0}, w_{1} \in V_{j}$ with Re $w_{0}=\operatorname{Re} w_{1}$ implies $(1-\alpha) w_{0}+$ $\alpha w_{1} \in V_{j}$ for every $0 \leq \alpha \leq 1,\left\{c_{0} \leq \operatorname{Re} w \leq c_{1}\right\} \cap V_{j}$ compact for every $c_{0} \leq c_{1}$, and $\Gamma_{\frac{n+1}{2}-\delta_{j}} \cap V_{j}=\emptyset$. The space $\mathfrak{A}^{-\infty}(X ; \mathbb{C} \backslash V)$ for such a $V$ is defined to be the set of all $f(w) \in \mathfrak{A}^{-\infty}\left(X ; \Gamma_{\frac{n+1}{2}-\delta}\right)$ which extend to a holomorphic $\mathfrak{A}^{-\infty}(X)$-valued function in $\mathbb{C} \backslash V$ such that $\left.\chi_{V} f\right|_{\Gamma_{\beta}} \in \mathfrak{A}^{-\infty}\left(X ; \Gamma_{\beta}\right)$ for every $\beta \in \mathbb{R}$ and every $\chi_{V} \in C^{\infty}(\mathbb{C})$ such that $\chi_{V}(w)=0$ for $\operatorname{dist}(w, V)<\varepsilon_{0}$, $\chi_{V}(w)=1$ for $\operatorname{dist}(w, V)>\varepsilon_{1}$ for some $0<\varepsilon_{0}<\varepsilon_{1}$. Recall that, by notation, $\mathfrak{A}^{-\infty}(N ; \mathbb{C} \backslash V)=\bigcup \mathfrak{A}^{-\infty}(X,(\gamma, \gamma-\mu), \boldsymbol{g} ; \mathbb{C} \backslash V)$ with the union over $(\gamma, \gamma-\mu)$ for $\gamma \in \mathbb{R}^{k-1}, \mu \in \mathbb{R}$. Operator functions $m(z, \zeta)$ represent symbols

$$
m(z, \zeta) \in S_{\mathrm{cl}}^{\mu}\left(\Omega \times \mathbb{R}^{q} ; K^{s,(\gamma, \delta)}\left(X^{\Delta}\right), K^{\infty,(\gamma-\mu, \delta-\mu)}\left(X^{\Delta}\right)\right)
$$

and

$$
m(z, \zeta) \in S_{\mathrm{cl}}^{\mu}\left(\Omega \times \mathbb{R}^{q} ; K_{P}^{s,(\gamma, \delta)}\left(X^{\Delta}\right), \mathcal{S}_{Q}^{(\gamma-\mu, \delta-\mu)}\left(X^{\Delta}\right)\right)
$$


for every (continuous) asymptotic type $P$ with some resulting $Q$. The pointwise formal adjoints have a similar property. (Concerning the spaces $K^{s,(\gamma, \delta)}\left(X^{\Delta}\right)$ and subspaces with asymptotics, see Section 1.1 and Section 3.3 below.) The operators $\operatorname{Op}_{z}(m+g)$ then constitute the local contribution near the edge of the asymptotic part of the operator space $\mathfrak{A}^{\mu}(M, \boldsymbol{g})$ for $M \in \mathfrak{M}_{k}$, $\boldsymbol{g}=((\gamma, \delta),(\gamma-\mu, \delta-\mu))$, locally modelled on $X^{\Delta} \times \Omega$.

Global smoothing operators. Given $M \in \mathfrak{M}_{k}, \delta, \mu \in \mathbb{R}, \gamma \in \mathbb{R}^{k-1}$, the space $\mathfrak{A}^{-\infty}(M,((\gamma, \delta),(\gamma-\mu, \delta-\mu))$ is defined to be the set of all

$$
C: H_{[\mathrm{comp})}^{s,(\gamma, \delta)}(M) \rightarrow H_{[\mathrm{loc}), P}^{s^{\prime},(\gamma-\mu, \delta-\mu)}(M)
$$

that are continuous for arbitrary $s, s^{\prime} \in \mathbb{R}$, with some asymptotic type $P$, and $C^{*}$, the formal adjoint, has a similar property. In the case $\operatorname{dim} Y=0$ (i.e., when $M$ is locally near $Y=\{v\}$ modelled on a cone $X^{\Delta}$ for an $X \in \mathfrak{M}_{k-1}$, such a $C$ is also called a Green operator of the corresponding higher cone calculus.

Global corner operators of $k$-th generation. An operator $A \in$ $\mathfrak{A}^{\mu}(M, \boldsymbol{g})$ for $\boldsymbol{g}=\left(((\gamma, \delta),(\gamma-\mu, \delta-\mu)), M \in \mathfrak{M}_{k}\right.$, is defined as follows: We first choose cut-off functions $\sigma, \tilde{\sigma}$ on $M$ that are equal to 1 in a neighbourhood of the minimal stratum $Y \subset M$ and vanish outside another neighbourhood of $Y$. By virtue of $N:=M \backslash Y \in \mathfrak{M}_{k-1}$ we have the spaces $\mathfrak{A}^{\mu}(N ;(\gamma, \gamma-\mu))$, known by (IA). Then $\mathfrak{A}^{\mu}(M, \boldsymbol{g})$ is defined to be the set of all

$$
A \in \mathfrak{A}^{\mu}(M \backslash Y,(\gamma, \gamma-\mu))
$$

that are of the form

$$
A=A_{\text {sing }}+A_{\text {reg }}+C
$$

where $C \in \mathfrak{A}^{-\infty}(M, \boldsymbol{g})$, moreover, $A_{\text {reg }}, A_{\text {sing }} \in \mathfrak{A}^{\mu}(M \backslash Y,(\gamma, \gamma-\mu))$, and $A_{\text {reg }}$ vanishes in some neighbourhood $U$ of $Y$ (in the sense that $\left.A_{\text {reg }}\right|_{U}$ : $C_{0}^{\infty}(U \backslash Y) \rightarrow C^{\infty}(U \backslash Y)$ is the zero operator $)$. The operator $A_{\text {sing vanishes }}$ outside some other neighbourhood of $Y$ (in a similar sense), and $A_{\text {sing }}$ for $\operatorname{dim} Y=q_{k}>0$ is equal (modulo pull backs to $M$ ) a locally finite sum of operators of the form

$$
\varphi \mathrm{Op}_{z}(a+m+\sigma g \tilde{\sigma}) \psi
$$

referring to the local description of $M$ near $Y$ by wedges $X^{\Delta} \times \Omega, X \in \mathfrak{M}_{k-1}$, $\Omega \subseteq \mathbb{R}^{q_{k}}$ open $\left(q_{k}=\operatorname{dim} Y\right)$, for arbitrary (operator-valued) symbols $a, m, g$ of the above-mentioned kind, and functions $\varphi, \psi \in C_{0}^{\infty}(\Omega)$.

For $\operatorname{dim} Y=0, Y=\{v\}$, and $M$ locally near $v$ modelled on $X^{\Delta}$ for some $X \in \mathfrak{M}_{k-1}$, we set

$$
A_{\text {sing }}=t^{-\mu} \omega \operatorname{op}_{M}^{\delta-\frac{n}{2}}(h) \tilde{\omega}+M
$$


for some $h(t, w) \in C^{\infty}\left(\overline{\mathbb{R}}_{+}, \mathfrak{A}^{\mu}(X,(\gamma, \gamma-\mu) ; \mathbb{C})\right.$ and a linear combination $M$ of operators of the form

$$
m:=t^{-\mu+j} \omega \operatorname{op}_{M}^{\delta_{j}-\frac{n}{2}}\left(f_{j}\right) \tilde{\omega},
$$

$j \in \mathbb{N}, f_{j} \in \mathfrak{A}^{-\infty}\left(X,(\gamma, \gamma-\mu) ; \mathbb{C} \backslash V_{j}\right)$ for certain continuous asymptotic types $V_{j}$ (where we only have one summand for $j=0$ ), $\delta>\delta_{j}>\delta-j$, $V_{j} \cap \Gamma_{\frac{n+1}{2}-\delta_{j}}=\emptyset$ for all $j, n:=\operatorname{dim} X$.

By $\mathfrak{A}_{M+G}^{\mu}(M, \boldsymbol{g})$ for $\operatorname{dim} Y>0$ and $\mathfrak{A}_{M+G}(M, \boldsymbol{g})$ for $\operatorname{dim} Y=0$ we denote the subspace of all operators $K \in \mathfrak{A}^{\mu}(M, \boldsymbol{g})$ such that $\left.K\right|_{M \backslash Y} \in \mathfrak{A}^{-\infty}(M \backslash$ $Y,(\gamma, \gamma-\mu)$ ) for $k \geq 2$ (and $\left.K\right|_{M \backslash Y} \in L^{-\infty}(M \backslash Y)$ for $k=1$ ) such that

$$
K=K_{0}+C
$$

for some $C \in \mathfrak{A}^{-\infty}(M, \boldsymbol{g})$, and where $K_{0}$ is locally of the form (60) for where $a \equiv 0$. Moreover, by $\mathfrak{A}_{G}^{\mu}(M, \boldsymbol{g})$ we denote the subspace of such operators $K$ where in (60) we also have $m \equiv 0$.

The principal symbolic hierarchy. By (IA) for any operator in $\mathfrak{A}^{\mu}(M \backslash Y,(\gamma, \gamma-\mu)), M \in \mathfrak{M}_{k}$, with $Y \subset M$ being the minimal stratum, we have the principal symbolic hierarchy $\left(\sigma_{0}, \ldots, \sigma_{k-1}\right)$. For $A \in$ $\mathfrak{A}^{\mu}(M,((\gamma, \delta),(\gamma-\mu, \delta-\mu)))$ we now form

$$
\sigma(A)=\left(\sigma_{0}\left(\left.A\right|_{M \backslash Y}\right), \ldots, \sigma_{k-1}\left(\left.A\right|_{M \backslash Y}\right), \sigma_{k}(A)\right)
$$

using that $\left.A\right|_{M \backslash Y}$ belongs to $\mathfrak{A}^{\mu}(M \backslash Y,(\gamma, \gamma-\mu))$, such that $\sigma_{0}, \ldots, \sigma_{k-1}$ are known, and define $\sigma_{k}(A)$ as follows.

For $\operatorname{dim} Y=0$ and $X \in \mathfrak{M}_{k-1}$ compact we set

$$
\sigma_{k}(A)(w):=h(0, w)+f_{0}(w),
$$

where $h(0, w)$ are and $f_{0}(w)$ defined by (61) and (62), respectively. (63) is called the principal (corner) conormal symbol of $A$.

The symbolic component (63) represents a family of continuous operators

$$
\sigma_{k}(A)(w): H^{s, \gamma}(X) \rightarrow H^{s-\mu, \gamma-\mu}(X)
$$

$s \in \mathbb{R}$, parametrised by $w \in \Gamma_{\frac{n+1}{2}-\delta}, n=\operatorname{dim} X$.

For $\operatorname{dim} Y>0$ and $X \in \mathfrak{M}_{k-1}{ }^{2}$ compact we set

$$
\begin{aligned}
\sigma_{k}(A)(z, \zeta):= & t^{-\mu}\left\{\omega(t|\zeta|) \operatorname{op}_{M}^{\delta-\frac{\operatorname{dim} X}{2}}\left(h_{0}\right)(z, \zeta) \tilde{\omega}\left(t^{\prime}|\zeta|\right)+(1-\omega(t|\zeta|)\right. \\
& \left.\omega_{0}\left(t|\zeta|, t^{\prime}|\zeta|\right) \operatorname{Op}_{t}\left(p_{0}\right)(z, \zeta)\left(1-\tilde{\tilde{\omega}}\left(t^{\prime}|\zeta|\right)\right)\right\}+\sigma_{k}(m+g)(z, \zeta),
\end{aligned}
$$


where $\sigma_{k}(m+g)(z, \zeta)$ is simply the homogeneous principal symbol of $m+g$ in the sense of classical symbols with twisted homogeneity of order $\mu$. The symbol $\sigma_{k}(A)(z, \zeta)$ is defined for $(z, \zeta) \in \Omega \times\left(\mathbb{R}^{q_{k}} \backslash\{0\}\right)$.

It follows a family of operators

$$
\sigma_{k}(A)(z, \zeta): K^{s,(\gamma, \delta)}\left(X^{\Delta}\right) \rightarrow K^{s-\mu,(\gamma-\mu, \delta-\mu)}\left(X^{\Delta}\right)
$$

for all $s \in \mathbb{R}$, with the homogeneity property

$$
\sigma_{k}(A)(z, \lambda \zeta)=\lambda^{\mu} \kappa_{\lambda} \sigma_{k}(A)(z, \zeta) \kappa_{\lambda}^{-1}
$$

for all $\lambda \in \mathbb{R}_{+},\left(\kappa_{\lambda} u\right)(t, \cdot):=\lambda^{\frac{1+\operatorname{dim} X}{2}+(s-\gamma)} u(\lambda t, \cdot), \lambda \in \mathbb{R}_{+}$. We call $(65)$ the principal edge symbol of $A$ (where the minimal stratum of $M$ has the meaning of an edge).

Compositions. Let $M \in \mathfrak{M}_{k}$ be compact, and let $A \in \mathfrak{A}^{\mu}(M,(\gamma-$ $\nu, \gamma-(\mu+\nu)), B \in \mathfrak{A}^{\nu}(M,(\gamma, \gamma-\nu))$. Then we have $A B \in \mathfrak{A}^{\mu+\nu}(M,(\gamma, \gamma-$ $(\mu+\nu))$ and $\sigma(A B)=\sigma(A) \sigma(B)$ with componentwise multiplication of symbols (in the case $\operatorname{dim} Y=0$ that means for the $k$-th component $\sigma_{k}(A B)=$ $\left(T^{\nu} \sigma_{k}(A)\right) \sigma_{k}(B),\left(T^{\beta} f\right)(w)=f(w+\beta)$.

If $M$ is not compact we have a similar result when $A$ or $B$ is properly supported (the latter notion is a simple generalisation of the one in standard pseudo-differential operators on an open manifold (see Section 2.1), and every $A$ can be represented by a properly supported operator modulo an operator in $\mathfrak{A}^{-\infty}$.

The proof of the composition result follows by (IA) on the level of operators restricted to $M \backslash Y$ which already gives us the symbolic rule for the components $\sigma_{0}, \ldots, \sigma_{k-1}$. Then for the compositions of the operators near $Y$ we can apply similar arguments as in [8] (see also [48]) which shows the composition rule for $\sigma_{k}$.

Another useful aspect is that formal adjoints can be carried out within the calculus, with a corresponding rule on the level of symbols.

Similar relations hold for the parameter-dependent variants of the operator spaces.

\subsection{Ellipticity and an analogue of the Atiyah-Bott ob- struction}

The notion of ellipticity of an operator $A$ on a manifold $M \in \mathfrak{M}_{k}, k \geq$ 2 , can be defined in an iterative way when we assume by (IA) that the corresponding notion on any $N \in \mathfrak{M}_{k-1}$ is already introduced. Since the symbols are operator-valued, except for the interior symbol (i.e., the zeroth 
component on the maximal stratum) this is connected with the involved weighted spaces, also defined in an iterative manner (see Section 3.3 below). On a (for simplicity) compact $M \in \mathfrak{M}_{0}$, we take the standard Sobolev spaces $H^{s}(M)$ of smoothness $s \in \mathbb{R}$. On a compact $M \in \mathfrak{M}_{1}$ we have the weighted spaces $H^{s, \gamma}(M)$ ('cone-spaces' for $\operatorname{dim} Y=0$, 'edge-spaces' for $\operatorname{dim} Y>0$, according to the dimension of the minimal stratum) of smoothness $s \in \mathbb{R}$ and weight $\gamma \in \mathbb{R}$, and there are also subspaces $H_{P}^{s, \gamma}(M)$ with asymptotics of type $P$ (constant discrete, or continuous, and associated with weight data $(\gamma, \Theta)$ as well as with a fixed system of singular charts near $Y)$.

Definition 3.1 An operator $A \in \mathfrak{A}^{\mu}(M, \boldsymbol{g})$ for $\boldsymbol{g}=((\gamma, \delta),(\gamma-\mu, \delta-\mu))$ is called $\left(\sigma_{0}, \ldots, \sigma_{k-1}\right)$-elliptic, if $\left.A\right|_{M \backslash Y} \in \mathfrak{A}^{\mu}(M \backslash Y,(\gamma, \gamma-\mu))$ is elliptic with respect to all symbolic components $\sigma_{j}\left(\left.A\right|_{M \backslash Y}\right), j=0, \ldots, k-1$.

By (IA) we know what that means. For $k=0$ we have nothing other than the standard ellipticity, i.e., non-vanishing of the homogeneous principal symbol $\sigma_{0}(A)$ on $T^{*} M \backslash 0$ (or bijectivity of $\pi^{*} E \rightarrow \pi^{*} F, \pi: T^{*} M \backslash 0 \rightarrow M$ when $A$ acts between distributional sections of bundles $E, F \in \operatorname{Vect}(M))$.

For $k \geq 1$ the ellipticity condition for $\sigma_{0}(A)$ is given in Definition 1.7.

For $k=1$ and $\operatorname{dim} Y=0$, when $Y$ (for simplicity) consists of one conical point $v$, the component $\sigma_{1}(A)$ is the conormal symbol

$$
\sigma_{1}(A)(w): H^{s}(X) \rightarrow H^{s-\mu}(X)
$$

with $X \in \mathfrak{M}_{0}$ being the base of the cone near $v$, and the ellipticity with respect to $\gamma$ is defined to be the bijectivity of (66) for all $w \in \Gamma_{\frac{n+1}{2}-\gamma}$, $n=\operatorname{dim} X$ (because of the notation for general singularity orders we replace the former $\sigma_{\mathrm{c}}$ by $\sigma_{1}$; in a similar manner we proceed with $\sigma_{\wedge}$ ).

For $k=1$ and $\operatorname{dim} Y=q>0$ the component $\sigma_{1}(A)$ is the principal edge symbol

$$
\sigma_{1}(A)(y, \eta): K^{s, \gamma}\left(X^{\Delta}\right) \rightarrow K^{s-\mu, \gamma-\mu}\left(X^{\Delta}\right),
$$

parametrised by $(y, \eta) \in T^{*} Y \backslash 0$.

Theorem 3.2 Let $M \in \mathfrak{M}_{1}, \operatorname{dim} Y>0$, and let $A \in \mathfrak{A}^{\mu}(M,(\gamma, \gamma-\mu))$ be $\sigma_{0}$-elliptic. Then (67) is exit-elliptic on the infinite cone for $r \rightarrow \infty$ (of order 0 at infinity), for every $(y, \eta) \in T^{*} Y \backslash 0$ (see Definition 2.18).

Pointwise the operators in (67) belong to the cone algebra on $X^{\Delta}$, i.e., to $\mathfrak{A}^{\mu}\left(X^{\Delta},(\gamma, \gamma-\mu)\right)$; as such they have a principal conormal symbol

$$
\sigma_{1}\left(\sigma_{1}(A)\right)(y, w): H^{s}(X) \rightarrow H^{s-\mu}(X)
$$

with obvious meaning of notation (it is independent of $\eta$, since $\sigma_{1}$ in the cone case is computed by freezing $r$ at 0 in all arguments). 
Definition 3.3 Let $M \in \mathfrak{M}_{1}, A \in \mathfrak{A}^{\mu}(M,(\gamma, \gamma-\mu))$, and let $A$ be $\sigma_{0}$ elliptic. We then call $\sigma_{1}(A)(y, \eta)$ elliptic with respect to $\gamma$, if $(68)$ is a family of isomorphisms for all $y \in Y, w \in \Gamma_{\frac{n+1}{2}-\gamma}$.

Theorem 3.4 Let $A \in \mathfrak{A}^{\mu}(M,(\gamma, \gamma-\mu))$ be $\sigma_{0}$-elliptic, and let $\sigma_{1}(A)(y, \eta)$ be elliptic with respect to $\gamma$. Then (67) is a family of Fredholm operators for every $(y, \eta) \in T^{*} Y \backslash 0$.

Remark 3.5 By virtue of $\sigma_{1}(A)(y, \lambda \eta)=\lambda^{\mu} \kappa_{\lambda} \sigma_{1}(A)(y, \eta) \kappa_{\lambda}^{-1}$ for all $\lambda \in \mathbb{R}_{+}$, the Fredholm family (67) is completely determined by its restriction to $S^{*} Y$, the unit cosphere bundle in $T^{*} Y \backslash 0$ (a Riemannian metric on $Y$ is assumed to be fixed). Thus we obtain an index element

$$
\operatorname{ind}_{S^{*} Y} \sigma_{1}(A) \in K\left(S^{*} Y\right),
$$

where $K(\cdot)$ is the $K$-group (of corresponding classes of pairs $(E, F) \in \operatorname{Vect}(\cdot) \times$ Vect $(\cdot))$ on the compact space in parentheses, see Atiyah [4].

Bundle pull back with respect to the canonical projection $\pi: S^{*} Y \rightarrow Y$ gives rise to a homorphism $\pi^{*}: K(Y) \rightarrow K\left(S^{*} Y\right)$.

From boundary value problems, say, with the transmission property at the boundary, it is known that a necessary and sufficient condition for the existence of $J_{ \pm} \in \operatorname{Vect}(Y)$ such that $\sigma_{\partial}(A)(y, \eta)$ can be completed to a family of isomorphisms

$$
\sigma_{\partial}(\mathcal{A})(y, \eta): H^{s}\left(\mathbb{R}_{+}\right) \oplus J_{-, y} \rightarrow H^{s-\mu}\left(\mathbb{R}_{+}\right) \oplus J_{+, y},
$$

$(y, \eta) \in S^{*} Y$, is that

$$
\operatorname{ind}_{S^{*} Y} \sigma_{\partial}(A) \in \pi^{*} K(Y)
$$

see [5] for the case of elliptic differential operators, [6] for elliptic pseudodifferential operators with the transmission property. The extra entries in (69) (apart from the given upper left corner) represent the symbols of the additional operators in (28), and we then have a Fredholm operator

$$
\mathcal{A}: H^{s}(\operatorname{int} M) \oplus H^{s}\left(Y, J_{-}\right) \rightarrow H^{s-\mu}(\operatorname{int} M) \oplus H^{s-\mu}\left(Y, J_{+}\right),
$$

( $M$ in this case is a compact $C^{\infty}$ manifold with boundary $Y$, and $\operatorname{int} M=$ $M \backslash Y$ the interior).

A similar criterion for the existence of additional elliptic edge conditions for the edge-algebra has been given in [72], namely,

$$
\operatorname{ind}_{S^{*} Y} \sigma_{1}(A) \in \pi^{*} K(Y) \text {. }
$$

This admits the construction of an elliptic 'edge problem' of the form $(31)$. 
Remark 3.6 In such a process there arise two natural questions.

(i) What can be done when (70) or (72) is not satisfied?

(ii) If (70) or (72) hold, is there a way to describe ellipticity with respect to $\sigma_{1}(A)$ (in order to obtain Fredholm operators (71) or (31)) by avoiding extra entries of trace and potential type (possibly on the expense of additional operators in the upper left corner which are smooth over the main stratum)?

In the case (i) (which happens in boundary value problems, for instance, for Dirac operators in even dimensions) there is a general concept of posing so-called global projection conditions, as generalisations of the well-known APS- (or global spectral) conditions, see [87], [84]. The pseudo-differential algebra scenario for such conditions in boundary value problems is developed in [76] (see also [79], als well the case without the transmission property [82]), and in edge problems in [83]). In the case that (70) or (72) are satisfied, we can construct boundary and edge conditions, respectively. For boundary value problems with the transmission property those conditions in terms of additional entries cannot be avoided. However, if the transmission property is not satisfied, or in the general edge case, we have more freedom in the respective edge calculus which allows us to place the additional information in the upper left corner, according to the following result.

Theorem 3.7 Let $M \in \mathfrak{M}_{1}, \operatorname{dim} Y>0$, let $A \in \mathfrak{A}^{\mu}(M,(\gamma, \gamma-\mu))$ be $\sigma_{0}$-elliptic, and assume that $\sigma_{1}(A)$ is elliptic with respect to a weight $\gamma$ (cf. Definition 1.21 and Definition 3.3). Moreover, let the condition (72) be satisfied. Then there exists an operator $M+G \in \mathfrak{A}_{M+G}^{\mu}(M,(\gamma, \gamma-\mu))$ such that

$$
\sigma_{1}(A+M+G)(y, \eta): K^{s, \gamma}\left(X^{\Delta}\right) \rightarrow K^{s-\mu, \gamma-\mu}\left(X^{\Delta}\right)
$$

is a family of isomorphisms for all $s \in \mathbb{R}$.

Recall that for $k=1$ we have $\left.\mathfrak{A}_{M+G}^{\mu}(M,(\gamma, \gamma-\mu))\right|_{M \backslash Y} \subset L^{-\infty}(M \backslash Y)$; therefore, a smoothing but (in general) non-compact $M+G$-contribution to $A$ suffices to guarantee an analogue of the Shapiro-Lopatinskij condition without additional trace or potential operators.

For the abstract calculus of elliptic operators on $M$ for $M \in \mathfrak{M}_{k-1}, k \geq$ 2 , it seems to be advisable also to make such a manipulation, in order to suppress all the trace and potential entries on lower-dimensional strata, and to only explicitly take them into account in the last step, namely, with respect to the minimal stratum when the order of singularity is equal to $k$. Those can be again modified by such an $(M+G)$-trick, in order to prepare the 
same thing for $k+1$. The tool which is responsible for that is an analogue of Theorem 3.7 for any higher singularity order $>1$, see the $M+G$-notation in the preceding section under the headline 'Global corner operators of k-th generation'.

Remark 3.8 There are different variants of such ' $M+G$-theorems', also for the case $\operatorname{dim} Y=0$, or for parameter-dependent operators. For instance, let $\operatorname{dim} Y=0, M \in \mathfrak{M}_{k}, k \geq 2$, locally near $Y=\{v\}$ modelled on $X^{\Delta}$ for an $X \in \mathfrak{M}_{k-1}$, and let $A \in \mathfrak{A}^{\mu}(M, \boldsymbol{g})$ for $\boldsymbol{g}=((\gamma, \delta),(\gamma-\mu, \delta-\mu))$ be $\left(\sigma_{0}, \ldots, \sigma_{k-1}\right)$-elliptic, and in addition

$$
\sigma_{k}(A)(w): H^{s, \gamma}(X) \rightarrow H^{s-\mu, \gamma-\mu}(X)
$$

a family of isomorphisms, parametrised by $w \in \Gamma_{\frac{1+\operatorname{dim} X}{2}-\delta}$ (by definition this is just the ellipticity of $A$ for $\operatorname{dim} Y=0)$. Then for every $m \in \mathbb{Z}$ there exists an $M+G \in \mathfrak{A}_{M+G}^{\mu}(M,(\gamma, \gamma-\mu))$ (which is in this case only a 'smoothing' Mellin plus Green operator with respect to a corner point) such that $A+M+G$ is Fredholm and of index $m$. For $m \leq 0(m \geq 0)$ we can find $M+G$ in such a way that $A+M+G$ is injective (surjective); in particular, it is an isomorphism for $m=0$ ). Concerning constructions of this character in the case of boundary value problems, see [81], and of edge problems, [42].

Proposition 3.9 Let $M \in \mathfrak{M}_{k}, k \geq 1, Y$ its minimal stratum, $Y$ compact, $\operatorname{dim} Y>0, A \in \mathfrak{A}^{\mu}(M, \boldsymbol{g}), \boldsymbol{g}=((\gamma, \delta),(\gamma-\mu, \delta-\mu))$ (where the component $(\gamma, \gamma-\mu)$ disappears for $k=1)$; moreover, let $A$ be $\left(\sigma_{0}, \ldots, \sigma_{k-1}\right)$-elliptic (see Definition 3.1), and assume that

$$
\sigma_{k}(A)(y, \eta): K^{s,(\gamma, \delta)}\left(X^{\Delta}\right) \rightarrow K^{s-\mu,(\gamma-\mu, \delta-\mu)}\left(X^{\Delta}\right)
$$

is a family of Fredholm operators for $(y, \eta) \in T^{*} Y \backslash 0$. Assume that the analogue of the Atiyah-Bott obstruction vanishes, i.e.,

$$
\operatorname{ind}_{S^{*} Y} \sigma_{k}(A) \in \pi^{*} K(Y)
$$

$\pi: S^{*} Y \rightarrow Y$ the canonical projection (see the formula $(72)$ for $k=1$ ). Then there exist bundles $J_{ \pm} \in \operatorname{Vect}(Y)$ and $a 2 \times 2$ block matrix family of isomorphisms

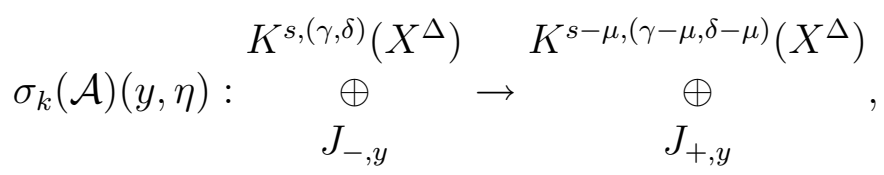

$(y, \eta) \in T^{*} Y \backslash 0$, where, when we write $\mathcal{A}=\left(\mathcal{A}_{i j}\right)_{i, j=1,2}$, the potential entry $\mathcal{A}_{12}$ maps into a subspace with $s=\infty$ and with asymptotics near the tip of the cone, and decrease of Schwartz type at infinity. 
Clearly we then have

$$
\operatorname{ind}_{S^{*} Y} \sigma_{k}(A)=\left[\pi^{*} J_{+}\right]-\left[\pi^{*} J_{-}\right]
$$

The entries of (73) are obtained by first constructing them on the compact space $S^{*} Y$ and then for arbitrary $\eta \neq 0$ via twisted homogeneity. For instance,

$$
\sigma_{k}\left(\mathcal{A}_{12}\right)(y, \lambda \eta)=\lambda^{\mu} \kappa_{\lambda} \sigma_{k}\left(\mathcal{A}_{12}\right)(y, \eta), \sigma_{k}\left(\mathcal{A}_{21}\right)(y, \lambda \eta)=\lambda^{\mu} \sigma_{k}\left(\mathcal{A}_{21}\right)(y, \eta) \kappa_{\lambda}^{-1}
$$

for all $\lambda \in \mathbb{R}_{+}$, with $\left\{\kappa_{\lambda}\right\}_{\lambda \in \mathbb{R}_{+}}$being the group action on the spaces over $X^{\Delta}$.

By multiplying the $i j$-th entries for $i+j>2$ by an excision function $\chi(\eta)$, we obtain locally on $Y$ symbols in

$$
\begin{gathered}
S_{\mathrm{cl}}^{\mu}\left(\Omega \times \mathbb{R}^{q} ; \mathbb{C}^{j_{-}}, K^{s-\mu,(\gamma-\mu, \delta-\mu)}\left(X^{\Delta}\right)\right), S_{\mathrm{cl}}^{\mu}\left(\Omega \times \mathbb{R}^{q} ; K^{s,(\gamma, \delta)}\left(X^{\Delta}\right), \mathbb{C}^{j_{+}}\right), \\
S_{\mathrm{cl}}^{\mu}\left(\Omega \times \mathbb{R}^{q} ; \mathbb{C}^{j_{-}}, \mathbb{C}^{j_{+}}\right) .
\end{gathered}
$$

They allow us to pass to associated $2 \times 2$ block matrix operators $\mathcal{A}$,

$$
\mathcal{A}: \underset{H^{s}\left(Y, J_{-}\right)}{H^{s,(\gamma, \delta)}(M)} \rightarrow \begin{gathered}
H^{s-\mu,(\gamma-\mu, \delta-\mu)}(M) \\
H^{s-\mu}\left(Y, J_{+}\right)
\end{gathered} .
$$

We then set

$$
\sigma(\mathcal{A})=\left(\sigma_{0}(A), \ldots, \sigma_{k-1}(A), \sigma_{k}(\mathcal{A})\right)
$$

where $\sigma_{k}(\mathcal{A})$ is just defined as the operator family (73). These constructions motivate an extension of $\mathfrak{A}^{\mu}(M, \boldsymbol{g})$ for $\left.\boldsymbol{g}=((\gamma, \delta), \gamma-\mu, \delta-\mu)\right)$ to a space $\mathfrak{A}^{\mu}(M, \boldsymbol{g}, \boldsymbol{v})$ of $2 \times 2$ matrices of operators $\mathcal{A}$ with a pair $\boldsymbol{v}:=\left(J_{-}, J_{+}\right)$ of vector bundles over the minimal stratum $Y$ of $M$. The nature of local symbols of the additional entries is already explained, namely, through (74), (75). There are also global smoothing operators in that space; the simple explanation will be omitted here. Moreover, we also have analogous operator spaces $\mathfrak{A}^{\mu}\left(M, \boldsymbol{g}, \boldsymbol{v} ; \mathbb{R}^{l}\right)$ with parameters.

Definition 3.10 An operator $\mathcal{A} \in \mathfrak{A}^{\mu}(M, \boldsymbol{g}, \boldsymbol{v})$ on $M \in \mathfrak{M}_{k}$ with minimal stratum $Y, \operatorname{dim} Y>0$, is called elliptic, if $A:=\mathcal{A}_{11}$ is $\left(\sigma_{0}, \ldots, \sigma_{k-1}\right)$-elliptic (see Definition 3.1) and if $\sigma_{k}(\mathcal{A})$ defines a family of isomorphisms (73) (the latter condition is an analogue of the Shapiro-Lopatinskij condition from the calculus of boundary value problems). For $\operatorname{dim} Y=0$ we gave the notion of ellipticity already in Remark 3.8.

In a similar manner we define parameter-dependent ellipticity in $\mathfrak{A}^{\mu}\left(M, \boldsymbol{g}, \boldsymbol{v} ; \mathbb{R}^{l}\right)$. 
Theorem 3.11 Let $M \in \mathfrak{M}_{k}, Y$ its minimal stratum, $\boldsymbol{g}=((\gamma, \delta),(\gamma-$ $\mu, \delta-\mu)$ ), where $(\gamma, \gamma-\mu)$ disappears for $k=1$. We then write $\boldsymbol{g}^{-1}=$ $((\gamma-\mu, \delta-\mu),(\gamma, \delta))$.

(i) An elliptic operator $A \in \mathfrak{A}^{\mu}(M, \boldsymbol{g})$ for $\operatorname{dim} Y=0$ induces a Fredholm operator

$$
A: H^{s,(\gamma, \delta)}(M) \rightarrow H^{s-\mu,(\gamma-\mu, \delta-\mu)}(M)
$$

for every $s \in \mathbb{R}$, and $A$ has a parametrix $P \in \mathfrak{A}^{-\mu}\left(M, \boldsymbol{g}^{-1}\right)$.

(ii) An elliptic operator $\mathcal{A} \in \mathfrak{A}^{\mu}(M, \boldsymbol{g}, \boldsymbol{v})$ for $\operatorname{dim} Y>0 \boldsymbol{v}:=\left(J_{-}, J_{+}\right)$, induces a Fredholm operator

$$
\mathcal{A}: H^{s,(\gamma, \delta)}(M) \oplus H^{s}\left(Y, J_{-}\right) \rightarrow H^{s-\mu,(\gamma-\mu, \delta-\mu)}(M) \oplus H^{s-\mu}\left(Y, J_{+}\right)
$$

for every $s \in \mathbb{R}$, and $\mathcal{A}$ has a parametrix $\mathcal{P} \in \mathfrak{A}^{-\mu}\left(M, \boldsymbol{g}^{-1}, \boldsymbol{v}^{-1}\right), \boldsymbol{v}^{-1}:=$ $\left(J_{+}, J_{-}\right)$.

\subsection{Weighted spaces}

In the preceding sections we employed various kinds of weighted spaces on $X^{\Delta}, X \in \mathfrak{M}_{k-1}$ or $M \in \mathfrak{M}_{k}$, called $K^{s,(\gamma, \delta)}\left(X^{\Delta}\right)$ and $H^{s,(\gamma, \delta)}(M)$, respectively (in the second case we assume that $M$ is compact, otherwise we need corresponding 'comp' and 'loc' analogues). We will sketch here some of the main aspects of the construction, also in an inductive manner. For $k=1$ we have the spaces $\mathcal{H}^{s, \gamma}\left(X^{\Delta}\right), H_{\text {cone }}^{s}\left(X^{\Delta}\right)$, and $\mathcal{K}^{s, \gamma}\left(X^{\Delta}\right), K^{s, \gamma}\left(X^{\Delta}\right)$, see the notation in (4), (5) and Remark 1.6. In the following Theorems 3.12 and 3.13 the manifold $X$ is assumed to be closed and compact.

Theorem 3.12 (i) For every $s \in \mathbb{R}$ there exists a parameter-dependent elliptic element $R^{\mu}(\varrho) \in L_{\mathrm{cl}}^{\mu}(X ; \mathbb{R})$ such that

$$
R^{\mu}(\varrho): H^{s}(X) \rightarrow H^{s-\mu}(X)
$$

is an isomorphism for every $s \in \mathbb{R}$.

(ii) The space $\mathcal{H}^{s, \gamma}\left(X^{\Delta}\right)$ for $s, \gamma \in \mathbb{R}$ can equivalently be defined as the completion of $C_{0}^{\infty}\left(\mathbb{R}_{+} \times X\right)$ with respect to the norm

$$
\left\{\frac{1}{2 \pi i} \int_{\Gamma_{\frac{n+1}{2}-\gamma}}\left\|R^{s}(\operatorname{Im} w)(M u)(w)\right\|_{L^{2}(X)}^{2} d w\right\}^{1 / 2} ;
$$

here $R^{s}(\varrho)$ is as in (i), and $M$ is the Mellin transform applied to $u$ with respect to $r$ as an element in $C_{0}^{\infty}\left(\mathbb{R}_{+}, C^{\infty}(X)\right)$. 
In particular, we can set

$$
\mathcal{H}^{s, \gamma}\left(X^{\Delta}\right)=\operatorname{op}_{M}^{\gamma-\frac{n}{2}}\left(R^{-s}\right) \mathcal{H}^{0,0}\left(X^{\Delta}\right),
$$

where $\mathcal{H}^{0,0}\left(X^{\Delta}\right)=r^{-\frac{n}{2}} L^{2}\left(\mathbb{R}_{+} \times X\right)$, and $R^{-s}$ is regarded as a function on $\Gamma_{\frac{n+1}{2}-\gamma} \ni w, \varrho=\operatorname{Im} w$, with values in $L_{\mathrm{cl}}^{-s}(X)$.

Theorem 3.13 There exists a parameter-dependent elliptic $\tilde{p}^{\mu}(\tilde{\varrho}, \tilde{\eta}) \in L_{\mathrm{cl}}^{\mu}\left(X ; \mathbb{R}_{\tilde{\varrho}, \tilde{\eta}}^{1+q}\right)$ such that when we set $p^{\mu}(r, \varrho, \eta):=\tilde{p}^{\mu}([r] \varrho,[r] \eta)$ for a strictly positive function $[r] \in C^{\infty}(\mathbb{R})$ with $[r]=|r|$ for $|r|>C$ for a $C>0$, the operator

$$
[r]^{-\mu} \mathrm{Op}_{r}\left(p^{\mu}\right)(\eta): H^{s ; g}\left(X_{\asymp}\right) \rightarrow H^{s-\mu ; g}\left(X_{\asymp}\right)
$$

is an isomorphism for every $s, g \in \mathbb{R}$ and every fixed $\eta \neq 0$. In particular, we can set $H^{s ; g}\left(X_{\asymp}\right)=\langle r\rangle^{-g}[r]^{s} \mathrm{Op}\left(p^{-s}\right)(\eta)\left([r]^{-\frac{n}{2}} L^{2}(\mathbb{R} \times X)\right)$ for any $\eta \neq 0$.

Remark 3.14 Taking into account Remark 2.20 we can define $\mathcal{K}^{s, \gamma ; g}\left(X^{\Delta}\right)$ equivalently as

$$
\mathcal{K}^{s, \gamma ; g}\left(X^{\Delta}\right):=\left\{\omega u+(1-\omega) v: u \in \mathcal{H}^{s, \gamma}\left(X^{\Delta}\right), v \in H^{s ; g}\left(X_{\asymp}\right)\right\}
$$

for any cut-off function $\omega$ (with $1-\omega:=0$ on the negative half-axis).

Recall that Definition 1.30 gave us the $H^{s, \gamma}$-spaces on a manifold $M$ with edge and subspaces with asymptotics of type $P$.

For the higher calculus $k \geq 2$ we can generate the weighted spaces and subspaces with asymptotics in a similar manner, using theorems of the following type.

Theorem 3.15 Let $X \in \mathfrak{M}_{k-1}$ be compact, and let $\gamma \in \mathbb{R}^{k-1}$. For every $\mu \in$ $\mathbb{R}$ there exists a parameter-dependent elliptic element $R^{\mu}(\varrho) \in \mathfrak{A}^{\mu}(X,(\gamma, \gamma-$ $\mu) ; \mathbb{R})$ such that

$$
R^{\mu}(\varrho): H^{s, \gamma}(X) \rightarrow H^{s-\mu, \gamma-\mu}(X)
$$

is an isomorphism for every $s \in \mathbb{R}$.

By (IA) we already know the spaces $H^{s, \gamma}(X)$ for (compact) $X \in \mathfrak{M}_{k-1}$. Also the spaces $H^{s, \gamma ; g}\left(X_{\asymp}\right)$ for $s, g \in \mathbb{R}, \gamma \in \mathbb{R}^{k-1}$, are assumed to be constructed (in contrast to the case $X \in \mathfrak{M}_{0}$ we have here the weights $\gamma$ along the base $X$ of $X \asymp=\mathbb{R} \times X)$.

Theorem 3.16 There exists a parameter-dependent elliptic $\tilde{p}(\tilde{\varrho}, \tilde{\eta}) \in \mathfrak{A}^{\mu}(X,(\gamma, \gamma-$ $\left.\mu) ; \mathbb{R}_{\tilde{\varrho}, \tilde{\eta}}^{1+q}\right)$ such that, when we define $p(r, \varrho, \eta)$ similarly as in Theorem 3.13 , the operator

$$
[r]^{-\mu} \mathrm{Op}_{r}(p)(\eta): H^{s, \gamma ; g}\left(X_{\asymp}\right) \rightarrow H^{s-\mu, \gamma-\mu ; g}\left(X_{\asymp}\right)
$$

is an isomorphism for every $s, g \in \mathbb{R}$ and every fixed $\eta \neq 0$. 
Similarly as in Theorems 3.12, 3.13 we can define the spaces $\mathcal{H}^{s,(\gamma, \delta)}\left(X^{\Delta}\right)$ and $H^{s, \gamma ; g}\left(X_{\asymp}\right)$, knowing the spaces for $s=0, g=0$ as weighted $L_{2}$-spaces on $\mathbb{R}_{+} \times X$ and $\mathbb{R} \times X$, respectively. We then define

$$
\mathcal{K}^{s,(\gamma, \delta) ; g}\left(X^{\Delta}\right):=\left\{\omega u+(1-\omega) v ; u \in \mathcal{H}^{s,(\gamma, \delta)}\left(X^{\Delta}\right), v \in H^{s, \gamma ; g}\left(X_{\asymp}\right)\right\}
$$

for any cut-off function on the half-axis.

The scheme of the definition of $H^{s,(\gamma, \delta)}$-spaces on an $M \in \mathfrak{M}_{k}$ is similar to Definition 1.30 (i), now based on $K^{s,(\gamma, \delta)}\left(X^{\Delta}\right):=\mathcal{K}^{s,(\gamma, \delta) ; s-\delta}\left(X^{\Delta}\right)$ with the group action $u(r, x) \rightarrow \lambda^{\frac{1+\operatorname{dim} X}{2}+(s-\delta)} u(\lambda r, x), \lambda \in \mathbb{R}_{+}$. The notion of subspaces with (continuous) asymptotics of type $P$ for $r \rightarrow 0$ is completely analogous to the one for $k=1$. In an iterative manner we can also formulate asymptotics of a similar structure along the other occurring distancevariables.

\subsection{Concluding remarks}

The calculus of operators that we sketched here is motivated by different concrete tasks in partial differential equations, especially boundary value problems, occurring in models of the applied sciences or mathematical physics, and by the desire to foresee the qualitative behaviour of solutions, also in order to launch numerical processes. For instance, knowing solvability, regularity, and parametrices of 'standard' elliptic boundary value problems in a smooth domain in the framework of the pseudo-differential calculus with the transmission property at the boundary (say, of Dirichlet or Neumann problems for second order equations) (see [6] or [63]) we can ask what has to happen in a calculus that solves mixed or crack problems in a non-smooth domain, for instance, in a cube or another configuration with 'polyhedral' geometry. An example is the Zaremba problem, where we have a jump from Dirichlet to Neumann conditions along an interface on the boundary of codimension 1 (see [13], [27]). By crack problems we understand boundary value problems in a domain with a slit representing a crack (e.g., a removed hypersurface of codimension 1 which itself has a boundary, smooth or non-smooth) at which we pose boundary conditions from both sides.

It turns out that, if we follow in a consequent manner the way of observing all the symbolic structures that play a role in boundary value problems in the smooth case, we are automatically led to what we perform here in our operator calculus, with conormal, edge, etc., symbols. To be more precise, we then have to take into account also conormal, edge, etc. symbols taking values in operators with the transmission property at the boundary of the base of some model cone, see [31], [28] [38]. In this connection we see 
that parameter-dependent variants of the already achieved calculi play an enormous role. In the process of constructing higher operator algebras the parameters are interpreted as covariables along singular strata in the next generations of operators. Parameter-dependent theories have been elaborated in a very 'early' stage of the development in PDE, see the work of Agranovich and Vishik [1], or Seeley [86].

Another source of motivation for our calculus is the study of geometric operators on manifolds with singularities, connected with operator algebras with symbolic structures, index theory, Hodge theory, or spectral theory. Let us mention in this context the new monograph [56] and refer to the bibliography there. In the present exposition we focused on the 'analytic content' of operator algebras with symbolic hierarchies, contributed by the singularities, and on ellipticity. It is possible to study parabolicity in a similar framework, see [34], [35], [36], [37], although there are some 'unexpected' difficulties. Also hyperbolic problems should be studied in the context of singularities of the spatial configurations. The geometric and topological aspects are interesting as well, and there are many new challenges and open problems, especially in respect to the scenario with global projection conditions in non-smooth situations see Remark 3.6, or the papers [43], [44] in an analogous spirit. There are many other beautiful branches of research, for instance, around parameter-dependent theories and the structure of resolvents, see the papers [17], [18], [33].

It seems altogether that the analysis on manifolds with singularities (although well established in some aspects through the work of different schools during the past decades) is at a new beginning, with many deep and fascinating problems. 


\section{References}

[1] M.S. Agranovich and M.I. Vishik, Elliptic problems with parameter and parabolic problems of general type, Uspekhi Mat. Nauk 19, 3 (1964), 53-161.

[2] R. Airapetyan and I. Witt, Isometric properties of the Hankel transform in weighted Sobolev spaces, Preprint 97/14, Institut für Mathematik, Potsdam, 1997.

[3] B. Ammann, R. Lauter, and V. Nistor, Pseudodifferential operators on manifolds with a lie structure at infinity, Ann. Math. (to appear).

[4] M.F. Atiyah, K-theory, Harvard University, Cambridge Mass., 1965.

[5] M.F. Atiyah and R. Bott, The index problem for manifolds with boundary, Coll. Differential Analysis, Tata Institute Bombay, Oxford University Press, Oxford, 1964, pp. 175-186.

[6] L. Boutet de Monvel, Boundary problems for pseudo-differential operators, Acta Math. 126 (1971), 11-51.

[7] D. Calvo, C.-I. Martin, and B.-W. Schulze, Symbolic structures on corner manifolds, RIMS Conf. dedicated to L. Boutet de Monvel on "Microlocal Analysis and Asymptotic Analysis", Kyoto, August 2004, Keio University, Tokyo, 2005, pp. 22-35.

[8] D. Calvo and B.-W. Schulze, Operators on corner manifolds with exits to infinity, Journal of Diff. Equ. 19, 2 (2006), 147-192.

[9] H.O. Cordes, A global parametrix for pseudo-differential operators over $\mathbb{R}^{n}$, with applications, Reprint, SFB 72, Universität Bonn, 1976.

[10] S. Coriasco and B.-W. Schulze, Edge problems on configurations with model cones of different dimensions, Osaka J. Math. 43 (2006), 1-40.

[11] G. De Donno and B.-W. Schulze, Meromorphic symbolic structures for boundary value problems on manifolds with edges, Math. Nachr. 279, 4 (2006), 368-399.

[12] N. Dines, Elliptic operators on corner manifolds, Ph.D. thesis, University of Potsdam, 2006. 
[13] N. Dines, G. Harutjunjan, and B.-W. Schulze, Mixed boundary value problems and parametrices in the edge calculus, Bull. des Sciences Math. (to appear).

[14] Ch. Dorschfeldt, U. Grieme, and B.-W. Schulze, Pseudo-differential calculus in the Fourier-edge approach on non-compact manifolds, Advances in Partial Differential Equations (Spectral Theory, Microlocal Analysis, Singular Manifolds), Akademie Verlag, Berlin, 1997, pp. 249-299.

[15] M. Dreher and I. Witt, Edge Sobolev spaces and weakly hyperbolic operators, Ann. Mat. Pura Appl., 180 (2002), 451-482.

[16] Ju. V. Egorov and B.-W. Schulze, Pseudo-differential operators, singularities, applications, Operator Theory, Advances and Applications, vol. 93, Birkhäuser Verlag, Basel, 1997.

[17] J.B. Gil, T. Krainer, and G. Mendoza, Geometry and spectra of closed extensions of elliptic cone operators, Canadian J. Math., to appear.

[18] J.B. Gil, T. Krainer, and G. Mendoza, Resolvents of elliptic cone operators, J. Funct. Anal., to appear.

[19] J.B. Gil, B.-W. Schulze, and J. Seiler, Holomorphic operator-valued symbols for edge-degenerate pseudo-differential operators, Differential Equations, Asymptotic Analysis, and Mathematical Physics, Math. Res., vol. 100, Akademie Verlag Berlin, 1997, pp. 113-137.

[20] J.B. Gil, B.-W. Schulze, and J. Seiler, Cone pseudodifferential operators in the edge symbolic calculus, Osaka J. Math. 37 (2000), 219-258.

[21] I.C. Gohberg and E.I. Sigal, An operator generalization of the logarithmic residue theorem and the theorem of Rouché, Math. USSR Sbornik 13, 4 (1971), 603-625.

[22] B. Gramsch, Meromorphie in der Theorie von Fredholmoperatoren mit Anwendungen auf elliptische Differentialgleichungen, Math. Ann. 188 (1970), 97-112.

[23] B. Gramsch, Inversion von Fredholmfunktionen bei stetiger und holomorpher Abhängigkeit von Parametern, Math. Ann. 214 (1975), 95-147.

[24] B. Gramsch and W. Kaballo, Multiplicative decompositions of holomorphic Fredholm functions and $\Psi^{*}$-algebras, Math. Nachr. 204 (1999), 83100. 
[25] G. Grubb, Functional calculus of pseudo-differential boundary problems. Second Edition, Birkhäuser Verlag, Boston, 1996.

[26] G. Harutjunjan and B.-W. Schulze, The relative index for corner singularities, Integral Equations Operator Theory 54, 3 (2006), 385-426.

[27] G. Harutjunjan and B.-W. Schulze, The Zaremba problem with singular interfaces as a corner boundary value problem, Potential Analysis 25, 4 (2006), 327-369.

[28] G. Harutjunjan and B.-W. Schulze, Elliptic mixed, transmission and singular crack problems, European Mathematical Soc., Zürich, to appear.

[29] T. Hirschmann, Functional analysis in cone and edge Sobolev spaces, Ann. Global Anal. Geom. 8, 2 (1990), 167-192.

[30] I.L. Hwang, The $L^{2}$-boundness of pseudodifferential operators, Trans. Amer. Math. Soc. 302 (1987), 55-76.

[31] D. Kapanadze and B.-W. Schulze, Crack theory and edge singularities, Kluwer Academic Publ., Dordrecht, 2003.

[32] V.A. Kondratyev, Boundary value problems for elliptic equations in domains with conical points, Trudy Mosk. Mat. Obshch. 16 (1967), 209292.

[33] T. Krainer, Resolvents of elliptic boundary problems on conic manifolds, Comm. Part. Diff. Equ., to appear.

[34] T. Krainer, Parabolic pseudodifferential operators and long-time asymptotics of solutions, Ph.D. thesis, University of Potsdam, 2000.

[35] T. Krainer, The calculus of Volterra Mellin pseudodifferential operators with operator-valued symbols, Advances in Partial Differential Equations (Parabolicity, Volterra Calculus, and Conical Singularities) (S. Albeverio, M. Demuth, E. Schrohe, and B.-W. Schulze, eds.), Oper. Theory Adv. Appl., vol. 138, Birkhäuser Verlag, Basel, 2002, pp. 47-91.

[36] T. Krainer, Volterra families of pseudodifferential operators, Advances in Partial Differential Equations (Parabolicity, Volterra Calculus, and Conical Singularities) (S. Albeverio, M. Demuth, E. Schrohe, and B.-W. Schulze, eds.), Oper. Theory Adv. Appl., vol. 138, Birkhäuser Verlag, Basel, 2002, pp. 1-45. 
[37] T. Krainer, On the inverse of parabolic boundary value problems for large times, Japan. J. Math. 30, 1 (2004), 91-163.

[38] T. Krainer and B.-W. Schulze, On the inverse of parabolic systems of partial differential equations of general form in an infinite spacetime cylinder, Advances in Partial Differential Equations (Parabolicity, Volterra Calculus, and Conical Singularities) (S. Albeverio, M. Demuth, E. Schrohe, and B.-W. Schulze, eds.), Oper. Theory Adv. Appl., vol. 138, Birkhäuser Verlag, Basel, 2002, pp. 93-278.

[39] T. Krainer and B.-W. Schulze, The conormal symbolic structure of corner boundary value problems, Operator Theory: Advances and Applications, vol. 155, Birkhäuser Verlag, Basel, 2004, pp. 19-64.

[40] T. Krainer and B.-W. Schulze, Long-time asymptotics with geometric singularities in the spatial variables, Contemporary Mathematics 364 (2004), 103-126.

[41] H. Kumano-go, Pseudo-differential operators, The MIT Press, Cambridge, Massachusetts and London, England, 1981.

[42] X. Liu and B.-W. Schulze, Ellipticity on manifolds with edges and boundary, Monatshefte für Mathematik 146, 4 (2005), 295-331.

[43] P. Loya, Index theory of Dirac operators on manifolds with corners up to codimension two, Advances in Partial Differential Equations (Aspects of boundary problems in analysis and geometry) (J. Gil, Th. Krainer, and I. Witt, eds.), Oper. Theory Adv. Appl., Birkhäuser Verlag, Basel, 2004, pp. 131-169.

[44] P. Loya, The index of b-pseudodifferential operators on manifolds with corners, Ann. Global Anal. Geom. 27, 2 (2005), 105-113.

[45] L. Maniccia and B.-W. Schulze, An algebra of meromorphic corner symbols, Bull. des Sciences Math. 127, 1 (2003), 55-99.

[46] L. Maniccia, B.-W. Schulze, and I. Witt, Ellipticity on manifolds with edges and parameter-dependent calculus, Preprint, Institut für Mathematik, Potsdam, 2001, (in preparation).

[47] C.-I. Martin, Operator algebras and complexes of operators on manifolds with corners, Ph.D. thesis, University of Potsdam, 2006. 
[48] C.-I. Martin and B.-W. Schulze, The quantisation of edge symbols, Modern Trends in Pseudo-Differential Operators, Birkhäuser Verlag, Basel, 2006, to appear.

[49] R. Mazzeo, Elliptic theory of differential edge operators I, Comm. Partial Differential Equations 16 (1991), 1615-1664.

[50] R.B. Melrose, The Atiyah-Patodi-Singer index theorem, Research Notes in Mathematics, A.K. Peters, Wellesley, 1993.

[51] R.B. Melrose and G.A. Mendoza, Elliptic operators of totally characteristic type, Preprint MSRI 047 - 83, Math. Sci. Res. Institute, 1983.

[52] R.B. Melrose and Piazza P., Analytic K-theory on manifolds with corners, Adv. in Math. 92, 1 (1992), 1-26.

[53] V. Nazaikinskij, A. Savin, B.-W. Schulze, and B. Ju. Sternin, Elliptic theory on manifolds with nonisolated singularities: II. Products in elliptic theory on manifolds with edges, Preprint 2002/15, Institut für Mathematik, Potsdam, 2002.

[54] V. Nazaikinskij, A. Savin, B.-W. Schulze, and B. Ju. Sternin, Elliptic theory on manifolds with nonisolated singularities: III. The spectral flow of families of conormal symbols., Preprint 2002/20, Institut für Mathematik, Potsdam, 2002.

[55] V. Nazaikinskij, A. Savin, B.-W. Schulze, and B. Ju. Sternin, On the homotopy clssification of elliptic operators on manifolds with edges., Preprint 2004/16, Institut für Mathematik, Potsdam, 2004.

[56] V. Nazaikinskij, A. Savin, B.-W. Schulze, and B. Ju. Sternin, Elliptic theory on singular manifolds, Taylor \& Francis, London, New York, 2005 .

[57] V. Nazaikinskij, A. Savin, and B. Sternin, Elliptic theory on manifolds with corners: I. dual manifolds and pseudodifferential operators, arXiv: math. OA/0608353v1.

[58] V. Nazaikinskij, A. Savin, and B. Sternin, Elliptic theory on manifolds with corners: II. homotopy classification and K-homology, arXiv: math. $\mathrm{KT} / 0608354 \mathrm{v} 1$.

[59] V. Nazaikinskij, A. Savin, and B. Sternin, On the homotopy classification of elliptic operators on stratified manifolds, arXiv: math. $\mathrm{KT} / 0608332 \mathrm{v} 1$. 
[60] V. Nazaikinskij, A. Savin, and B. Sternin, Pseudodifferential operators on stratified manifolds, arXiv: math. AP/0512025v1.

[61] A. Oliaro and B.-W. Schulze, Parameter-dependent boundary value problems on manifolds with edges, Int. Journal of Pure and Applied Math. 13, 1 (2004), 39-100.

[62] C. Parenti, Operatori pseudo-differenziali in $\mathbb{R}^{n}$ e applicazioni, Annali Mat. Pura Appl. (4) 93 (1972), 359-389.

[63] S. Rempel and B.-W. Schulze, Index theory of elliptic boundary problems, Akademie-Verlag, Berlin, 1982.

[64] S. Rempel and B.-W. Schulze, Parametrices and boundary symbolic calculus for elliptic boundary problems without transmission property, Math. Nachr. 105 (1982), 45-149.

[65] S. Rempel and B.-W. Schulze, Branching of asymptotics for elliptic operators on manifolds with edges, Proc. "Partial Differential Equations" (Warsaw), Banach Center Publ., vol. 19, PWN Polish Scientific Publisher, 1984.

[66] S. Rempel and B.-W. Schulze, Mellin symbolic calculus and asymptotics for boundary value problems, Seminar Analysis 1984/1985 (1985), 23-72.

[67] S. Rempel and B.-W. Schulze, Complete Mellin and Green symbolic calculus in spaces with conormal asymptotics, Ann. Global Anal. Geom. 4, 2 (1986), 137-224.

[68] S. Rempel and B.-W. Schulze, Asymptotics for elliptic mixed boundary problems (pseudo-differential and Mellin operators in spaces with conormal singularity), Math. Res., vol. 50, Akademie-Verlag, Berlin, 1989.

[69] B.-W. Schulze, Ellipticity and continuous conormal asymptotics on manifolds with conical singularities, Math. Nachr. 136 (1988), 7-57.

[70] B.-W. Schulze, Corner Mellin operators and reduction of orders with parameters, Ann. Sc. Norm. Sup. Pisa Cl. Sci. 16, 1 (1989), 1-81.

[71] B.-W. Schulze, Pseudo-differential operators on manifolds with edges, Symp. "Partial Differential Equations", Holzhau 1988, Teubner-Texte zur Mathematik, vol. 112, Teubner, Leipzig, 1989, pp. 259-287.

[72] B.-W. Schulze, Pseudo-differential operators on manifolds with singularities, North-Holland, Amsterdam, 1991. 
[73] B.-W. Schulze, The Mellin pseudo-differential calculus on manifolds with corners, Symp. "Analysis in Domains and on Manifolds with Singularities", Breitenbrunn 1990, Teubner-Texte zur Mathematik, vol. 131, Teubner, Leipzig, 1992, pp. 208-289.

[74] B.-W. Schulze, Pseudo-differential boundary value problems, conical singularities, and asymptotics, Akademie Verlag, Berlin, 1994.

[75] B.-W. Schulze, Boundary value problems and singular pseudo-differential operators, J. Wiley, Chichester, 1998.

[76] B.-W. Schulze, An algebra of boundary value problems not requiring Shapiro-Lopatinskij conditions, J. Funct. Anal. 179 (2001), 374-408.

[77] B.-W. Schulze, Operator algebras with symbol hierarchies on manifolds with singularities, Advances in Partial Differential Equations (Approaches to Singular Analysis) (J. Gil, D. Grieser, and Lesch M., eds.), Oper. Theory Adv. Appl., Birkhäuser Verlag, Basel, 2001, pp. 167-207.

[78] B.-W. Schulze, Operators with symbol hierarchies and iterated asymptotics, Publications of RIMS, Kyoto University 38, 4 (2002), 735-802.

[79] B.-W. Schulze, Toeplitz operators, and ellipticity of boundary value problems with global projection conditions., Advances in Partial Differential Equations (Aspects of boundary problems in analysis and geometry) (J. Gil, Th. Krainer, and I. Witt, eds.), Oper. Theory Adv. Appl., Birkhäuser Verlag, Basel, 2004, pp. 342-429.

[80] B.-W. Schulze, The structure of operators on manifolds with polyhedral singularities, Preprint 2006/05, Institut für Mathematik, Potsdam, 2006, arXiv:math.AP/0610618, 2006.

[81] B.-W. Schulze and J. Seiler, The edge algebra structure of boundary value problems, Annals of Global Analysis and Geometry 22 (2002), 197-265.

[82] B.-W. Schulze and J. Seiler, Pseudodifferential boundary value problems with global projection conditions, J. Funct. Anal. 206, 2 (2004), 449-498.

[83] B.-W. Schulze and J. Seiler, Edge operators with conditions of Toeplitz type, J. of the Inst. Math. Jussieu. 5, 1 (2006), 101-123.

[84] B.-W. Schulze, B.Ju. Sternin, and V.E. Shatalov, On general boundary value problems for elliptic equations, Sbornik Mathematics 189, 10 (1998), 1573-1586. 
[85] B.-W. Schulze and A. Volpato, Green operators in the edge quantisation of elliptic operators, Preprint 2004/25, Institut für Mathematik, Potsdam, 2004.

[86] R. Seeley, Complex powers of an elliptic operator, Proc. Sympos. Pure Math., vol. 10, 1967, pp. 288-307.

[87] R. Seeley, Topics in pseudo-differential operators, C.I.M.E. Conference on pseudo-differential operators, Stresa 1968 (Cremonese, Roma), 1969, pp. 167-305.

[88] J. Seiler, Pseudodifferential calculus on manifolds with non-compact edges, Ph.D. thesis, University of Potsdam, 1998.

[89] J. Seiler, Continuity of edge and corner pseudo-differential operators, Math. Nachr. 205 (1999), 163-182.

[90] J. Seiler, The cone algebra and a kernel characterization of Green operators, Advances in Partial Differential Equations (Approaches to Singular Analysis) (J. Gil, D. Grieser, and Lesch M., eds.), Oper. Theory Adv. Appl., Birkhäuser, Basel, 2001, pp. 1-29.

[91] M.A. Shubin, Pseudodifferential operators in $\mathbb{R}^{n}$, Dokl. Akad. Nauk SSSR 196 (1971), 316-319.

[92] N. Teleman, Global analysis on PL-manifolds, Trans. A.M.S. 256 (1979), 49-88.

[93] N. Teleman, Combinatorial Hodge theory and signature operator, Invent. Math. 61 (1980), 227-249.

[94] F. Treves, Introduction to pseudo-differential and Fourier integral operators, vol. 1 and 2, Plenum, New York, 1985.

[95] I. Witt, On the factorization of meromorphic Mellin symbols, Advances in Partial Differential Equations (Parabolicity, Volterra Calculus, and Conical Singularities) (S. Albeverio, M. Demuth, E. Schrohe, and B.-W. Schulze, eds.), Oper. Theory Adv. Appl., vol. 138, Birkhäuser Verlag, Basel, 2002, pp. 279-306. 